\title{
Contributions to Topological Formulations for the Dynamic Simulation of Vehicles
}

\author{
YONGJUN PAN
}

\author{
A Thesis submitted in partial fulfillment for the \\ degree of Doctor of Philosophy in Mechanical Engineering \\ in the \\ Escuela Técnica Superior de Ingenieros Industriales \\ Universidad Politécnica de Madrid
}

Supervisors

Dr. Javier García de Jalón de la Fuente

Dr. Alfonso Callejo Goena

Madrid, 2016 


\begin{abstract}
There exist a wide variety of topological formulations that deal with the dynamic simulation of medium-large multibody systems. In recent decades, with the strong demand of real-time simulation of vehicle models, a great deal of interest has emerged around efficient topological formulations. This Thesis investigates the computational efficiency of different numerical approaches applied to a number of realistic vehicle models, and introduces a number of novel techniques into a state-of-the-art topological formulation.

First, three state-of-the-art topological formulations (generalized semi-recursive, double-step semi-recursive and subsystem synthesis) have been investigated in terms of underlying principles, numerical efficiency and accuracy. They have been numerically implemented and applied to a 28-degree-of-freedom, open-loop rover and a 16-degree-of-freedom, closed-loop sedan vehicle. Second, an original improvement to the rod-removal technique, based on the approximation of second-derivative-based inertia forces, has been introduced into the double-step semi-recursive formulation. This reduces the complexity of the system inertia matrix and improves computational efficiency. Three extrapolation methods are used to approximate the accelerations: constant, linear and quadratic Lagrange. Further, a novel method for the matrix partitioning of the rod inertia has been introduced to preserve accuracy. Third, an iterative refinement technique for the speedup of the 4th-order Runge-Kutta integrator has been presented, whereby the generalized mass matrix factorization and its calculation are avoided. A 16-degree-of-freedom sedan vehicle model and a 40-degree-of-freedom semi-trailer truck model have been simulated in detail to evaluate the computational efficiency and accuracy.

In summary, a number of improvements to both the form of the equations of motion and the time integration scheme have been implemented within a topological formulation. The improvements are backed by a comparison with alternative state-of-the-art methods and the simulation of realistic vehicles.
\end{abstract}




\section{Resumen}

Existen numerosos métodos para la simulación dinámica de sistemas multicuerpo de tamaño mediano-grande. En las últimas décadas, debido a la fuerte demanda de simulación de vehículos en tiempo real, se ha suscitado un gran interés en torno a las formulaciones topológicas eficientes. En esta Tesis se investiga la eficiencia computacional de varios métodos numéricos aplicados a modelos realistas de vehículos, y se introducen técnicas novedosas en una formulación topológica puntera.

En primer lugar, tres formulaciones topológicas de vanguardia (semirrecursiva generalizada, semirrecursiva de doble paso y síntesis de subsistemas) han sido investigadas en cuanto a sus principios básicos, eficiencia numérica y exactitud. Además, han sido numéricamente implementadas y aplicadas a un rover de 28 grados de libertad y cadena abierta, y un vehículo sedán de 16 grados de libertad y cadena cerrada. En segundo lugar, se ha introducido una mejora original en la técnica de eliminación de rods (barras biarticuladas) del método semirrecursivo de doble paso, basada en la aproximación de las fuerzas inerciales relacionadas con segundas derivadas. Esto reduce la complejidad de la matriz de masa y mejora la eficiencia computacional. Tres métodos han sido utilizados para extrapolar las aceleraciones: constante, lineal y Lagrange cuadrático. Además, se ha introducido un nuevo método para particionar la inercia de los rods y preservar la exactitud. En tercer lugar, una técnica basada en el refinamiento iterativo ha sido introducida para acelerar el integrador Runge-Kutta de cuarto orden, por la cual se evita la factorización de la matriz de masa y su cálculo. Un vehículo sedán de 16 grados de libertad y un camión de 40 grados de libertad han sido simulados en detalle para evaluar la eficiencia computacional y exactitud.

En resumen, se han implementado varias mejoras en una formulación topológica con respecto a la forma de las ecuaciones del movimiento y el método de integración numérica. Las mejoras han sido respaldadas con una comparación con formulaciones de última generación y con la simulación realista de vehículos. 


\section{Acknowledgements}

First and foremost, I am deeply grateful for the continuous support, insight and patience of my supervisors, Javier García de Jalón and Alfonso Callejo. Without their help and gentle prodding, this Thesis would not have been completed.

I would like to express my special appreciation to Javier García de Jalón. He taught me so much in the past four years, and introduced me to the field of multibody dynamics.

A very big thank you goes to my co-supervisor Alfonso Callejo for his detailed guidance and constant feedback, as well as József Kövecses; they both hosted my research stay at the Centre for Intelligent Machines (McGill University).

Thanks also to Francisco Aparicio, José María López, Francisco Javier Páez, Blanca Arenas, Roger Wehage, Andrés Francisco Hidalgo, Francisco Javier Funes, María Dolores Gutiérrez, Wilmar Cabrera, José Luis Bueno and José Luis Ricón for their help and collaboration, as well as the whole crew at the University Institute for Automobile Research (INSIA, Universidad Politécnica de Madrid).

I would also like to say a heartfelt thank you to my parents for always believing in me and encouraging me to follow my dreams, and to my friends, who always supported me.

Finally, I gratefully acknowledge the funding from the Chinese Scholarship Council (CSC) and the support from the Spanish Ministry of Science and Innovation via research projects. 


\section{Contents}

1 Introduction 1

1.1 Classical global formulations . . . . . . . . . . . . 3

1.1.1 Index-1 formulation . . . . . . . . . . . 3

1.1.2 Null space method .............. 5

1.2 State of the art of topological formulations . . . . . . . . . . 7

1.3 Double-step semi-recursive formulation . . . . . . . . . . . 8

1.3.1 Open-loop equations . . . . . . . . . . . . 8

1.3.2 Closed-loop equations . . . . . . . . . . . 11

1.4 Challenges and motivation . . . . . . . . . . . 13

1.5 Objectives and structure . . . . . . . . . . . . . . 14

2 Comparison of semi-recursive and subsystem synthesis algorithms 17

2.1 Introduction . . . . . . . . . . . . . . 17

2.2 Generalized semi-recursive formulation . . . . . . . . . 20

2.2 .1 Dynamic equations . . . . . . . . . . . . 21

2.2.2 Sets of bodies and derivatives . . . . . . . . . 26

2.3 Subsystem synthesis formulation . . . . . . . . . . . . . . 27

2.4 Results . . . . . . . . . . . . . . . . . . 31

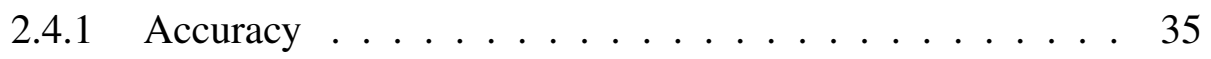

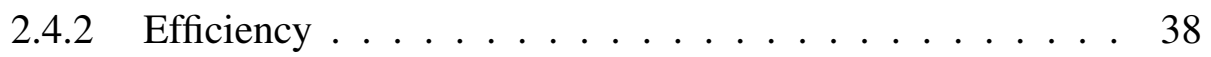

2.5 Discussion ......................... 42 
3 Efficient and accurate modeling of rigid rods 45

3.1 Introduction . . . . . . . . . . . . . . 46

3.2 Double-step semi-recursive vehicle dynamics . . . . . . . . . 50

3.3 Rod-removal technique . . . . . . . . . . . . . . . . 51

3.4 Approximation of rod-related SDIFs . . . . . . . . . . . . 56

3.5 Partitioning of rod mass matrix . . . . . . . . . . . 62

3.6 Results . . . . . . . . . . . . . . . 65

3.6 .1 Accuracy .................. 66

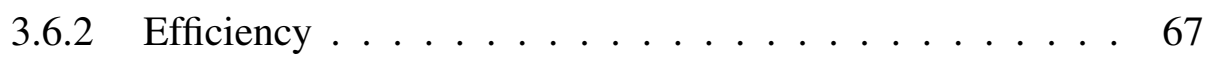

3.7 Discussion . . . . . . . . . . . . . . . 68

4 Iterative refinement of accelerations $\quad 71$

4.1 Introduction . . . . . . . . . . . . . . 71

4.2 Algorithm . . . . . . . . . . . . . . . . 73

4.2.1 Initial value determination . . . . . . . . . . . 74

4.2.2 Iterative refinement process . . . . . . . . . 76

4.2.3 Termination criteria . . . . . . . . . . . . 80

4.3 Results . . . . . . . . . . . . . . . 81

4.3.1 Accuracy .................. 83

4.3 .2 Efficiency . . . . . . . . . . . . 87

4.4 Discussion . . . . . . . . . . . . . . . . . . . . 89

5 Conclusions $\quad 91$

5.1 Contributions ......................... 91

5.2 Future work . . . . . . . . . . . . . . 92 


\section{Chapter 1}

\section{Introduction}

Multibody systems are constrained mechanical systems consisting of interconnected rigid or flexible bodies, each of which undergoes relative rotations or displacements and is subject to constraint forces resulting from the mechanical joints. Large relative rotations and displacements introduce geometric nonlinearities. Mechanical joints introduce algebraic constraints and lead to a set of governing equations combining differential and algebraic equations. Multibody systems are present in many fields of engineering such as automobiles, machinery, robotics, aerospace, biomechanics and so on. Although the equations of motion that govern the dynamic behavior have been investigated since the time of Newton, Euler and Lagrange, modern methods arose to solve medium-large spacecraft and robotics problems only about 50 years ago.

The first spacecraft and robotics applications were open-loop multibody systems [59, 62, 87, 88, 109]. Their equations of motion were formulated in absolute (reference-point) coordinates and then projected onto relative coordinates. The usage of relative coordinates often requires less storage and ensures higher computational efficiency. On the other hand, much attention has been paid to vehicle applications since the seventies $[34,77,82,103,113]$, which normally are closed-loop multibody systems. Commercial software packages that used highly 
constrained Cartesian coordinates, such as MSC.Adams and Dads, were developed. They are based on absolute coordinates and allow the direct and systematic solution of the equations of motion. They are also referred to as global formulations, in the sense that they consider both open- and closed-loop systems in exactly the same direct and general way. The computational efficiency of global formulations is relatively low. They are widely used in commercial software packages due to their simplicity and robustness.

Topological formulations were proposed to increase the efficiency by taking advantage of the system topology. The equations of motion were formulated in relative coordinates. Topological formulations are considered to be more efficient due to the implementation of recursive kinematics and dynamics and a reduced number of arithmetic operations.

Bae and Haug [17], Featherstone [36] and Anderson [2-4] developed several topological formulations for the direct and inverse dynamics of open-loop systems with $O(n)$ arithmetic operations. They are referred to as fully-recursive formulations because their equations of motion are fully-recursive without any loop-closure constraints. The usual way to deal with closed-loop systems is to open the closed loops by cutting certain joints. In this way, the fully-recursive formulations can be implemented. The equations of motion of closed-loop systems can be obtained by imposing the loop-closure constraint equations that result from the cut joints. Most authors [5, 8, 18, 44, 94] use the simpler semi-recursive formulations where a small set of constraint equations has to be solved after the implementation of the open-loop fully-recursive kinematics. Semi-recursive formulations arrive at equations of motion in terms of relative coordinates, which can be numerically integrated through explicit or implicit integrators in an efficient way.

Theoretical background relevant to this Thesis is presented in the following three Sections. In the first Section, the classical index-1 formulation stemming from Lagrange's equations of the first kind is introduced in terms of Cartesian coordinates. Popular null space method, subsequently, is presented as an alternative 
solution method. In the second Section, topological formulations are reviewed. In the third Section, the double-step semi-recursive formulation, based on which the Thesis was carried out, is introduced. Next, the challenges and motivation of this Thesis are analyzed in the fourth Section and the Thesis structure is described in the last Section.

\subsection{Classical global formulations}

\subsubsection{Index-1 formulation}

There are plenty of methods for the expression and solution of the dynamic equations that govern constrained multibody systems. The most widely used ones stem from the Lagrange's equations of the first kind. In the descriptor form, using Cartesian coordinates, the differential equations of motion take the form:

$$
\mathbf{M}(\mathbf{q}) \ddot{\mathbf{q}}+\boldsymbol{\Phi}_{\mathbf{q}}^{\mathrm{T}}(\mathbf{q}, t) \boldsymbol{\lambda}=\mathbf{Q}(\mathbf{q}, \dot{\mathbf{q}}, t)
$$

where $\mathbf{q} \in \mathbb{R}^{n}$ is the array of Cartesian coordinates that represent the system positions; $\mathbf{M}(\mathbf{q}) \in \mathbb{R}^{n \times n}$ is the mass matrix; $\mathbf{Q}(\mathbf{q}, \dot{\mathbf{q}}, t) \in \mathbb{R}^{n}$ is the array of external and velocity-dependent inertial forces; $\boldsymbol{\lambda} \in \mathbb{R}^{m}$ contains the Lagrange multipliers associated with the constraints. Assuming that all constraints are holonomic, matrix $\boldsymbol{\Phi}_{\mathbf{q}} \in \mathbb{R}^{m \times n}$ is called the constraint Jacobian matrix. The position, velocity and acceleration constraint equations applied to the multibody system can be written as:

$$
\begin{aligned}
& \boldsymbol{\Phi}(\mathbf{q}, t)=\mathbf{0} \\
& \dot{\boldsymbol{\Phi}}=\boldsymbol{\Phi}_{\mathbf{q}} \dot{\mathbf{q}}+\boldsymbol{\Phi}_{t}=\mathbf{0} \\
& \ddot{\boldsymbol{\Phi}}=\boldsymbol{\Phi}_{\mathbf{q}} \ddot{\mathbf{q}}+\dot{\boldsymbol{\Phi}}_{\mathbf{q}} \dot{\mathbf{q}}+\dot{\boldsymbol{\Phi}}_{t}=\mathbf{0}
\end{aligned}
$$

The $n$ Cartesian coordinates are linked by $m$ algebraic constraint equations. 
Only holonomic and scleronomic constraint equations (but possibly redundant, as long as they are compatible) are considered in this Thesis.

Equations (1.1)-(1.4) constitute a system of differential algebraic equations (DAEs) of index-3. If only Equations (1.1) and (1.4) are taken into account, the following index-1 DAEs are obtained:

$$
\underbrace{\left[\begin{array}{cc}
\mathbf{M} & \boldsymbol{\Phi}_{\mathbf{q}}^{\mathrm{T}} \\
\boldsymbol{\Phi}_{\mathbf{q}} & \mathbf{0}
\end{array}\right]}_{\mathbf{H}}\left\{\begin{array}{c}
\ddot{\mathbf{q}} \\
\boldsymbol{\lambda}
\end{array}\right\}=\left\{\begin{array}{c}
\mathbf{Q} \\
-\dot{\boldsymbol{\Phi}}_{\mathbf{q}} \dot{\mathbf{q}}
\end{array}\right\}
$$

It is common to find the hypothesis that the $n \times n$ matrix $\mathbf{M}$ is invertible and the $m \times n$ Jacobian matrix $\boldsymbol{\Phi}_{\mathbf{q}}$ has full-rank $m$. In this case, the solution of Equation (1.5) can be expressed compactly as follows:

$$
\begin{aligned}
& \boldsymbol{\lambda}=\left(\boldsymbol{\Phi}_{\mathbf{q}} \mathbf{M}^{-1} \boldsymbol{\Phi}_{\mathbf{q}}^{\mathrm{T}}\right)^{-1}\left(\boldsymbol{\Phi}_{\mathbf{q}} \mathbf{M}^{-1} \mathbf{Q}+\dot{\boldsymbol{\Phi}}_{\mathbf{q}} \dot{\mathbf{q}}\right) \\
& \ddot{\mathbf{q}}=\mathbf{M}^{-1}\left(\mathbf{Q}-\boldsymbol{\Phi}_{\mathbf{q}}^{\mathrm{T}} \boldsymbol{\lambda}\right)
\end{aligned}
$$

For the sake of generality, we consider the case in which the mass matrix is positive semi-definite, and also redundant constraint equations may exist. So Equations (1.6) and (1.7) are not applicable.

Equation (1.5) is well-known in the field of nonlinear optimization, where it is referred to as a Karush-Kuhn-Tucker (KKT) system [72, 79]. Matrix $\mathbf{H}$ on the left hand side of Equation (1.5) is called the optimization matrix [101, 108]. The system of Equation (1.5) involves a constraint stabilization problem, since only the acceleration level constraint equations are considered. The system positions and velocities provided by the integrator suffer from the "drift" problem. Two common solutions to solve this problem are the Baumgarte stabilization method $[11,21-23,39,81]$ and the mass-orthogonal projection method of system positions and velocities [1, 24, 26, 76, 89, 98].

A method that is essential for this Thesis will be presented next. It is based on 
the elimination of Lagrange multipliers.

\subsubsection{Null space method}

The null space method eliminates the Lagrange multipliers from the equations of motion. This is traditionally done by calculating a basis for the kernel or null space of the Jacobian matrix $\boldsymbol{\Phi}_{\mathbf{q}}[44,47,55,107]$. Let us recall Equation (1.5): we know that $m$ Cartesian coordinates are fixed by virtue of the acceleration constraint equations and $f$ (namely $n-m$ ) of them are actually free to satisfy dynamic equations. The Cartesian accelerations, therefore, can be partitioned as:

$$
\ddot{\mathbf{q}}=\left\{\begin{array}{c}
\ddot{\mathbf{q}}^{\mathrm{d}} \\
\ddot{\mathbf{q}}^{\mathrm{i}}
\end{array}\right\}
$$

where $\ddot{\mathbf{q}}^{\mathrm{d}} \in \mathbb{R}^{m}$ is kinematically determined by the acceleration constraint equations; $\ddot{\mathbf{q}}^{\mathrm{i}} \in \mathbb{R}^{f}$ is determined by the system dynamics. These are the dependent and independent subsets of vector $\ddot{\mathbf{q}}$. Similarly, the Jacobian matrix $\boldsymbol{\Phi}_{\mathbf{q}}$ can be partitioned into the dependent coordinate part $\Phi_{\mathbf{q}}^{\mathrm{d}} \in \mathbb{R}^{m \times m}$ and the independent coordinate part $\boldsymbol{\Phi}_{\mathbf{q}}^{\mathrm{i}} \in \mathbb{R}^{m \times f}$. This method is called the coordinate partitioning method $[102,112]$. To do this, the dependent coordinates should be chosen in such a way that matrix $\boldsymbol{\Phi}_{\mathbf{q}}^{\mathrm{d}}$ is invertible.

Thus, the acceleration level constraint equations in Equation (1.4) can be rewritten as:

$$
\left[\begin{array}{cc}
\boldsymbol{\Phi}_{\mathbf{q}}^{\mathrm{d}} & \boldsymbol{\Phi}_{\mathbf{q}}^{\mathrm{i}} \\
\mathbf{0}_{f \times n} & \mathbf{I}_{f}
\end{array}\right]\left\{\begin{array}{c}
\ddot{\mathbf{q}}^{\mathrm{d}} \\
\ddot{\mathbf{q}}^{\mathrm{i}}
\end{array}\right\}=\left\{\begin{array}{c}
-\dot{\boldsymbol{\Phi}} \dot{\mathbf{q}} \\
\ddot{\mathbf{q}}^{\mathrm{i}}
\end{array}\right\}
$$

The leading matrix in Equation (1.9) is invertible. If $[\mathbf{S} \mathbf{R}]$ is its inverse matrix, the following equations can be obtained [46]:

$$
\left[\begin{array}{cc}
\boldsymbol{\Phi}_{\mathbf{q}}^{\mathrm{d}} & \boldsymbol{\Phi}_{\mathbf{q}}^{\mathrm{i}} \\
\mathbf{0}_{f \times n} & \mathbf{I}_{f}
\end{array}\right]\left[\begin{array}{cc}
\mathbf{S}^{\mathrm{d}} & \mathbf{R}^{\mathrm{d}} \\
\mathbf{S}^{\mathrm{i}} & \mathbf{R}^{\mathrm{i}}
\end{array}\right]=\left[\begin{array}{cc}
\mathbf{I}_{m} & \mathbf{0} \\
\mathbf{0} & \mathbf{I}_{f}
\end{array}\right]
$$


where $\mathbf{R}^{\mathrm{d}}, \mathbf{S}^{\mathrm{d}}$ and $\mathbf{R}^{\mathrm{i}}, \mathbf{S}^{\mathrm{i}}$ correspond to the dependent and independent coordinate parts of $\mathbf{R}$ and $\mathbf{S}$. Matrices $\mathbf{R}$ and $\mathbf{S}$ take the following expressions:

$$
\begin{aligned}
& \mathbf{R}=\left[\begin{array}{c}
\mathbf{R}^{\mathrm{d}} \\
\mathbf{I}_{f}
\end{array}\right]=\left[\begin{array}{c}
-\left(\boldsymbol{\Phi}_{\mathbf{q}}^{\mathrm{d}}\right)^{-1} \boldsymbol{\Phi}_{\mathbf{q}}^{\mathrm{i}} \\
\mathbf{I}_{f}
\end{array}\right] \\
& \mathbf{S}=\left[\begin{array}{c}
\mathbf{S}^{\mathrm{d}} \\
\mathbf{0}_{f \times m}
\end{array}\right]=\left[\begin{array}{c}
\left(\boldsymbol{\Phi}_{\mathbf{q}}^{\mathrm{d}}\right)^{-1} \\
\mathbf{0}_{f \times m}
\end{array}\right]
\end{aligned}
$$

We can see that the columns of matrix $\mathbf{R}$ are a basis of $\operatorname{ker}\left(\boldsymbol{\Phi}_{\mathbf{q}}\right)$ and matrix $\mathbf{S}$ is a right inverse of $\boldsymbol{\Phi}_{\mathbf{q}}$. It is then verified that:

$$
\begin{aligned}
& \boldsymbol{\Phi}_{\mathbf{q}} \mathbf{R}=\mathbf{0}_{m \times f} \\
& \boldsymbol{\Phi}_{\mathbf{q}} \mathbf{S}=\mathbf{I}_{m}
\end{aligned}
$$

By premultiplying Equation (1.1) with matrix $\mathbf{R}^{\mathrm{T}}$ and taking into account Equation (1.13), the Lagrange multipliers are eliminated:

$$
\mathbf{R}^{\mathrm{T}} \mathbf{M} \ddot{\mathbf{q}}=\mathbf{R}^{\mathrm{T}} \mathbf{Q}
$$

Hence, the equations of motion through the null space method are:

$$
\left[\begin{array}{c}
\mathbf{R}^{\mathrm{T}} \mathbf{M} \\
\boldsymbol{\Phi}_{\mathbf{q}}
\end{array}\right] \ddot{\mathbf{q}}=\left\{\begin{array}{c}
\mathbf{R}^{\mathrm{T}} \mathbf{Q} \\
-\dot{\boldsymbol{\Phi}} \dot{\mathbf{q}}
\end{array}\right\}
$$

The Lagrange multipliers have been eliminated from the equations of motion, and thus Equation (1.16) is smaller. However, these equations are not very different from the index-1 Lagrange's equations. They suffer from the same instability problem in the numerical integration process, since they do not consider the position and velocity constraints either [44, 46, 108]. 


\subsection{State of the art of topological formulations}

The aforementioned classical formulations are two examples of global formulations that use absolute or reference-point coordinates and consider both open- and closed-loop systems in exactly the same way. Laulusa and Bauchau [75] presented a comprehensive review of the theoretical foundations for the enforcement of constraints in multibody systems. They assessed their accuracy and efficiency, underlined the relationship among the formulations and recommended those that seem to perform best. In this Thesis, the focus is placed on topological formulations, which are considered to be more efficient for medium-large multibody systems, although there exist examples of numerically efficient global formulations.

In recent decades, many efficient topological formulations have been proposed for both open- and closed-loop multibody systems. Haug, Negrut, Serban et al. [54, $80,99,101]$ introduced a topological formulation in terms of relative coordinates for tree structure systems. They also presented the equations of motion of closed chain systems by imposing a set of acceleration level loop-closure constraint equations. Saha [93] presented the equations of motion of serial systems with the aid of the decoupled natural orthogonal complement matrices associated with the velocity constraints. Later, Saha, Khan et al. [63, 64, 90, 94] proposed a recursive formulation for parallel systems based on relative coordinates for the inverse and forward dynamic control and simulation. These topological formulations have been proven efficient in small-medium multibody systems [54, 93, 94, 101].

Bae et al. [13-15, 18, 19, 91] introduced a generalized semi-recursive formulation for closed-loop systems through the combination of dynamic equations, kinematic constraint equations for positions, velocities and accelerations and an implicit integrator based on the backward differentiation formulae. The tangent matrix was computed exactly using forward and backward recursive formulas. Kim et al. $[65-68,70]$ presented a subsystem synthesis method for the vehicle dynamics. In this method, each subsystem was independently analyzed, and then synthesized 
to the chassis with its effective inertia matrix and force vector for the global vehicle system analysis. In this way, the equations of motion of the chassis can be formed and independently solved. Thereafter, the equations of motion of each subsystem can be solved based on the known accelerations of the chassis. García de Jalón et al. [43, 46, 57, 92] proposed a double-step semi-recursive formulation in terms of independent relative coordinates. The first velocity transformation was used to express Cartesian coordinates in terms of dependent relative coordinates. By introducing the second velocity transformation, a set of independent relative coordinates was extracted from the dependent ones, which then allowed one to integrate the equations of motion using Maggi's equations in a fairly efficient way.

\subsection{Double-step semi-recursive formulation}

An alternative semi-recursive formulation was proposed by García de Jalón et al. $[43,46,92]$. We refer to this method as the double-step semi-recursive formulation (DS-SRF) because it is based on two velocity transformations that we refer to as steps. The first one allows one to write the dynamic equations of open-loop systems, and the second one imposes loop-closure constraints.

\subsubsection{Open-loop equations}

The system is first considered as an open-loop multibody system, which sometimes requires the temporary cutting of certain joints. The connection between rigid bodies through revolute $(\mathrm{R})$ and prismatic $(\mathrm{P})$ joints can be modeled as shown in Figure 1.1. Other kinds of kinematic joints with more than one degrees of freedom (DOF) can be broken down into a series of massless auxiliary bodies connected by basic 1-DOF joints. The inboard (or input) joint in each body is defined as the joint that is closest to the base body in the spanning tree. Each body has one inboard joint and possibly one or more outboard (or output) joints. 

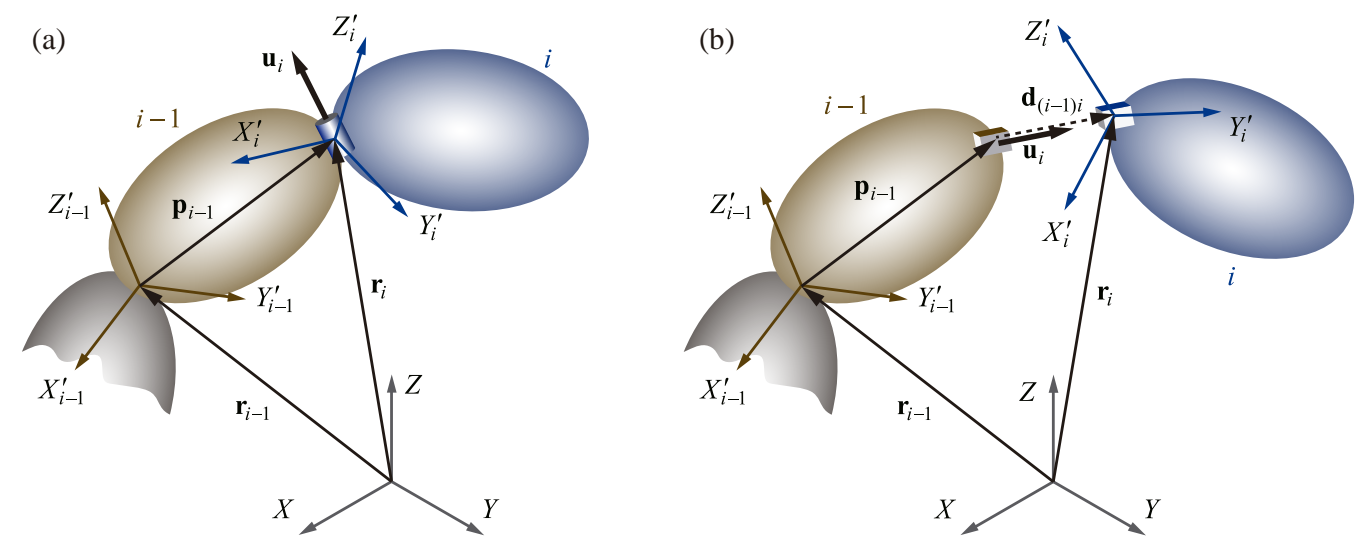

Figure 1.1: (a) Revolute and (b) Prismatic joints (courtesy of A. Callejo)

The following Cartesian variables are used to define the global (or absolute) velocity and acceleration of each body:

$$
\mathbf{Z}_{i} \equiv\left\{\begin{array}{c}
\dot{\mathbf{s}}_{i} \\
\boldsymbol{\omega}_{i}
\end{array}\right\}, \quad \dot{\mathbf{Z}}_{i} \equiv\left\{\begin{array}{c}
\ddot{\mathbf{s}}_{i} \\
\dot{\boldsymbol{\omega}}_{i}
\end{array}\right\}
$$

where $\dot{\mathbf{s}}_{i}$ and $\ddot{\mathbf{s}}_{i}$ are the velocity and acceleration of the point attached to body $i$ that instantaneously coincides with the origin of the global reference frame. The sharing of the reference point simplifies some of the following equations. The global velocities and accelerations can be recursively expressed in terms of those of the inboard body:

$$
\begin{aligned}
\mathbf{Z}_{i} & =\mathbf{Z}_{i-1}+\mathbf{b}_{i} \dot{z}_{i} \\
\dot{\mathbf{Z}}_{i} & =\dot{\mathbf{Z}}_{i-1}+\mathbf{b}_{i} \ddot{z}_{i}+\mathbf{d}_{i}
\end{aligned}
$$

where scalar $\dot{z}_{i}$ is the time rate of change of the joint coordinate and vectors $\mathbf{b}_{i}$ and $\mathbf{d}_{i}$ depend on the type of joint.

Then, a velocity transformation matrix, $\mathbf{R} \in \mathbb{R}^{6 n \times n}$, can be introduced [61]. This matrix maps the absolute velocities into a set of relative velocities. In other words, the absolute velocities are expressed as a linear combination of the columns 
of matrix R; through differentiation, an expression can also be obtained for the accelerations:

$$
\begin{aligned}
& \mathbf{Z}=\mathbf{R}_{1} \dot{z}_{1}+\mathbf{R}_{2} \dot{z}_{2}+\cdots+\mathbf{R}_{n} \dot{z}_{n}=\mathbf{R} \dot{\mathbf{z}}=\mathbf{T} \mathbf{R}_{\mathrm{d}} \dot{\mathbf{z}} \\
& \dot{\mathbf{Z}}=\mathbf{T R}_{\mathrm{d}} \ddot{\mathbf{z}}+\mathbf{T} \dot{\mathbf{R}}_{\mathrm{d}} \dot{\mathbf{z}}
\end{aligned}
$$

where array $\mathbf{Z} \in \mathbb{R}^{6 n}$ containts the dependent global velocities; vector $\dot{\mathbf{z}} \in \mathbb{R}^{n}$ is the vector of dependent relative velocities, whose elements are the coefficients of the linear combination; matrix $\mathbf{T} \in \mathbb{R}^{6 n \times 6 n}$ is the so-called path matrix, representing the system topology of the open-loop multibody system; and matrix $\mathbf{R}_{\mathrm{d}} \in \mathbb{R}^{6 n \times n}$ is a block-diagonal matrix. The $j$ th column of matrix $\mathbf{R}$ can be computed recursively: its elements correspond to Cartesian velocities of the bodies that are upwards in the topology tree, originated by a unit relative velocity in joint $j$ and null relative velocities in the remaining ones [43]. Note that matrix $\mathbf{R}$ need not be calculated explicitly in the computer code.

The dynamic equations of the open-loop system can be expressed in terms of dependent relative coordinates by using the principle of virtual power and substituting Equation (1.20) and its time derivative [61, 108, 115]:

$$
\mathbf{R}_{\mathrm{d}}^{\mathrm{T}} \mathbf{T}^{\mathrm{T}} \overline{\mathbf{M}} \mathbf{T} \mathbf{R}_{\mathrm{d}} \ddot{\mathbf{z}}=\mathbf{R}_{\mathrm{d}}^{\mathrm{T}} \mathbf{T}^{\mathrm{T}}(\overline{\mathbf{Q}}-\overline{\mathbf{P}})
$$

where $\ddot{\mathbf{z}} \in \mathbb{R}^{n}$ represents the array of dependent relative accelerations of the system and terms $\overline{\mathbf{M}} \in \mathbb{R}^{6 n \times 6 n}, \overline{\mathbf{Q}} \in \mathbb{R}^{6 n}$ and $\overline{\mathbf{P}} \in \mathbb{R}^{6 n}$ are, respectively, the global inertia matrix, applied forces and velocity-dependent inertia forces. The upper bar simply indicates reference to the global reference frame described after Equation (1.17). 
Equation (1.22) can be rewritten in compact notation as:

$$
\begin{aligned}
\mathbf{R}_{\mathrm{d}}^{\mathrm{T}} \mathbf{M}^{\Sigma} \mathbf{R}_{\mathrm{d}} \ddot{\mathbf{z}} & =\mathbf{R}_{\mathrm{d}}^{\mathrm{T}}\left(\mathbf{Q}^{\Sigma}-\mathbf{P}^{\Sigma}\right) \\
\mathbf{M}^{\Sigma} & \equiv \mathbf{T}^{\mathrm{T}} \overline{\mathbf{M}} \mathbf{T} \\
\mathbf{Q}^{\Sigma} & \equiv \mathbf{T}^{\mathrm{T}} \overline{\mathbf{Q}} \\
\mathbf{P}^{\Sigma} & \equiv \mathbf{T}^{\mathrm{T}} \overline{\mathbf{P}}
\end{aligned}
$$

where terms $\mathbf{M}^{\Sigma} \in \mathbb{R}^{6 n \times 6 n}, \mathbf{Q}^{\Sigma} \in \mathbb{R}^{6 n}$ and $\mathbf{P}^{\Sigma} \in \mathbb{R}^{6 n}$ are the accumulated variables corresponding to $\overline{\mathbf{M}}, \overline{\mathbf{Q}}$ and $\overline{\mathbf{P}}[43,46]$.

\subsubsection{Closed-loop equations}

A set of $m$ loop-closure constraint equations $(\boldsymbol{\Phi}(\mathbf{z})=\mathbf{0})$ is now considered in order to close the cut-joints and address the dynamics of the closed-loop system. To simplify the analysis, constraint equations are assumed to be holonomic and scleronomic (but possibly redundant, as long as they are compatible).

Figure 1.2 depicts the opening of closed chains by removing a rod and by cutting spherical and revolute joints. The loop-closure constraint equations are first established through Cartesian coordinates of points (r) and Cartesian components of unit vectors ( $\mathbf{u}$ ) that belong to the bodies at hand. The Jacobian matrix of the loop-closure constraints with respect to the relative coordinates, $\boldsymbol{\Phi}_{\mathbf{z}}$, can be computed by using the chain rule. In the case of a spherical joint between point $j$ of body $j$ and point $k$ of body $k$, the loop-closure constraint equations can be differentiated as:

$$
\boldsymbol{\Phi}_{\mathbf{z}}=\boldsymbol{\Phi}_{\mathbf{r}_{j}} \frac{\partial \mathbf{r}_{j}}{\partial \mathbf{z}}+\boldsymbol{\Phi}_{\mathbf{r}_{k}} \frac{\partial \mathbf{r}_{k}}{\partial \mathbf{z}}=\boldsymbol{\Phi}_{\mathbf{r}_{j}} \frac{\partial \dot{\mathbf{r}}_{j}}{\partial \dot{\mathbf{z}}}+\boldsymbol{\Phi}_{\mathbf{r}_{k}} \frac{\partial \dot{\mathbf{r}}_{k}}{\partial \dot{\mathbf{z}}}
$$

where $\boldsymbol{\Phi}_{\mathbf{r}}$ represents the partial derivative of the constraints with respect to the Cartesian coordinates, which can be easily computed; $\partial \mathbf{r} / \partial \mathbf{z}$ represents the partial derivative of Cartesian coordinates with respect to the dependent relative coordinates, which can be obtained through the open-loop recursive kinematics $[43,46]$. 


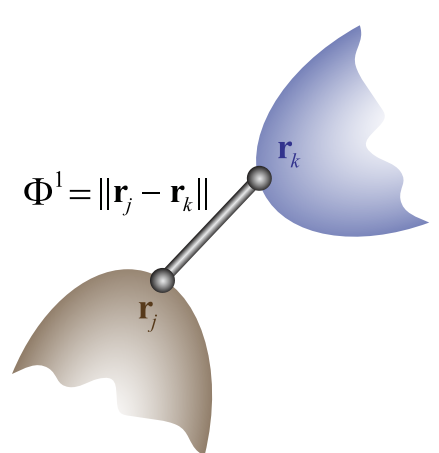

(a) Rod

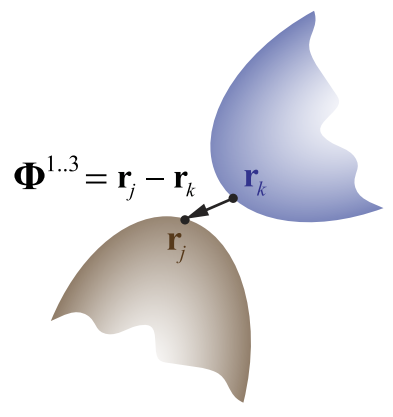

(b) Spherical joint

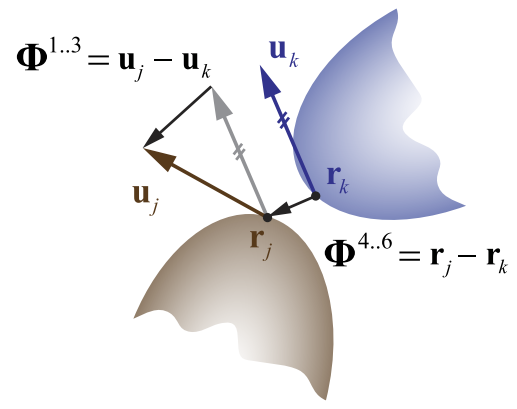

(c) Revolute joint

Figure 1.2: Rod, spherical joint and revolute joint constraint equations; note that only 5 out of 6 equations are independent in the revolute joint case (courtesy of A. Callejo)

The closed-loop dynamic equations can be formulated by imposing the loopclosure constraint equations into the open-loop dynamic equations using Lagrange multipliers:

$$
\mathbf{R}_{\mathrm{d}}^{\mathrm{T}} \mathbf{M}^{\Sigma} \mathbf{R}_{\mathrm{d}} \ddot{\mathbf{z}}+\boldsymbol{\Phi}_{\mathbf{z}}^{\mathrm{T}} \boldsymbol{\lambda}=\mathbf{R}_{\mathrm{d}}^{\mathrm{T}}\left(\mathbf{Q}^{\Sigma}-\mathbf{P}^{\Sigma}\right)
$$

An alternative way to do so is to introduce a second velocity transformation, $\mathbf{R}_{\mathrm{z}} \in \mathbb{R}^{n \times f}$, in order to transform the dependent relative coordinates into independent ones $[43,46]$. This way, the Lagrange multipliers associated with the loop-closure constraint equations are eliminated from the equations of motion. The second velocity transformation can be formulated as follows [46, 71, 75, 86, 111]:

$$
\begin{aligned}
& \dot{\mathbf{z}}=\left[\begin{array}{c}
-\left(\boldsymbol{\Phi}_{\mathbf{z}}^{\mathrm{d}}\right)^{-1} \boldsymbol{\Phi}_{\mathbf{z}}^{\mathrm{i}} \\
\mathbf{I}
\end{array}\right] \dot{\mathbf{z}}^{\mathrm{i}} \equiv \mathbf{R}_{\mathbf{z}} \dot{\mathbf{z}}^{\mathrm{i}} \\
& \ddot{\mathbf{z}}=\mathbf{R}_{\mathbf{z}} \ddot{\mathbf{z}}^{\mathrm{i}}+\dot{\mathbf{R}}_{\mathrm{z}} \dot{\mathbf{z}}^{\mathrm{i}}
\end{aligned}
$$

where the second expression has been obtained through differentiation; matrices $\boldsymbol{\Phi}_{\mathbf{z}}^{\mathrm{d}} \in \mathbb{R}^{m \times m}$ and $\boldsymbol{\Phi}_{\mathbf{z}}^{\mathrm{i}} \in \mathbb{R}^{m \times f}$ contain the dependent and independent columns of the constraint Jacobian; $\dot{\mathbf{z}}^{\mathrm{i}}, \ddot{\mathbf{z}}^{\mathrm{i}} \in \mathbb{R}^{f}$ are the independent relative velocities and accelerations; and $f$ is the number of independent coordinates or DOFs. The 
selection of independent relative coordinates is assumed to be valid throughout the entire simulation, which is typically the case in vehicle dynamics [100]. We can see that $\mathbf{R}_{\mathrm{Z}}$ is, in fact, a basis of the Jacobian matrix null space.

Introducing the second velocity transformation into Equation (1.28), the final form of the equations of motion in terms of independent relative coordinates is obtained:

$$
\begin{array}{r}
\mathbf{R}_{\mathrm{z}}^{\mathrm{T}} \mathbf{R}_{\mathrm{d}}^{\mathrm{T}} \mathbf{M}^{\Sigma} \mathbf{R}_{\mathrm{d}} \mathbf{R}_{\mathrm{z}} \ddot{\mathbf{z}}^{\mathrm{i}}=\mathbf{R}_{\mathrm{z}}^{\mathrm{T}} \mathbf{R}_{\mathrm{d}}^{\mathrm{T}}\left(\mathbf{Q}^{\Sigma}-\mathbf{T}^{\mathrm{T}} \overline{\mathbf{M}} \mathbf{D}\right) \\
\mathbf{D} \equiv \mathbf{T} \mathbf{R}_{\mathrm{d}}\left\{\begin{array}{c}
-\left(\boldsymbol{\Phi}_{\mathbf{z}}^{\mathrm{d}}\right)^{-1}\left(\dot{\boldsymbol{\Phi}}_{\mathbf{z}} \dot{\mathbf{z}}\right) \\
\mathbf{0}
\end{array}\right\}+\mathbf{T} \dot{\mathbf{R}}_{\mathrm{d}} \dot{\mathbf{z}}
\end{array}
$$

where $\mathbf{D} \in \mathbb{R}^{6 n}$ contains the absolute accelerations corresponding to null independent relative accelerations (see Equation (1.21)). Further details and proof of these equations can be found in $[43,46,92]$. The key aspect is that Equation (1.31) now constitutes a system of ordinary differential equations with only one equation per DOF. This approach corresponds to a state-space formulation of the equations of motion via the embedding or matrix-R method [43, 46, 92].

Although the basis of the Jacobian matrix null space needs to be recomputed every time-step, the resulting linear system is considerably smaller $(f)$ than the one that would be obtained through traditional index-1 approaches with Lagrange multipliers $(n+m)$. The main advantages of this formulation are the efficient implementation of recursive kinematics, the robust enforcement of constraint equations, the simplicity of the formulation and the ordinary differential nature of the resulting equations, which enables the use of standard integrators.

\subsection{Challenges and motivation}

The improvement of computational efficiency is a very challenging topic in the field of multibody system dynamics. Moreover, it is difficult to compare the efficiency 
of different topological formulations, which appears to be hindered by the difficulty to compare different numerical examples, computer program implementations and numerical formulations. Yet, robust and efficient topological formulations for the simulation of multibody systems are essential for many applications such as real-time dynamics, contact problems, parameter identification and dynamic response optimization $[6,7,20,28-30,38,73,74]$.

In order to partially address the challenges, this Thesis presents a comprehensively comparative study of semi-recursive and subsystem synthesis algorithms for the efficient simulation of multibody systems, and introduces several efficient techniques to improve the efficiency of the double-step semi-recursive formulation. On the other hand, systems with multiple rods or systems depending more heavily on rod inertial forces can greatly benefit from special modeling techniques.

In short, even though the theory of rigid-body systems has been well-established for centuries, the efficient computational implementation of complex multibody systems is still a challenging topic $[95,96]$. Recent publications on this matter outline the necessity of going further into this topic [37, 41, 42, 49, 53, 56]. These publications have served as basic references during the development of this Thesis. In the Thesis, an attempt is made to evaluate and improve the computational efficiency of several state-of-the-art topological formulations for medium-large vehicle models.

\subsection{Objectives and structure}

The structure of this Thesis, which is determined by the papers prepared during the Thesis development, is presented next, in accordance with the Thesis objectives:

- Implement the double-step semi-recursive formulation for the real-time simulation of vehicle dynamics. Implement a 16-DOF sedan vehicle model, a 28-DOF rover model and a 40-DOF semi-trailer truck model. These topics 
are covered in Chapters 2, 3 and 4.

- Compare three state-of-the-art topological formulations, namely generalized semi-recursive, subsystem synthesis and double-step semi-recursive formulations. Analyze their solution accuracy and computational efficiency in a systematic way based on the aforementioned vehicle models. Chapter 2 is devoted to these objectives, corresponding to a submitted paper [31].

- Apply an approximation method for the rod-related inertial forces to further improve the computational efficiency. Implement an additional partitioning of the rod inertia to improve the solution accuracy without efficiency loss. These tasks are undertaken in Chapter 3, corresponding to a published paper [85].

- Introduce an iterative refinement technique onto the explicit 4th-order RungeKutta integrator to improve the computational efficiency. Reuse the generalized mass matrix factorization between neighboring Runge-Kutta steps for efficient iterative refinement. Assess the accuracy and efficiency using the aforementioned vehicle models. This part is carried out in Chapter 4, corresponding to a prepared paper.

- Lastly, conclude the contributions to the double-step semi-recursive formulation. Set out the possible objectives for further development (Chapter $5)$. 


\section{Chapter 2}

\section{Comparison of semi-recursive and subsystem synthesis algorithms}

A great variety of formulations exist for the numerical simulation of rigid-body systems, particularly of medium-large systems such as vehicles. Topological formulations, which are considered to be the most efficient ones, are often cumbersome and not necessarily easy to implement. As a consequence, there is a lack of comparative evidence to support the performance of these formulations. In this chapter, we present and compare three state-of-the-art topological formulations for multibody dynamics: generalized semi-recursive, double-step semi-recursive (Chapter 1.3) and subsystem synthesis methods. We analyze the background, underlying principles, numerical efficiency and accuracy of these formulations in a systematic way. A 28-DOF, open-loop rover model and a 16-DOF, closed-loop sedan car model are selected as study cases. Insight on the key aspects toward performance is provided.

\subsection{Introduction}

The evaluation of computational efficiency in the area of multibody dynamics appears to have been hindered by the difficulty to compare different numerical 
examples, computer program implementations and numerical formulations. Often, authors analyze only the examples that highlight the efficiency of their particular formulation. Reproducing and validating results reported by other researchers requires enormous amounts of coding and expertise.

In recent years, there has been a growing interest in the concept of benchmark problems within the dynamic simulation of multibody systems [50, 84, 97]. Similar to other areas of computational mechanics, such as the finite element method, researchers have increasingly been developing numerical examples suitable for comparison and validation. Such examples greatly facilitate the comparison of otherwise complex algorithms and mechanical systems. One of the key characteristics of benchmark problems is the ability to analyze the computational efficiency of different numerical approaches applied to the same numerical example.

Following these principles, we present a thorough comparison of three of the most efficient multibody formulations. The mechanical systems hereby studied are medium-large open- and closed-loop vehicles. We believe these systems are representative of multibody systems often found in industry. Specifically, we selected a 28-DOF, open-loop rover model and a 16-DOF, closed-loop sedan vehicle model as test cases.

There are abundant methods for the expression and solution of the dynamic equations that govern rigid-body systems. The most commonly used ones stem from Lagrange's equations of the first kind:

$$
\mathbf{M} \ddot{\mathbf{q}}-\boldsymbol{\Phi}_{\mathbf{q}}^{\mathrm{T}} \lambda=\mathbf{Q}
$$

where $\mathbf{q} \in \mathbb{R}^{n}$ is the array of generalized coordinates that represent the system position; $\mathbf{M}(\mathbf{q}) \in \mathbb{R}^{n \times n}$ is the mass matrix; $\mathbf{Q}(\mathbf{q}, \dot{\mathbf{q}}) \in \mathbb{R}^{n}$ is the array of generalized applied forces and velocity-dependent inertia forces; $\boldsymbol{\Phi}(\mathbf{q}, t)=\mathbf{0} \in \mathbb{R}^{m}$ is the array of holonomic constraint equations; $\boldsymbol{\Phi}_{\mathbf{q}} \in \mathbb{R}^{m \times n}$ is its Jacobian matrix; $\boldsymbol{\lambda} \in \mathbb{R}^{m}$ is the array of Lagrange multipliers associated with the constraints. 
Multibody formulations differ from one another in the specific form of the equations of motion, the type of coordinates, the parameterization of rotations, the constraint stabilization technique, the time integration algorithm and the computer implementation, among others. They are generally classified as global or topological. Global formulations are usually based on absolute coordinates and the direct, systematic solution of the equations of motion. Topological formulations, on the other hand, are usually based on relative coordinates and take advantage of the system structure and connectivity to formulate the equations of motion. Even though there exist examples of numerically efficient global formulations, the topological ones are generally considered to be more efficient, especially for large multibody systems. The three formulations analyzed in this Chapter are considered to be topological formulations.

Most recursive topological methods originated within the field of robotics, seeking the solution of both inverse dynamics and forward dynamics problems for open-loop systems. Some of them require the solution of sets of linear equations, such as the composite-body method from Walker and Orin [110] and the so-called semi-recursive methods introduced by Jerkovsky $[12,61]$. Some, on the other hand, do not require the solution of such linear systems, for instance the very well known articulated-body method from Featherstone [35]. These methods are often referred to as order- $N$ (where $N$ is the number of bodies) or fully-recursive methods, and are considered to be the most efficient ones for open-loop systems where $N>11$ [35] or $N>7$ [105].

The extension of recursive methods to closed-loop systems can be done through the concept of cut-joints. Namely, certain joints and their corresponding constraint equations are eliminated to consider the system as an open-loop system and are then introduced into the formulation using recursive or numeric constraint enforcement techniques. Even though some fully-recursive approaches for closed-loop systems have been presented [18], they are considerably more complicated and present significant numerical and implementation issues. Alternatively, the cutjoint constraints can be numerically enforced through penalty terms, stabilization 
methods, projection methods, etc. (see [44]).

In this Chapter we analyze two semi-recursive formulations and a subsystem synthesis method. First, we present the theoretical background of the methods using a unified notation, which we believe is important for a meaningful comparison. Second, we carry out detailed numerical simulations to assess their computational efficiency. In order to conduct a fair comparison, all formulations are implemented in the same computer language, tested using the same numerical examples and run in the same machine.

Note that numerical efficiency is hereby considered as the total amount of time that simulations require, rather than the theoretical count of arithmetic operations. Elapsed times in modern computers heavily depend on CPU architectures and coding practices, and thus they are not exclusively related to the number of arithmetic operations. This is especially true in algorithms where $N$ is not large, such as the ones at hand.

Throughout the Chapter, different kinematic expressions that depend on the joint type will arise; for the sake of brevity, we will only present the revolute joint expressions. Expressions for alternative 1-DOF joint types are similar and can easily be derived.

\subsection{Generalized semi-recursive formulation}

Semi-recursive formulations were introduced by Jerkovsky [61] and later extended by other authors $[69,80]$. They are based on the concept of velocity transformation. In the late 90s, Bae et al. developed a semi-recursive method [13-15, 18, 19, 91] that was related to Yen's integrator [116] and was specifically designed to deal with stiff problems. We refer to this method as the generalized semi-recursive formulation (G-SRF). 


\subsubsection{Dynamic equations}

Let us consider the multibody system as a tree structure with an associated spanning tree. The G-SRF employs local reference frames located on the inboard joints, as shown in Figure 2.1. The inboard joint in each body is defined as the joint that is closest to the base body in the spanning tree. Each body has one inboard joint and possibly one or more outboard joints. The motion, constraint, and integrator equations are solved for every integration step via a self-adjusting modified Newton Raphson process until convergence. The key aspect of the G-SRF is the efficient evaluation of Jacobian terms using recursive formulas for every Jacobian matrix term.

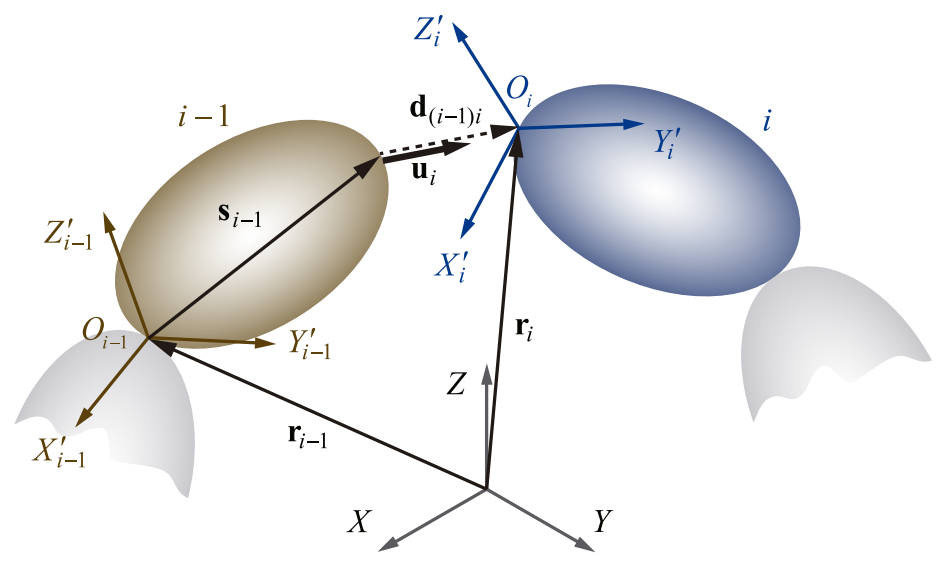

Figure 2.1: Kinematic relationship between two connected bodies

Let us define $(n+1)$ reference frames in a system with $n$ bodies: one global frame, and one local frame per body located on the inboard joint, as shown in Figure 2.1. Let us define the local velocities of the body:

$$
\mathbf{Y}_{i}=\left\{\begin{array}{c}
\dot{\mathbf{r}}_{i}^{\prime} \\
\boldsymbol{\omega}_{i}^{\prime}
\end{array}\right\}=\left\{\begin{array}{c}
\mathbf{A}_{i}^{\mathrm{T}} \dot{\mathbf{r}}_{i} \\
\mathbf{A}_{i}^{\mathrm{T}} \boldsymbol{\omega}_{i}
\end{array}\right\}
$$

where $\dot{\mathbf{r}}_{i}^{\prime}$ and $\boldsymbol{\omega}_{i}^{\prime}$ represent the Cartesian components of the velocity of the center of gravity and the angular velocity of body $i$ in the local reference frame; $\dot{\mathbf{r}}_{i}$ and 
$\boldsymbol{\omega}_{i}$ represent the corresponding Cartesian coordinates with respect to the global reference frame; and matrix $\mathbf{A}_{i}$ is the rotation matrix between both frames. The use of local coordinates leads to constant mass matrices, but brings about the need to differentiate the (numerous) rotation matrices.

Both linear and angular velocities can be expressed in the local reference frame in terms of the generalized velocities of the inboard body and a term that depends on the corresponding relative coordinate:

$$
\mathbf{Y}_{i}=\overbrace{\left[\begin{array}{cc}
\mathbf{A}_{(i-1) i} & -\mathbf{A}_{(i-1) i} \tilde{\mathbf{s}}_{i-1}^{\prime} \\
\mathbf{0} & \mathbf{A}_{(i-1) i}
\end{array}\right]}^{\mathbf{B}_{i}^{\mathrm{R}}} \mathbf{Y}_{i-1}+\overbrace{\left\{\begin{array}{c}
\mathbf{0} \\
\mathbf{A}_{(i-1) i} \mathbf{u}_{i}^{\prime}
\end{array}\right\}}^{\mathbf{b}_{i}^{\mathrm{R}}} \dot{z}_{i}
$$

where $z_{i}$ represents the relative coordinate between bodies $i$ and $(i-1) ; \mathbf{A}_{(i-1) i}$ is the rotation matrix between the local reference frames of bodies $i$ and $(i-1)$; vector $\mathbf{s}_{i-1}^{\prime}$ is the vector from the inboard joint to the outboard joint of body $(i-1)$; vector $\mathbf{u}_{i}^{\prime}$ is the unit vector along the axis of joint $i$; and the upper tilde represents the skew symmetric matrix associated with the cross product operator. Different joints will lead to different expressions for the above equations; for the sake of brevity, we will focus on the revolute case.

By differentiating Equation (2.3) with respect to time, we obtain an equivalent expression for the accelerations:

$$
\begin{gathered}
\dot{\mathbf{Y}}_{i}=\overbrace{\left[\begin{array}{cc}
\mathbf{A}_{(i-1) i} & \left.-\mathbf{A}_{(i-1) i} \tilde{\mathbf{s}}_{i-1}^{\prime}\right] \\
\mathbf{0} & \mathbf{A}_{(i-1) i}
\end{array}\right]}^{\mathbf{B}_{i}^{\mathrm{R}}} \dot{\mathbf{Y}}_{i-1}+\overbrace{\left\{\begin{array}{c}
\mathbf{0} \\
\mathbf{A}_{(i-1) i} \mathbf{u}_{i}^{\prime}
\end{array}\right\}}^{\mathbf{b}_{i}^{\mathrm{R}}} \ddot{z}_{i} \\
+\overbrace{\left\{\begin{array}{c}
\mathbf{A}_{(i-1) i} \tilde{\boldsymbol{\omega}}_{i-1} \tilde{\boldsymbol{\omega}}_{i-1} \mathbf{s}_{i-1}^{\prime} \\
-\dot{z}_{i} \mathbf{A}_{(i-1) i} \tilde{\mathbf{u}}^{\prime} \boldsymbol{\omega}_{i-1}^{\prime}
\end{array}\right\}}^{\mathbf{s}_{i-1}}
\end{gathered}
$$

By introducing a velocity transformation $\mathbf{B} \in \mathbb{R}^{6 n \times n}$, the velocities and acceler- 
ations of the whole system $\left(\mathbf{Y} \in \mathbb{R}^{6 n}\right.$ and $\left.\dot{\mathbf{Y}} \in \mathbb{R}^{6 n}\right)$ can be expressed in terms of the relative velocities and accelerations:

$$
\begin{aligned}
\mathbf{Y}(\mathbf{z}, \dot{\mathbf{z}}) & =\mathbf{B}(\mathbf{z}) \dot{\mathbf{z}} \\
\dot{\mathbf{Y}}(\mathbf{z}, \dot{\mathbf{z}}) & =\mathbf{B}(\mathbf{z}) \ddot{\mathbf{z}}+\dot{\mathbf{B}}(\mathbf{z}) \dot{\mathbf{z}}
\end{aligned}
$$

where $\mathbf{z}, \dot{\mathbf{z}}, \ddot{\mathbf{z}} \in \mathbb{R}^{n}$ contain the relative coordinates, velocities and accelerations of the entire system.

One can now apply the principle of virtual power and Lagrange multiplier methods to obtain the closed-loop dynamic equations in absolute coordinates:

$$
\mathbf{B}^{\mathrm{T}}(\mathbf{M} \dot{\mathbf{Y}}-\mathbf{Q})+\boldsymbol{\Phi}_{\mathbf{z}}^{\mathrm{T}} \boldsymbol{\lambda}=\mathbf{0}
$$

where $\mathbf{M} \in \mathbb{R}^{n \times n}$ and $\mathbf{Q} \in \mathbb{R}^{n}$ are the mass matrix and applied forces (including velocity-dependent inertia forces), and matrix $\boldsymbol{\Phi}_{\mathbf{z}} \in \mathbb{R}^{m \times n}$ is the Jacobian matrix of the loop-closure constraints with respect to the relative coordinates, which can be derived from $\boldsymbol{\Phi}_{\mathbf{Y}}$ by using the chain rule.

By introducing Equations (2.5) and (2.6) into Equation (2.7) and defining $\mathbf{p}^{\mathrm{T}} \equiv\left\{\mathbf{z}^{\mathrm{T}}, \dot{\mathbf{z}}^{\mathrm{T}}, \ddot{\mathbf{z}}^{\mathrm{T}}, \boldsymbol{\lambda}^{\mathrm{T}}\right\}$, the dynamic equations for the closed-loop system can be expressed in relative coordinates as:

$$
\mathbf{F}(\mathbf{p}) \equiv \mathbf{B}^{\mathrm{T}}(\mathbf{M B} \ddot{\mathbf{z}}+\mathbf{M} \dot{\mathbf{B}} \dot{\mathbf{z}}-\mathbf{Q})+\boldsymbol{\Phi}_{\mathbf{z}}^{\mathrm{T}} \boldsymbol{\lambda}=\mathbf{0}
$$

where $\mathbf{F}(\mathbf{p}) \in \mathbb{R}^{n}$ has been defined for convenience.

The closed-loop dynamic equations can be expressed by grouping Equation (2.8), the loop-closure constraint equations, and a set of backward differ- 
entiation formulas [13-15, 19]:

$$
\mathbf{H}(\mathbf{p}) \equiv\left\{\begin{array}{c}
\mathbf{F}(\mathbf{p}) \\
\boldsymbol{\Phi}(\mathbf{z}) \\
\dot{\boldsymbol{\Phi}}(\mathbf{z}, \dot{\mathbf{z}}) \\
\ddot{\boldsymbol{\Phi}}(\mathbf{z}, \dot{\mathbf{z}}, \ddot{\mathbf{z}}) \\
\mathbf{U}_{0}^{\mathrm{T}}\left(\dot{\mathbf{z}} h / b_{0}-\mathbf{z}-\boldsymbol{\eta}_{1}\right) \\
\mathbf{U}_{0}^{\mathrm{T}}\left(\ddot{\mathbf{z}} h / b_{0}-\dot{\mathbf{z}}-\boldsymbol{\eta}_{2}\right)
\end{array}\right\}=\mathbf{0}
$$

where $\boldsymbol{\Phi}, \dot{\boldsymbol{\Phi}}, \ddot{\boldsymbol{\Phi}} \in \mathbb{R}^{m}$ represent the loop-closure constraint equations at the position, velocity and acceleration levels; $\mathbf{U}_{0}^{\mathrm{T}}\left(\dot{\mathbf{z}} h / b_{0}-\mathbf{z}-\boldsymbol{\eta}_{1}\right) \in \mathbb{R}^{n}$ and $\mathbf{U}_{0}^{\mathrm{T}}\left(\ddot{\mathbf{z}} h / b_{0}-\dot{\mathbf{z}}-\boldsymbol{\eta}_{2}\right) \in$ $\mathbb{R}^{n}$ are the backward differentiation formulas that are used as an implicit integrator [15]; and $\boldsymbol{\eta}_{1}, \eta_{2} \in \mathbb{R}^{n}$ are the so-called historical terms, which take the following expressions:

$$
\begin{aligned}
& \boldsymbol{\eta}_{1}=\frac{1}{b_{0}} \sum_{j=1}^{s} b_{j} \mathbf{z}_{-j} \\
& \boldsymbol{\eta}_{2}=\frac{1}{b_{0}} \sum_{j=1}^{s} b_{j} \dot{\mathbf{z}}_{-j}
\end{aligned}
$$

where $\mathbf{z}_{-j}$ and $\dot{\mathbf{z}}_{-j}$ represent the previous $j$ th coordinates and velocities; $s$ represents the order of the integrator; and $b_{0}$ and $b_{j}$ are terms that depend on the order of the integrator. Additional details on these equations can be found in [13-15, 19].

Equation (2.9) constitutes a set of nonlinear equations that can be solved for the coordinates and velocities using, for instance, the Newton-Raphson method. 
The following Jacobian matrix can be defined to that effect:

$$
\mathbf{H}_{\mathbf{p}}=\left[\begin{array}{cccc}
\mathbf{F}_{\mathbf{z}} & \mathbf{F}_{\dot{\mathbf{z}}} & \mathbf{F}_{\ddot{\mathbf{z}}} & \mathbf{F}_{\boldsymbol{\lambda}} \\
\boldsymbol{\Phi}_{\mathbf{z}} & \mathbf{0} & \mathbf{0} & \mathbf{0} \\
\dot{\boldsymbol{\Phi}}_{\mathbf{z}} & \dot{\boldsymbol{\Phi}}_{\dot{\mathbf{z}}} & \mathbf{0} & \mathbf{0} \\
\ddot{\boldsymbol{\Phi}}_{\mathbf{z}} & \ddot{\boldsymbol{\Phi}}_{\dot{\mathbf{z}}} & \ddot{\boldsymbol{\Phi}}_{\ddot{\mathbf{z}}} & \mathbf{0} \\
-\mathbf{U}_{0}^{\mathrm{T}} & \beta_{0} \mathbf{U}_{0}^{\mathrm{T}} & \mathbf{0} & \mathbf{0} \\
\mathbf{0} & -\mathbf{U}_{0}^{\mathrm{T}} & \beta_{0} \mathbf{U}_{0}^{\mathrm{T}} & \mathbf{0}
\end{array}\right]
$$

where $\beta_{0}$ is an integration parameter that depends on the order of the integrator.

Equation (2.12) plays a critical role in the solution of the nonlinear equations. Certain steps can be taken to reduce its large size; specifically, we apply the method presented in [42], which consists of applying a velocity transformation $\mathbf{R}_{\mathrm{z}} \in \mathbb{R}^{n \times f}$ that maps the independent velocities into the dependent ones. This requires the partitioning of coordinates into independent $\left(\mathbf{z}^{\mathrm{i}} \in \mathbb{R}^{f}\right)$ and dependent $\left(\mathbf{z}^{\mathrm{d}} \in \mathbb{R}^{m}\right)$ sets. By doing so, the Lagrange multipliers can be eliminated from the dynamic equations and the Jacobian matrix. Algebraic manipulation of Equations (2.9) and (2.12) gives the increments within the Newton-Raphson iteration:

$$
\begin{aligned}
\Delta \mathbf{z}^{\mathrm{i}} & =\left(\mathbf{F}_{\ddot{\mathbf{z}}}^{\mathrm{i} \rho}-\beta_{0} \mathbf{F}_{\dot{\mathbf{z}}}^{\mathrm{i} \rho}+\beta_{0}^{2} \mathbf{F}_{\mathbf{z}}^{\rho}\right)^{-1} \beta_{0}^{2} \mathbf{R}_{\mathrm{z}}^{\mathrm{T}} \mathbf{F} \\
\Delta \mathbf{z}^{\mathrm{d}} & =-\left(\boldsymbol{\Phi}_{\mathbf{z}}^{\mathrm{d}}\right)^{-1}\left(\boldsymbol{\Phi}_{\mathbf{z}}^{\mathrm{i}} \Delta \mathbf{z}^{\mathrm{i}}-\boldsymbol{\Phi}\right) \\
\Delta \dot{\mathbf{z}}^{\mathrm{i}} & =\frac{1}{\beta_{0}} \Delta \mathbf{z}^{\mathrm{i}} \\
\Delta \dot{\mathbf{z}}^{\mathrm{d}} & =-\left(\boldsymbol{\Phi}_{\mathbf{z}}^{\mathrm{d}}\right)^{-1}\left(\boldsymbol{\Phi}_{\mathbf{z}}^{\mathrm{i}} \Delta \dot{\mathbf{z}}^{\mathrm{i}}+\boldsymbol{\Phi}_{t}\right) \\
\Delta \ddot{\mathbf{z}}^{\mathrm{i}} & =\frac{1}{\beta_{0}^{2}} \mathbf{z}^{\mathrm{i}} \\
\Delta \ddot{\mathbf{z}}^{\mathrm{d}} & =-\left(\boldsymbol{\Phi}_{\mathbf{z}}^{\mathrm{d}}\right)^{-1}\left(\dot{\boldsymbol{\Phi}}_{\mathbf{z}}^{\mathrm{d}} \Delta \dot{\mathbf{z}}^{\mathrm{d}}+\dot{\boldsymbol{\Phi}}_{\mathbf{z}}^{\mathrm{i}} \Delta \dot{\mathbf{z}}^{\mathrm{i}}+\boldsymbol{\Phi}_{t t}\right)
\end{aligned}
$$

where the $\rho$ operator is defined for a generic matrix $\mathbf{G}$ as $\mathbf{G}^{\rho} \equiv \mathbf{R}_{\mathrm{Z}}^{\mathrm{T}} \mathbf{G R}_{\mathrm{z}}$, and $\boldsymbol{\Phi}_{t}$ and $\boldsymbol{\Phi}_{t t}$ represent the first and second partial derivatives of the constraints with 
respect to time (which are null if the constraint equations are scleronomic).

\subsubsection{Sets of bodies and derivatives}

An important aspect of this formulation is the definition and use of sets of bodies to represent the location of the bodies within the system, relative to other bodies $[16,91]$. Given a kinematic joint associated with relative coordinate $k$, the following sets of bodies can be defined: $\mathrm{I}(k)$ for the body whose inboard joint corresponds to coordinate $k$; II $(k)$ for bodies outboard of body I $(k)$; III $(k)$ for bodies between the base body and the I $(k)$ body; and IV $(k)$ for other bodies.

The purpose of defining these sets of bodies is to simplify the calculation of the Jacobian matrix. For instance, it can be shown that the derivative of $\dot{z}_{i}$ with respect to an outboard position $z_{j}$ is null, as only inboard bodies in the kinematic chain take part in the recursive calculation of kinematic magnitudes. In other words, outboard perturbations do not cause a variation in these magnitudes.

An example application in the context of forward recursive calculation is as follows. Given the equations of motion from which Lagrange multipliers have been eliminated,

$$
\mathbf{F} \equiv \mathbf{B}^{\mathrm{T}}(\mathbf{M} \dot{\mathbf{Y}}-\mathbf{Q})=\mathbf{0}
$$

by differentiating with respect to the relative coordinates and considering that the mass matrix is constant due to the use of local reference frames, the following holds:

$$
\mathbf{F}_{\mathbf{z}}=\mathbf{B}_{\mathbf{z}}^{\mathrm{T}}(\mathbf{M} \dot{\mathbf{Y}}-\mathbf{Q})+\mathbf{B}^{\mathrm{T}}\left(\mathbf{M} \dot{\mathbf{Y}}_{\mathbf{z}}-\mathbf{Q}_{\mathbf{z}}\right)
$$

In turn, to calculate this expression, it is necessary to calculate the derivative of 
the absolute accelerations with respect to $z_{k}$, based on Equation (2.4):

$$
\begin{aligned}
\left(\dot{\mathbf{Y}}_{i}\right)_{z_{k}} & =\left(\mathbf{B}_{i}\right)_{z_{k}} \dot{\mathbf{Y}}_{i-1}+\mathbf{B}_{i}\left(\dot{\mathbf{Y}}_{i-1}\right)_{z_{k}} \\
& +\left(\dot{\mathbf{B}}_{i}\right)_{z_{k}} \mathbf{Y}_{i-1}+\dot{\mathbf{B}}_{i}\left(\mathbf{Y}_{i-1}\right)_{z_{k}}+\left(\mathbf{b}_{i}\right)_{z_{k}} \ddot{z}_{i} \\
\left(\mathbf{Y}_{i}\right)_{z_{k}} & =\left(\mathbf{B}_{i}\right)_{z_{k}} \mathbf{Y}_{i-1}+\mathbf{B}_{i}\left(\mathbf{Y}_{i-1}\right)_{z_{k}}+\left(\mathbf{b}_{i}\right)_{z_{k}} \dot{z}_{i}
\end{aligned}
$$

From this expression and previous considerations, it is obvious that, for cases $i \in \mathrm{II}(k) \cup \operatorname{IV}(k)$, these derivatives are null. For case $\mathrm{I}(k)$, necessarily $(i-1) \in$ $\operatorname{III}(k)$ and so $\left(\mathbf{Y}_{i-1}\right)_{z_{k}}=\mathbf{0}$. For case $i \in \operatorname{III}(k)$, given the structure of $\left(\mathbf{B}_{i}\right)_{z_{k}}$ and $\left(\mathbf{b}_{i}\right)_{z_{k}}$, these only depend on the relative coordinate between a body and the inboard body; in this case, since $i \neq k$, they are null:

$$
\begin{aligned}
& \left(\mathbf{Y}_{i}^{\mathrm{I}}\right)_{z_{k}}=\left(\mathbf{B}_{i}\right)_{z_{k}} \mathbf{Y}_{i-1}+\left(\mathbf{b}_{i}\right)_{z_{k}} \dot{z}_{i} \\
& \left(\mathbf{Y}_{i}^{\mathrm{III}}\right)_{z_{k}}=\mathbf{B}_{i}\left(\mathbf{Y}_{i-1}\right)_{z_{k}}
\end{aligned}
$$

Similar reasoning can be applied to backward recursive calculations that are used, for instance, to calculate the terms $\mathbf{B}_{\mathbf{z}}$ and $\mathbf{Q}_{\mathbf{z}}$, presented in Equation (2.20). Using this approach, it is possible to build a library of recursive expressions to calculate most terms in the Jacobian $\left(\mathbf{H}_{\mathbf{p}}\right)$.

\subsection{Subsystem synthesis formulation}

The subsystem synthesis method (SSM) was proposed by Kim et al. to efficiently simulate vehicles $[65,66,70]$. By taking advantage of their special system topology, the equations of motion can be broken down into several small sets of independent dynamic equations corresponding to the chassis and each of the suspension subsystems. We will hereby use coordinates and reference frames that are similar to those of the DS-SRF.

If one follows an index-1 approach by imposing the loop-closure Lagrange 
multipliers onto the open-loop equations in Equation (1.23) and appending the acceleration-level loop-closure constraints $\left(\ddot{\boldsymbol{\Phi}}=\boldsymbol{\Phi}_{\mathrm{z}} \ddot{\mathbf{z}}+\dot{\boldsymbol{\Phi}}_{\mathrm{z}} \dot{\mathbf{z}}=\mathbf{0}\right)$, a dependent coordinate form of the dynamic equations arises:

$$
\left[\begin{array}{cc}
\mathbf{R}_{\mathrm{d}}^{\mathrm{T}} \mathbf{M}^{\Sigma} \mathbf{R}_{\mathrm{d}} & \boldsymbol{\Phi}_{\mathbf{z}}^{\mathrm{T}} \\
\boldsymbol{\Phi}_{\mathbf{z}} & \mathbf{0}
\end{array}\right]\left\{\begin{array}{c}
\ddot{\mathbf{z}} \\
\boldsymbol{\lambda}
\end{array}\right\}=\left\{\begin{array}{c}
\mathbf{R}_{\mathrm{d}}^{\mathrm{T}}\left(\mathbf{Q}^{\Sigma}-\mathbf{P}^{\Sigma}\right) \\
-\dot{\boldsymbol{\Phi}}_{\mathbf{z}} \dot{\mathbf{z}}
\end{array}\right\}
$$

The SSM can therefore be implemented through the use of dependent and independent relative coordinates, depending on whether we optimize the structure of $\mathbf{R}_{\mathrm{d}}^{\mathrm{T}} \mathbf{M}^{\Sigma} \mathbf{R}_{\mathrm{d}}$ in Equation (2.25) (dependent coordinate form) or $\mathbf{R}_{\mathrm{z}}^{\mathrm{T}} \mathbf{R}_{\mathrm{d}}^{\mathrm{T}} \mathbf{M}^{\Sigma} \mathbf{R}_{\mathrm{d}} \mathbf{R}_{\mathrm{z}}$ in Equation (1.31) (independent coordinate form).

Let us consider an example vehicle that will be analyzed in Section 2.4. The structure of its generalized mass matrices is illustrated in Figure 2.2 (dependent) and Figure 2.3 (independent). The different squares and labels show the diagonal and off-diagonal submatrices. We can see that there are 34 dependent coordinates and 14 independent ones. Both matrices are relatively sparse. Upon inspection, we see that the 4 suspension subsystems are independent and are coupled to the chassis. Each front suspension subsystem comprises 9 dependent relative coordinates and 2 independent ones; each rear suspension subsystem contains 5 dependent relative coordinates and 2 independent ones; and the chassis frame comprises 6 independent relative coordinates.

The equations of motion of the system can be rewritten in a sparse matrix form based on Equations (2.25) or (1.31), corresponding to the dependent and independent coordinate forms:

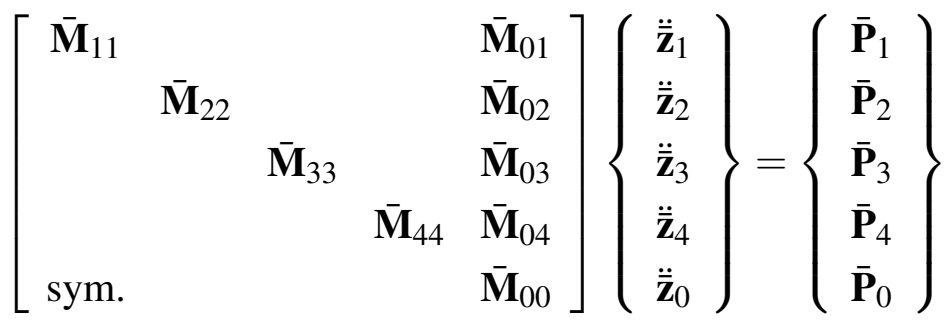




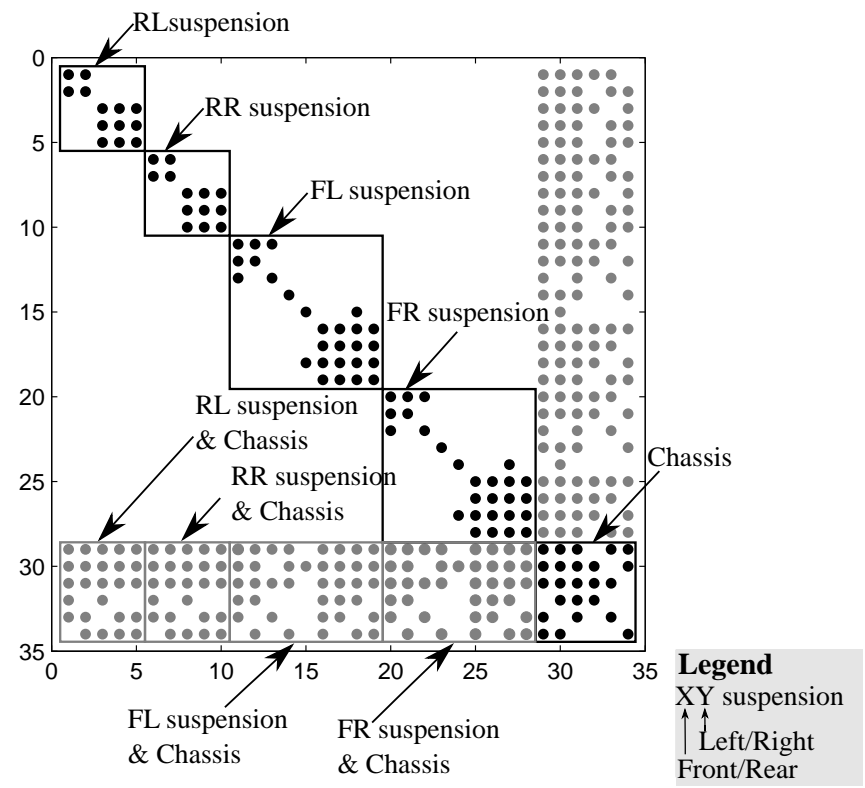

Figure 2.2: Structure of $\mathbf{R}_{\mathrm{d}}^{\mathrm{T}} \mathbf{M}^{\Sigma} \mathbf{R}_{\mathrm{d}}$ (dependent coordinate form)

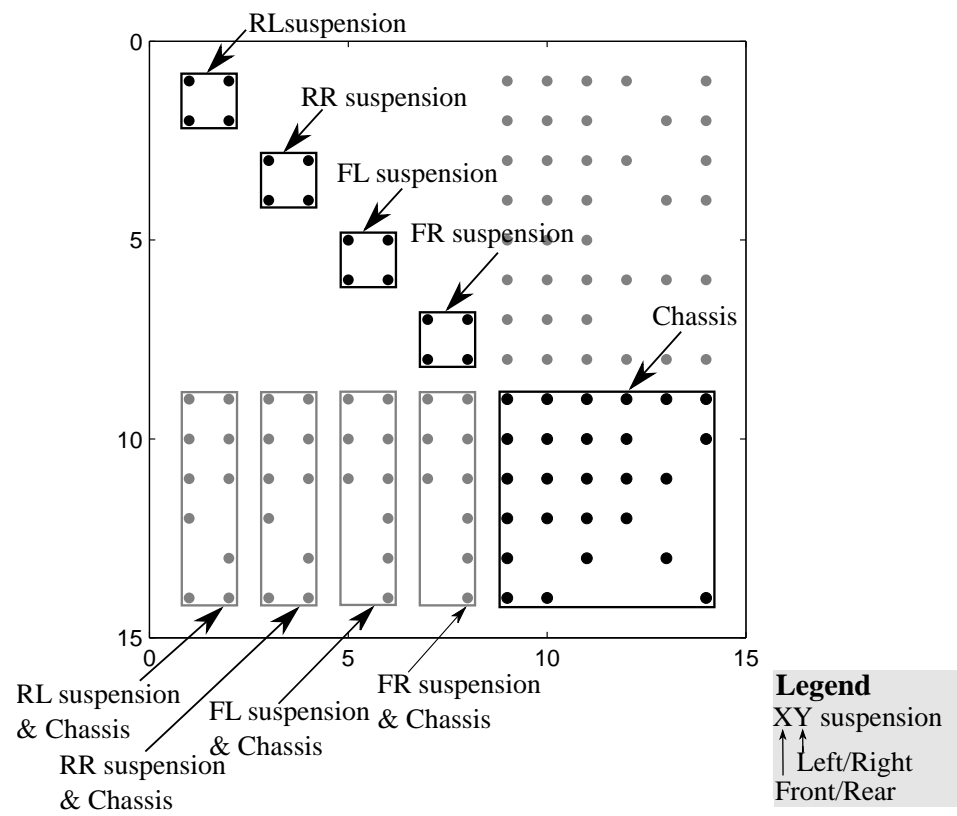

Figure 2.3: Structure of $\mathbf{R}_{\mathrm{z}}^{\mathrm{T}} \mathbf{R}_{\mathrm{d}}^{\mathrm{T}} \mathbf{M}^{\Sigma} \mathbf{R}_{\mathrm{d}} \mathbf{R}_{\mathrm{z}}$ (independent coordinate form) 
where terms $\overline{\mathbf{M}}_{i i}$ and $\overline{\mathbf{P}}_{i}(i=1,2,3,4)$ contain the generalized mass matrix and force vector of the $i$ th subsystem; terms $\overline{\mathbf{M}}_{0} \in \mathbb{R}^{6 \times 6}$ and $\overline{\mathbf{P}}_{0} \in \mathbb{R}^{6}$ contain the generalized mass matrix and force array of the chassis; matrix $\overline{\mathbf{M}}_{0 i}$ contains the inertia coupling matrix between the chassis and the $i$ th subsystem; vector $\ddot{\overline{\mathbf{z}}}_{i}$ contains the accelerations of the $i$ th subsystem; and vector $\ddot{\mathbf{z}}_{0} \in \mathbb{R}^{6}$ contains the accelerations of the chassis.

In the dependent coordinate form, terms $\overline{\mathbf{M}}_{i i}, \overline{\mathbf{P}}_{i}$, and $\ddot{\overline{\mathbf{z}}}_{i}$ take the following expressions:

$$
\begin{aligned}
\overline{\mathbf{M}}_{i i} & \equiv\left[\begin{array}{cc}
\left(\mathbf{R}_{\mathrm{d}}^{\mathrm{T}} \mathbf{M}^{\Sigma} \mathbf{R}_{\mathrm{d}}\right)_{i i} & \left(\boldsymbol{\Phi}_{\mathbf{z}}^{\mathrm{T}}\right)_{i} \\
\left(\boldsymbol{\Phi}_{\mathbf{z}}\right)_{i} & \mathbf{0}
\end{array}\right] \\
\overline{\mathbf{P}}_{i} & \equiv\left\{\begin{array}{c}
\mathbf{R}_{\mathrm{d}}^{\mathrm{T}}\left(\mathbf{Q}^{\Sigma}-\mathbf{P}^{\Sigma}\right) \\
-\dot{\boldsymbol{\Phi}}_{\mathbf{z}} \dot{\mathbf{z}}
\end{array}\right\}_{i} \\
\ddot{\mathbf{z}}_{i} & \equiv\left\{\begin{array}{c}
\ddot{\mathbf{z}} \\
\boldsymbol{\lambda}
\end{array}\right\}_{i}
\end{aligned}
$$

The size of Equation (2.26) is $(n+m)$ in the dependent case and $f$ in the independent one. Through mathematical manipulation, the SSM can be expressed as:

$$
\left(\overline{\mathbf{M}}_{0}+\sum_{i=1}^{n_{s}} \hat{\mathbf{M}}_{i}\right) \ddot{\overline{\mathbf{z}}}_{0}=\overline{\mathbf{P}}_{0}+\sum_{i=1}^{n_{s}} \hat{\mathbf{P}}_{i}
$$

where $n_{s}$ represents the number of subsystems (in the vehicle at hand, $n_{s}=4$ ). Terms $\hat{\mathbf{M}}_{i} \in \mathbb{R}^{6 \times 6}$ and $\hat{\mathbf{P}}_{i} \in \mathbb{R}^{6}$ represent the effective inertia matrix and force array of the $i$ th subsystem. They play a crucial role in the numerical integration, and can be compactly expressed as:

$$
\begin{aligned}
\hat{\mathbf{M}}_{i} & =-\overline{\mathbf{M}}_{0 i}^{\mathrm{T}} \overline{\mathbf{M}}_{i i}^{-1} \overline{\mathbf{M}}_{0 i}, \quad i=1,2,3,4 \\
\hat{\mathbf{P}}_{i} & =-\overline{\mathbf{M}}_{0 i}^{\mathrm{T}} \overline{\mathbf{M}}_{i i}^{-1} \overline{\mathbf{P}}_{i}, \quad i=1,2,3,4
\end{aligned}
$$

The size of Equation (2.30) equals the number of chassis accelerations, namely 
6 in both forms (pitch, roll, yaw and $X$-, $Y$-, and $Z$-positions). Once the accelerations of the chassis are determined, the suspension accelerations can be solved for through the following dynamic equations:

$$
\overline{\mathbf{M}}_{i i} \ddot{\mathbf{z}}_{i}=\overline{\mathbf{P}}_{i}-\overline{\mathbf{M}}_{0 i} \ddot{\mathbf{z}}_{0}, \quad i=1,2,3,4
$$

In the dependent coordinate form, the number of dynamic equations in Equation (2.33) is 15 per front suspension system $(n=9, m=6)$ and 8 per rear suspension system $(n=5, m=3)$. This means that the 52 system equations have been broken down into a 6-dimensional chassis equation, two 8-dimensional dynamic equations and two 15-dimensional dynamic equations.

In the independent coordinate case, the number of dynamic equations is $f=2$ for all subsystems. That is to say, the 14 system equations have been broken down into a 6-dimensional chassis equation and four 2-dimensional dynamic equations. The independent coordinate approach eliminates Lagrange multipliers and embeds loop-closure constraint equations, which leads to a more compact form.

\subsection{Results}

In order to carry out numerical comparisons in terms of solution accuracy and computational efficiency between the above formulations, a 28-DOF rover and a 16-DOF sedan vehicle are simulated. The multibody models are illustrated in Figures 2.4 and 2.5, respectively. The code has been written in $\mathrm{C} / \mathrm{C}++$ and run on an Intel Core i7 laptop with a $2.4 \mathrm{GHz}$ CPU, 8 GB of RAM and Microsoft Windows.

The rover can be modeled as an open-loop multibody system, as shown in Figure 2.6. The tree structure starts at the base element (the ground), continues to the chassis, and then branches off to the rest of the subsystems. Five auxiliary 


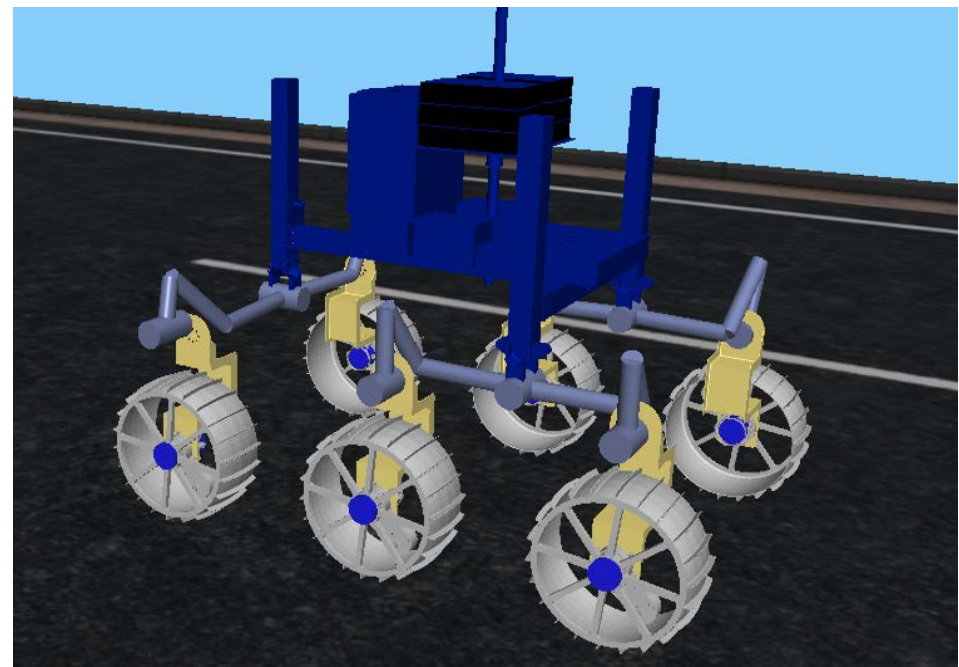

Figure 2.4: Exploration rover: multibody model

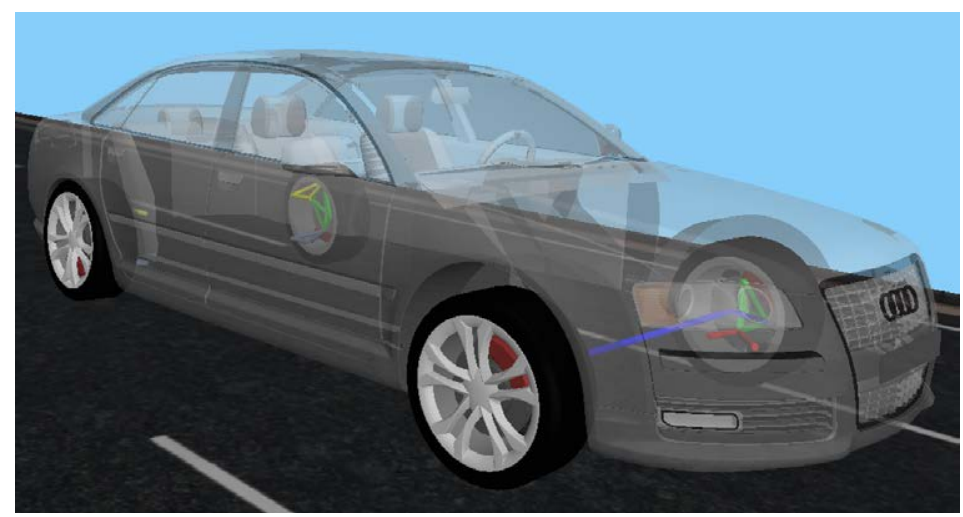

Figure 2.5: Sedan vehicle: multibody model 
massless bodies are introduced between the ground and the chassis to model its six DOFs using basic 1-DOF revolute and prismatic joints. The model contains three subsystems, namely the starboard, port and rear subsystems, which are independent of each other but are coupled with the chassis. The number of bodies, joints, and additional information are shown in Table 2.1.

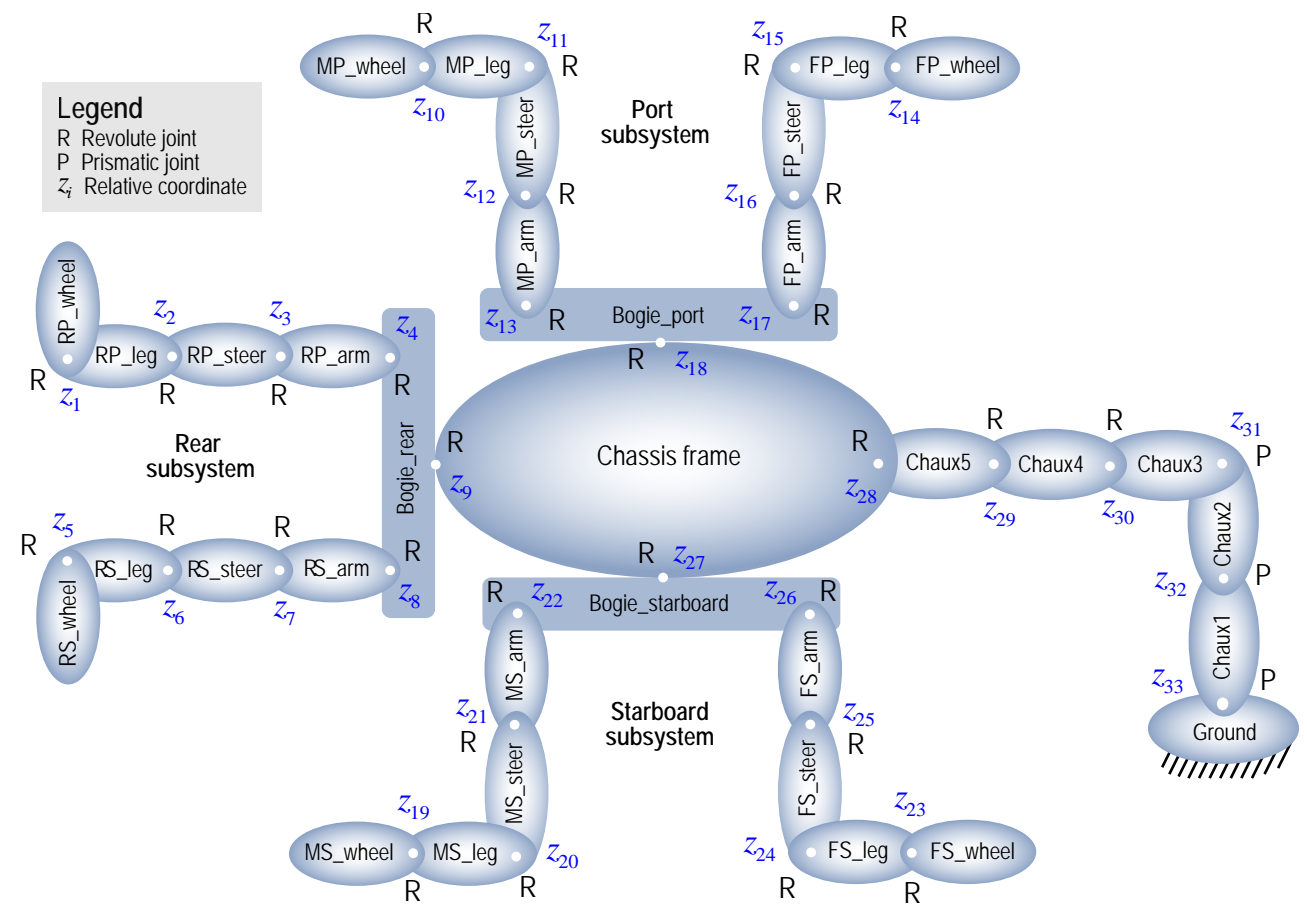

Figure 2.6: Rover: system topology

The sedan vehicle model contains MacPherson strut and double wishbone suspension systems in the front and rear axles, respectively. Tire forces are modeled using Pacejka's magic formula [83]. Figure 2.7 shows the tree topology of this model. Similar to the rover, several auxiliary massless bodies are introduced to model spherical, universal and 6-DOF joints by means of 1-DOF revolute and prismatic joints. Certain spherical joints are cut to open the closed loops. Within the SSM, the steering coordinate is split in two relative coordinates acting on the front left and front right suspension systems. Further information is gathered in Table 2.1. 


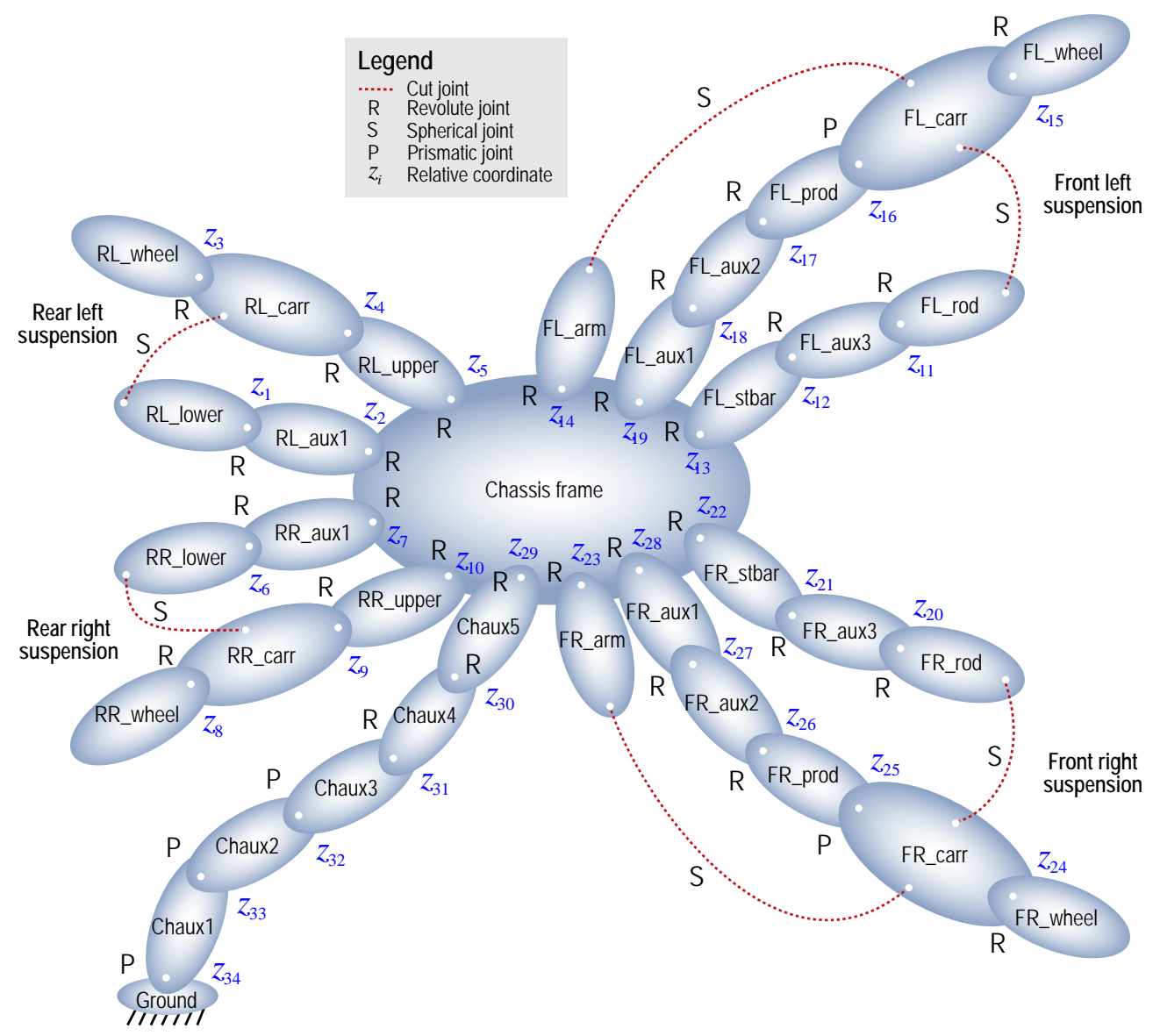

Figure 2.7: Sedan vehicle: system topology

\begin{tabular}{lcc}
\hline Item & Rover & Sedan \\
\hline Moving bodies (total) & 33 & 34 \\
Auxiliary bodies & 5 & 11 \\
Joints (including cut joints) & 34 & 40 \\
Cut joints & - & 6 \\
Subsystems & 3 & 4 \\
Dependent relative coordinates $(n)$ & - & 34 \\
Independent relative coordinates $(f)$ & 33 & 14 \\
Constraint equations $(m)$ & - & 18 \\
Guided coordinate & - & 2 \\
\hline
\end{tabular}

Table 2.1: Coordinate count 
In all simulations a slalom test maneuver along a flat road is performed. An explicit 4th-order Runge-Kutta integrator is employed in the explicit DS-SRF and the SSM, whereas backward differentiation formulas with a modified NewtonRaphson method are implemented in the implicit DS-SRF and the G-SRF. The sedan car is run with the SSM in both dependent- and independent-coordinate modes; this does not apply to the rover because all its coordinates are independent.

\subsubsection{Accuracy}

The explicit DS-SRF and the SSM should arrive at virtually identical solutions due to the similar formulations and same explicit integrators. On the other hand, local reference frames are used in the G-SRF, which generates different recursive velocity expressions and involves complicated coordinate transformation matrices.

The rover and sedan vehicles have been simulated for $1 \mathrm{~s}$ with different timesteps $(h)$. The maximum absolute difference in the position array $(\mathbf{z})$ between the DS-SRF and the other two formulations (SSM and G-SRF) are shown in Table 2.2. In the sedan vehicle part, the SSM corresponds to the independent coordinate form because the dependent form produced identical results. The errors increase with longer time-steps, and the largest error usually occurs at the end of the simulation because the differences build up over time.

In order to visualize these differences, the $X, Y$ and $Z$ translations of the chassis are plotted. The $h=1 \mathrm{~ms}$ responses are compared in Figures 2.8 and 2.9. The solutions from the explicit DS-SRF and the SSM are virtually identical, which agrees with the theory. The differences between the DS-SRF and the G-SRF are very small too, especially for short time-steps.

The maximum absolute difference between the G-SRF and the DS-SRF during the $h=2 \mathrm{~ms}$ rover and vehicle simulations are $1.65 \times 10^{-4} \mathrm{~mm}$ and $3.17 \times 10^{-4} \mathrm{~mm}$, respectively. Although longer time-steps in the implicit integrator yield a higher computational efficiency, the accuracy decreases sharply. Based on the accuracy 


\begin{tabular}{cccccc}
\hline \multirow{2}{*}{ Model } & $h$ & \multicolumn{2}{c}{ SSM } & \multicolumn{2}{c}{ G-SRF } \\
\cline { 3 - 6 } & $(\mathrm{ms})$ & $\max (|\Delta \mathbf{z}|)$ & $\operatorname{norm}(\Delta \mathbf{z})$ & $\max (|\Delta \mathbf{z}|)$ & $\operatorname{norm}(\Delta \mathbf{z})$ \\
\hline \multirow{4}{*}{ Rover } & 0.5 & $8.89 \times 10^{-15}$ & $3.34 \times 10^{-14}$ & $1.04 \times 10^{-5}$ & $1.86 \times 10^{-5}$ \\
& 1 & $8.88 \times 10^{-15}$ & $2.41 \times 10^{-14}$ & $4.13 \times 10^{-5}$ & $7.47 \times 10^{-5}$ \\
& 2 & $1.95 \times 10^{-14}$ & $3.08 \times 10^{-14}$ & $1.65 \times 10^{-4}$ & $3.13 \times 10^{-4}$ \\
\hline \multirow{4}{*}{ Sedan } & 0.5 & $1.45 \times 10^{-14}$ & $1.87 \times 10^{-14}$ & $5.28 \times 10^{-6}$ & $1.28 \times 10^{-5}$ \\
& 1 & $8.60 \times 10^{-15}$ & $1.38 \times 10^{-14}$ & $3.98 \times 10^{-5}$ & $9.61 \times 10^{-5}$ \\
& 2 & $2.74 \times 10^{-15}$ & $1.13 \times 10^{-14}$ & $3.17 \times 10^{-4}$ & $7.66 \times 10^{-4}$ \\
\hline
\end{tabular}

Table 2.2: Error incurred by the SSM and the G-SRF as compared with the DS-SRF
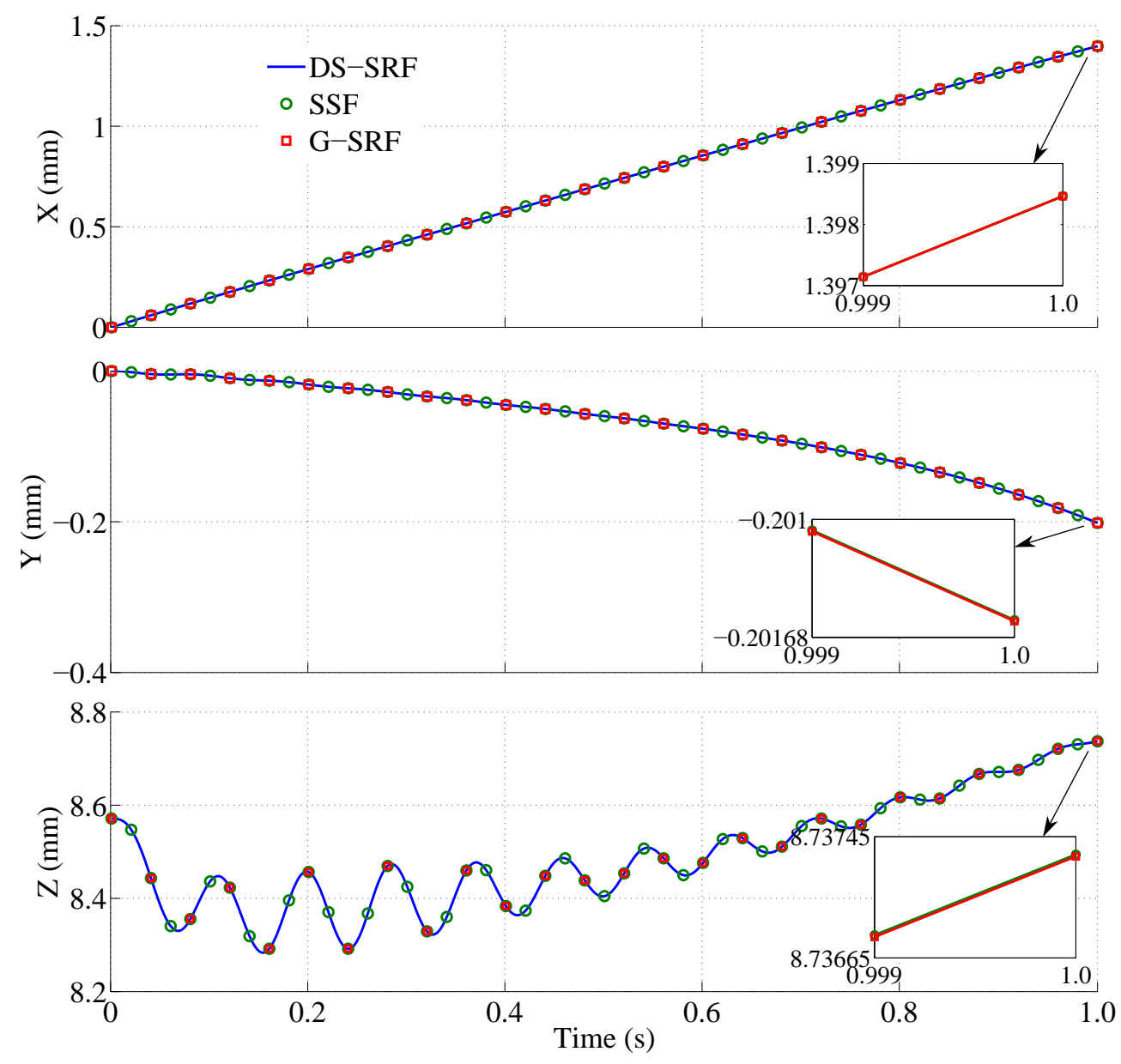

Figure 2.8: $X-, Y$ - and $Z$-displacements of the rover chassis 

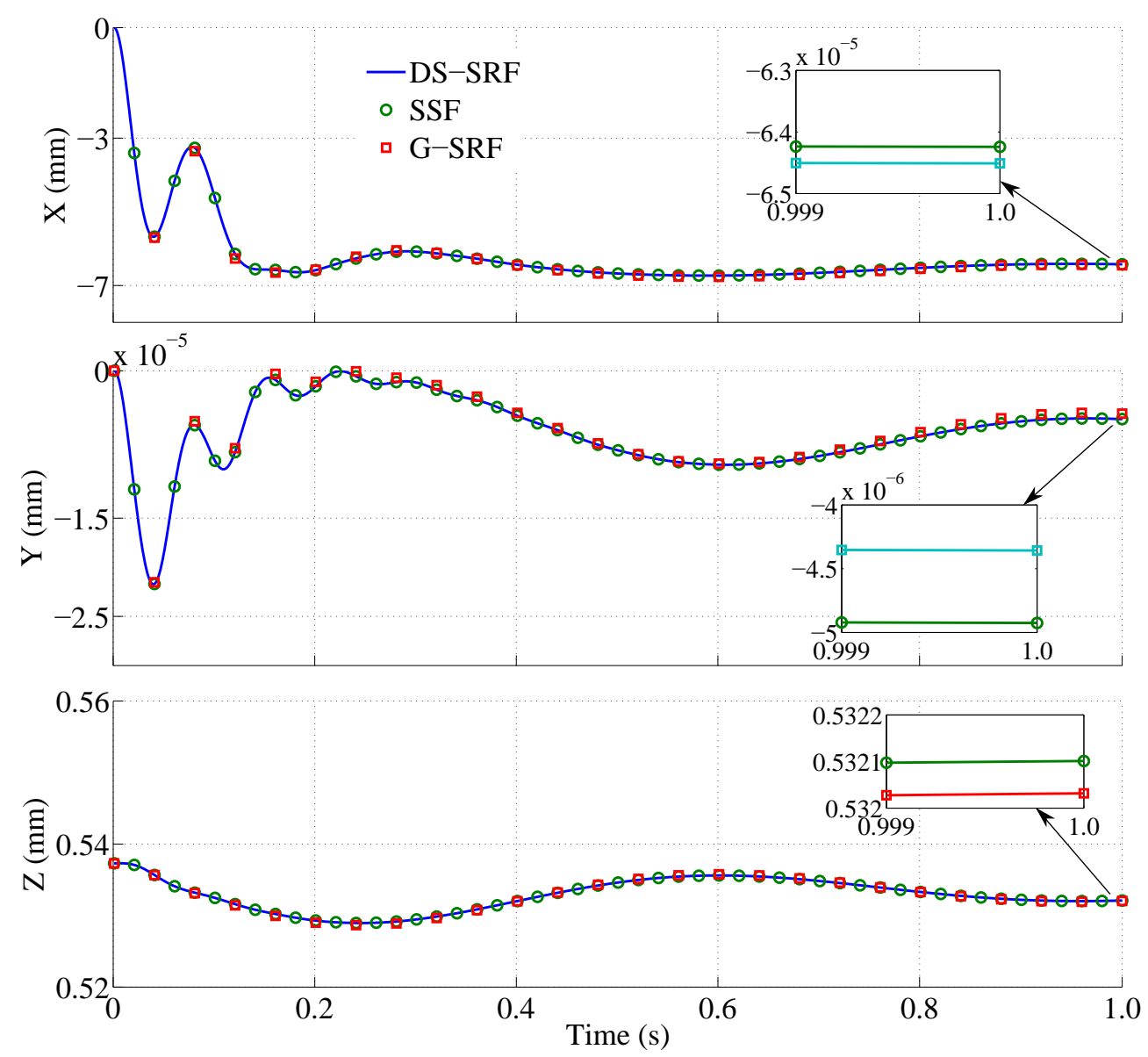

Figure 2.9: $X-, Y$ - and $Z$-displacements of the sedan chassis 
analysis in Table 2.2, the $h=2 \mathrm{~ms}$ implicit integrator solution is deemed equivalent to the $h=1 \mathrm{~ms}$ explicit Runge-Kutta one. Thus, in order to assess their computational efficiency, a $h=1 \mathrm{~ms}$ time-step will be used in the explicit DS-SRF and SSM, and a $h=2 \mathrm{~ms}$ time-step will be used in the implicit DS-SRF and G-SRF.

\subsubsection{Efficiency}

The rover and the sedan vehicle have been simulated for $10 \mathrm{~s}$ using the aforementioned formulations, and these simulations have been timed in detail. Note that Intel's Math Kernel Library has been used to solve the linear systems in Equations (2.13), (2.14), (2.16) and (2.18) within the G-SRF, and Equations (1.29) and (1.31) within the DS-SRF. On the other hand, Lapack libraries have been used to solve Equations (2.30)-(2.33) within the SSM.

Tables 2.3-2.5 contain information about the elapsed time (ET) of each significant formulation step or computer function (and their child functions) during the rover model simulation. The simulation is carried out with each of the presented methods: G-SRF, explicit/implicit DS-SRF and SSM. Analogously, Tables 2.6-2.8 show the ETs of the sedan vehicle model with each of the presented formulations: G-SRF, explicit/implicit DS-SRF and SSM in independent and dependent forms. A summary of all elapsed times is presented in Table 2.9.

According to the presented results, the DS-SRF is the most efficient formulation in both examples. The implicit DS-SRF is $21 \%$ faster than the explicit DS-SRF in the rover model, and $50 \%$ faster in the sedan vehicle. The SSM and the GSRF are considerably less efficient than the DS-SRF for both the rover and sedan models. Additionally, SSM's independent coordinate form is more efficient than the dependent coordinate one, namely $26 \%$ in the sedan vehicle example.

The computation of the Newton-Raphson derivatives constitutes, as expected, the critical step within the G-SRF, despite having employed differentiation techniques based on sets of bodies. The parallelization of such calculations would 


\begin{tabular}{lr}
\hline Algorithm step & ET \\
\hline $\mathbf{z}, \dot{\mathbf{z}}, \ddot{\mathbf{z}}-$ Equation (2.9) & $\mathbf{2 . 1 0} \mathbf{s}$ \\
\hline $\mathbf{F}_{\mathbf{z}}, \mathbf{F}_{\dot{\mathbf{z}}}, \mathbf{F}_{\ddot{\mathbf{z}}}-$ Eq. $(2.12)$, etc. & $45.7 \%$ \\
$\mathbf{Y}_{\mathbf{z}}, \mathbf{Y}_{\dot{\mathbf{z}}}, \dot{\mathbf{Y}}_{\mathbf{z}}, \dot{\mathbf{Y}}_{\dot{\mathbf{z}}}, \dot{\mathbf{Y}}_{\ddot{\mathbf{z}}}, \mathbf{Q}_{\mathbf{z}}, \mathbf{Q}_{\dot{\mathbf{z}}}$ & $38.8 \%$ \\
$\quad$ Eqs. (2.21), (2.22), etc. & \\
LU factorization & $6.9 \%$ \\
$\mathbf{Q}-$ User defined & $1.0 \%$ \\
Memory management & $1.0 \%$ \\
LU back substitution & $1.0 \%$ \\
Other & $<1.0 \%$ \\
\hline
\end{tabular}

Table 2.3: Rover simulation with the G-SRF

\begin{tabular}{lr}
\hline Explicit DS-SRF & ET \\
\hline$\ddot{\mathbf{z}}$ - Eq. (1.23) & $\mathbf{1 . 1 0 ~ s}$ \\
\hline $\mathbf{q}, \overline{\mathbf{M}}, \mathbf{M}^{\Sigma}$ - Eq. (1.24) & $34.5 \%$ \\
Cholesky decomp. & $29.3 \%$ \\
\multicolumn{1}{c}{ - Eq. (1.31) } & \\
$\mathbf{Q}^{\Sigma}$ - Eq. (1.25) & $12.9 \%$ \\
$\mathbf{Q}$ - User defined & $7.4 \%$ \\
$\mathbf{Z}$ - Eq. (1.20) & $3.9 \%$ \\
Cholesky back subst. & $1.7 \%$ \\
& \\
Memory management & $1.0 \%$ \\
Other Eq. $(1.31)$ & $<1.0 \%$ \\
\hline
\end{tabular}

\begin{tabular}{lr}
\hline Implicit DS-SRF & ET \\
\hline$\ddot{\mathbf{z}}$ - Eq. (1.23) & $\mathbf{0 . 8 7} \mathbf{~}$ \\
\hline Matrix product & $37.4 \%$ \\
LU factorization & $14.5 \%$ \\
$\mathbf{q}, \overline{\mathbf{M}}, \mathbf{M}^{\Sigma}$ - Eq. (1.24) & $14.3 \%$ \\
Memory management & $9.0 \%$ \\
$\mathbf{Z}$ - Eq. (1.20) & $7.1 \%$ \\
$\mathbf{Q}^{\Sigma}$ - Eq. (1.25) & $5.4 \%$ \\
$\mathbf{Q}$ - User defined & $3.6 \%$ \\
Matrix-vector product & $1.8 \%$ \\
LU back substitution & $1.7 \%$ \\
Other & $<1.0 \%$ \\
\hline
\end{tabular}

Table 2.4: Rover simulation with the DS-SRF 


\begin{tabular}{lr}
\hline Algorithm step & ET \\
\hline$\ddot{\mathbf{z}}$ - Eqs. (2.30), (2.33) & $\mathbf{2 . 4 0 ~ s}$ \\
\hline Matrix factorization & $15.5 \%$ \\
Solve linear equations & $15.2 \%$ \\
$\mathbf{q}, \overline{\mathbf{M}}, \mathbf{M}^{\Sigma}$ - Eq. (1.24) & $14.3 \%$ \\
$\overline{\mathbf{M}}_{i i}^{-1}$ - Eqs. (2.31), (2.32) & $12.8 \%$ \\
Memory management $^{\Sigma}$ & $10.8 \%$ \\
$\mathbf{Q}^{\Sigma}$ - Eq. (1.25) & $7.6 \%$ \\
$\mathbf{Z}$ - Eq. (1.20) & $4.0 \%$ \\
Matrix product & $3.6 \%$ \\
Matrix-vector product & $3.2 \%$ \\
$\mathbf{Q}-$ User defined & $1.2 \%$ \\
Other & $<1.0 \%$ \\
\hline
\end{tabular}

Table 2.5: Rover simulation with the SSM

\begin{tabular}{lr}
\hline Algorithm step & ET \\
\hline $\mathbf{z}, \dot{\mathbf{z}}, \ddot{\mathbf{z}}-$ Eq. (2.9) & $\mathbf{3 . 2 0 ~ s}$ \\
\hline $\mathbf{F}_{\mathbf{z}}, \mathbf{F}_{\dot{\mathbf{z}}}, \mathbf{F}_{\ddot{\mathbf{z}}}-$ Eq. $(2.12)$, etc. & $41.2 \%$ \\
$\mathbf{Y}_{\mathbf{z}}, \mathbf{Y}_{\dot{\mathbf{z}}}, \dot{\mathbf{Y}}_{\mathbf{z}}, \dot{\mathbf{Y}}_{\dot{\mathbf{z}}}, \dot{\mathbf{Y}}_{\ddot{\mathbf{z}}}, \mathbf{Q}_{\mathbf{z}}, \mathbf{Q}_{\dot{\mathbf{z}}}$ & $31.9 \%$ \\
$\quad$ - Eqs. $(2.21),(2.22)$, etc. & \\
$\mathbf{Y}$ - Eq. (2.3) & $8.3 \%$ \\
Matrix product & $6.4 \%$ \\
Memory management & $1.6 \%$ \\
LU factorization & $1.4 \%$ \\
$\boldsymbol{\Phi}_{\mathbf{z}}-$ Eq. (1.27) & $1.0 \%$ \\
Matrix-vector product & $1.0 \%$ \\
$\mathbf{Q}-$ User defined & $1.0 \%$ \\
Other & $<1.0 \%$ \\
\hline
\end{tabular}

Table 2.6: Sedan vehicle simulation with the G-SRF 


\begin{tabular}{lr}
\hline Explicit DS-SRF & ET \\
\hline$\ddot{\mathbf{z}}^{\mathrm{i}}$ - Eq. (1.31) & $\mathbf{2 . 0 0 ~ s}$ \\
\hline Matrix product & $19.0 \%$ \\
$\mathbf{q}, \overline{\mathbf{M}}, \mathbf{M}^{\Sigma}$ - Eq. (1.24) & $16.3 \%$ \\
LU back substitution & $11.4 \%$ \\
$\mathbf{Z}$ - Eq. (1.20) & $11.0 \%$ \\
$\mathbf{Q}^{\Sigma}$ - Eq. (1.25) & $8.2 \%$ \\
Memory management & $6.3 \%$ \\
$\boldsymbol{\Phi}_{\mathbf{z}}$ - Eq. (1.27) & $5.1 \%$ \\
$\mathbf{Q}$ - User defined & $4.1 \%$ \\
LU factorization & $3.4 \%$ \\
Cholesky decomp. & $1.9 \%$ \\
& \\
$\mathbf{\Phi}_{\mathbf{z}} \dot{\mathbf{z}}$-Eq. (1.32) & $1.9 \%$ \\
Other & $<2.0 \%$ \\
\hline
\end{tabular}

\begin{tabular}{lr}
\hline Implicit DS-SRF & ET \\
\hline$\ddot{\mathbf{z}}^{\text {i }}$ - Eq. (1.31) & $\mathbf{0 . 9 9} \mathbf{~}$ \\
\hline Matrix product & $17.2 \%$ \\
$\mathbf{q}, \overline{\mathbf{M}}, \mathbf{M}^{\Sigma}$ - Eq. (1.24) & $15.8 \%$ \\
LU factorization & $12.7 \%$ \\
LU back substitution & $7.9 \%$ \\
$\mathbf{\Phi}_{\mathbf{z}}-$ Eq. (1.27) & $7.9 \%$ \\
$\dot{\boldsymbol{\Phi}}_{\mathbf{z}}-$ Eq. (2.18) & $7.9 \%$ \\
$\dot{\boldsymbol{\Phi}}_{\mathbf{z}} \dot{\mathbf{z}}-$ Eq. (1.32) & $7.9 \%$ \\
$\mathbf{Z}-$ Eq. (1.20) & $4.8 \%$ \\
Memory management & $3.4 \%$ \\
$\mathbf{Q}-$ User defined & $3.2 \%$ \\
$\mathbf{Q}^{\Sigma}$ - Eq. (1.25) & $1.6 \%$ \\
Other & $<1.0 \%$ \\
\hline
\end{tabular}

Table 2.7: Sedan vehicle simulation with the DS-SRF

\begin{tabular}{|c|c|c|c|}
\hline Independent form SSM & ET & Dependent form SSM & ET \\
\hline$\ddot{\mathbf{z}}^{\mathrm{i}}-$ Eqs. (2.30), (2.33) & $2.80 \mathrm{~s}$ & $\ddot{\mathbf{z}}, \boldsymbol{\lambda}-$ Eqs. (2.30), (2.33) & $3.80 \mathrm{~s}$ \\
\hline $\mathbf{q}, \overline{\mathbf{M}}, \mathbf{M}^{\Sigma}-$ Eq. (1.24) & $12.9 \%$ & Matrix factorization & $17.1 \%$ \\
\hline Matrix product & $12.5 \%$ & $\overline{\mathbf{M}}_{i i}^{-1}-$ Eqs. (2.31), (2.32) & $11.2 \%$ \\
\hline LU back substitution & $10.1 \%$ & Solve linear equations & $9.5 \%$ \\
\hline Memory management & $7.1 \%$ & $\mathbf{q}, \overline{\mathbf{M}}, \mathbf{M}^{\Sigma}-$ Eq. (1.24) & $9.2 \%$ \\
\hline $\boldsymbol{\Phi}_{\mathbf{z}}-$ Eq. (1.27) & $6.4 \%$ & Memory management & $7.2 \%$ \\
\hline $\mathbf{Z}-$ Eq. (1.20) & $5.3 \%$ & LU back substitution & $6.1 \%$ \\
\hline $\mathbf{Q}^{\Sigma}$ - Eq. (1.25) & $5.3 \%$ & $\boldsymbol{\Phi}_{\mathbf{z}}-$ Eq. (1.27) & $5.6 \%$ \\
\hline$\overline{\mathbf{M}}_{i i}^{-1}-$ Eqs. (2.31), (2.32) & $5.3 \%$ & Matrix product & $4.6 \%$ \\
\hline Solve linear equations & $4.9 \%$ & $\mathbf{Q}^{\Sigma}-$ Eq. (1.25) & $3.1 \%$ \\
\hline Matrix factorization & $4.6 \%$ & LU factorization & $2.8 \%$ \\
\hline LU factorization & $3.5 \%$ & $\mathbf{Z}-$ Eq. (1.20) & $2.8 \%$ \\
\hline $\mathbf{Q}$ - User defined & $2.8 \%$ & $\mathbf{Q}$ - User defined & $2.6 \%$ \\
\hline$\dot{\boldsymbol{\Phi}}_{\mathrm{z}} \dot{\mathbf{z}}-$ Eq. $(1.32)$ & $2.1 \%$ & $\dot{\Phi}_{\mathrm{z}} \dot{\mathbf{z}}-$ Eq. $(1.32)$ & $2.0 \%$ \\
\hline Matrix-vector product & $2.1 \%$ & Memory management & $1.0 \%$ \\
\hline Memory management & $1.1 \%$ & Matrix-vector product & $1.0 \%$ \\
\hline Other & $<1.0 \%$ & Other & $<1.0 \%$ \\
\hline
\end{tabular}

Table 2.8: Sedan vehicle simulation with the SSM 


\begin{tabular}{llcr}
\hline Model & Method & $h(\mathrm{~ms})$ & ET (s) \\
\hline \multirow{4}{*}{ Rover } & G-SRF & 2 & 2.10 \\
& Explicit DS-SRF & 1 & 1.10 \\
& Implicit DS-SRF & 2 & $\mathbf{0 . 8 7}$ \\
& SSM & 1 & 2.40 \\
\hline \multirow{5}{*}{ Sedan } & G-SRF & 2 & 3.20 \\
& Explicit DS-SRF & 1 & 2.00 \\
& Implicit DS-SRF & 2 & $\mathbf{0 . 9 9}$ \\
& Independent form SSM & 1 & 2.80 \\
& Dependent form SSM & 1 & 3.80 \\
\hline
\end{tabular}

Table 2.9: Summary of elapsed times

definitely be worth trying toward increased performance. Regarding the SSM, the abundance of small linear systems seems to be its main burden, even though optimized numerical methods were used and lead matrix factorizations were reused whenever it was possible. Alternative techniques for the solution of such linear systems, such as parallelization or even analytical solutions, might alleviate these steps.

\subsection{Discussion}

We have presented a comparison of three state-of-the-art formulations for the efficient simulation of medium to large multibody systems such as vehicles; namely, a generalized recursive formulation, a double-step semi-recursive formulation and a subsystem synthesis method. Besides the theoretical foundations and unified notation, a 28-DOF rover and a 16-DOF sedan vehicle have been simulated in order to carry out a comparative study in terms of accuracy and computational efficiency. Results show that the three formulations produce consistent solutions and well beyond real-time performance. We have found the generalized semi-recursive formulation to be quite stable thanks to its implicit nature. Nevertheless, it heavily 
relies on the manual or topological derivation of Jacobian matrix terms within the Newton-Raphson method, which can be cumbersome. On the other hand, the value of the double-step semi-recursive formulation lies in the easiness of implementation and robustness of the constraint enforcement technique, which result in the shortest computational times of all three formulations. Finally, the subsystem synthesis formulation constitutes an effective way of breaking down the system equations into smaller sets, and it is relatively easy to implement. However, it brought about longer computational times for the cases under study. Overall, a valuable comparison of three competitive topological formulations for the simulation of multibody systems has been presented. 


\section{Chapter 3}

\section{Efficient and accurate modeling of rigid rods}

The double-step semi-recursive formulation presented in Chapter 1.3 if the basis of this section. The reader is encouraged to review its basic expressions to better understand what follows. By taking advantage of the cut-joint and rod-removal techniques through a double-step velocity transformation, this formulation has proven to be remarkably efficient. The rod-removal technique is employed, primarily, to reduce the number of differential and constraint equations. As a result, inertia and external forces are applied to neighboring bodies. Those inertia forces depend on unknown accelerations, a fact that contributes to the complexity of the system inertia matrix. In search of performance improvement, this Chapter presents an approximation of rod-related inertia forces by using accelerations from previous time-steps. Additionally, a mass matrix partition is carried out to preserve the accuracy of the original formulation. In order to assess the computational efficiency and solution accuracy of the presented approach, a 15-DOF, 12-rod sedan vehicle model with MacPherson strut and multi-link suspension systems is modeled, simulated and analyzed. 


\subsection{Introduction}

Vehicle dynamics has been a relevant application of multibody systems (MBS) since the seventies. Standard road vehicles are very often considered as complex, closedchain MBS with at least 15-DOF, namely: 6 DOF in the chassis frame (pitch, roll and yaw angles and $X, Y$ and $Z$ translations), 8 DOF in the front and rear suspension systems to describe the vertical suspension displacements and the rotation of the wheels, and 1 DOF to control the steering system. Figure 3.1 shows the treediagram topology of a sedan vehicle model with MacPherson strut suspension in the front axle and five-bar multi-link suspension in the rear axle. Several auxiliary massless bodies have been introduced to model spherical, universal and free joints by means of 1-DOF revolute and prismatic joints [43]. Some additional details about the vehicle model depicted in Figure. 3.1 are gathered here. The number of bodies, joints, constraints and coordinates is shown in Table 3.1. The DOF count is detailed in Table 3.2. Finally, detailed descriptions of rod elements and cut joints are presented in Tables 3.3 and 3.4, respectively.

\begin{tabular}{lr}
\hline Item & Number \\
\hline Bodies (including auxiliary bodies) & 34 \\
Auxiliary bodies & 19 \\
Joints (including cut joints) & 35 \\
Dependent relative coordinates $(n)$ & 33 \\
Independent relative coordinates $(f)$ & 14 \\
Subsystems & 4 \\
Driving coordinate & 1 \\
Constraint equations $(m)$ & 18 \\
Eliminated rods & 12 \\
Eliminated joints & 2 \\
\hline
\end{tabular}

Table 3.1: Element count

The vehicle model shown in Figure 3.1 contains a number of rigid rods. A rigid rod (or simply rod) is hereby defined as a slender rigid body attached to the 


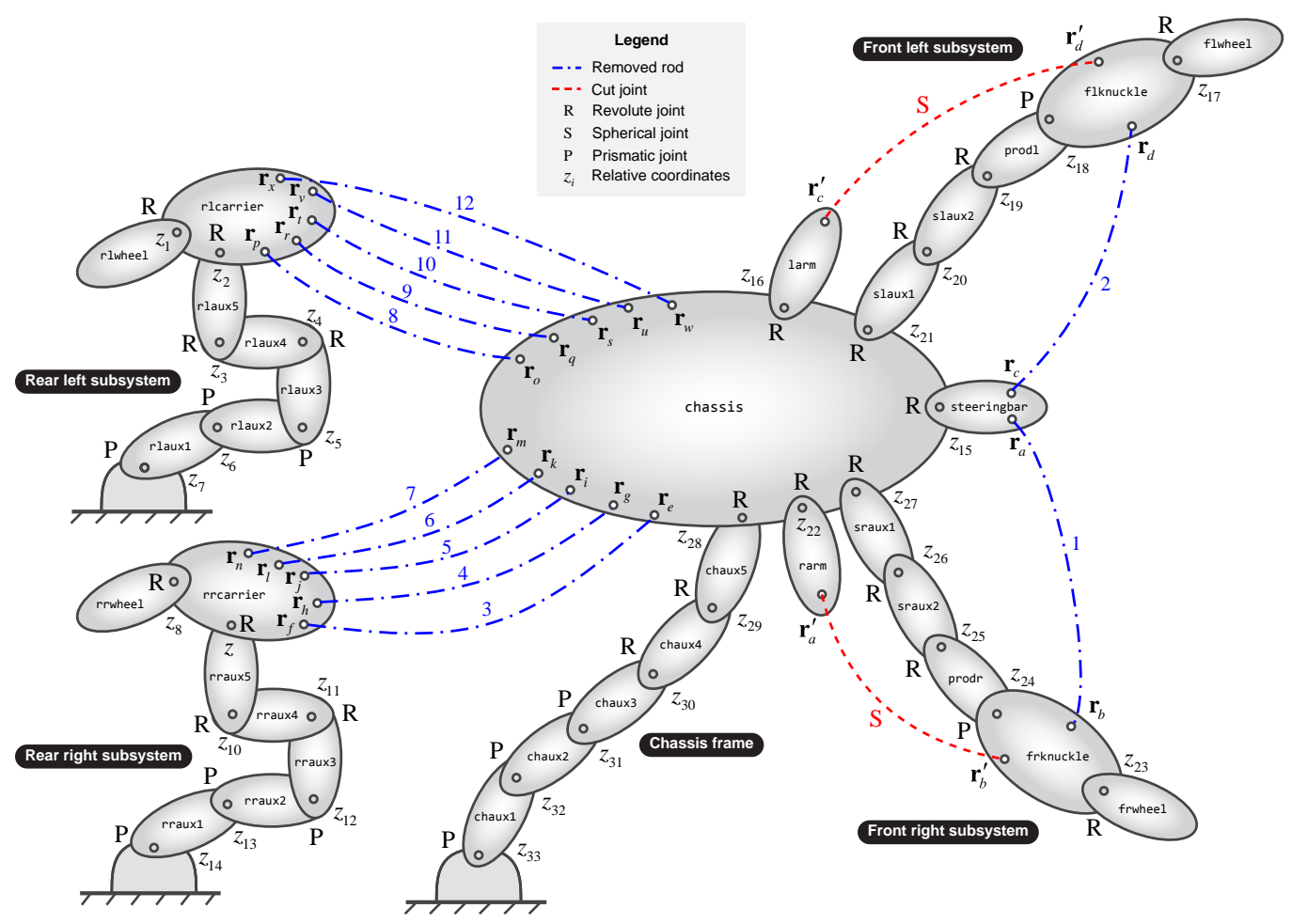

Figure 3.1: System topology of the 15-DOF sedan vehicle model

\begin{tabular}{lrrr}
\hline Item & Number & DOF/item & $\Sigma$ DOF \\
\hline Parts (no ground) & 33 & 6 & 198 \\
Prismatic joints & 12 & -5 & -60 \\
Revolute joints & 21 & -5 & -105 \\
Spherical joints & 2 & -3 & -6 \\
Removed rods & 12 & -1 & -12 \\
& & & Total DOF: 15
\end{tabular}

Table 3.2: Degree of freedom count 


\begin{tabular}{cccccc}
\hline ID & Mass(kg) & \multicolumn{2}{c}{ Connected bodies } & Connected points & Subsystem \\
\hline 1 & 2.0 & steeringbar & frknuckle & $\mathbf{r}_{a}, \mathbf{r}_{b}$ & FR \\
\hline 2 & 2.0 & steeringbar & flknuckle & $\mathbf{r}_{c}, \mathbf{r}_{d}$ & FL \\
\hline 3 & 2.0 & chassis & rrknuckle & $\mathbf{r}_{e}, \mathbf{r}_{f}$ & \\
4 & 2.0 & chassis & rrknuckle & $\mathbf{r}_{g}, \mathbf{r}_{h}$ & \\
5 & 4.0 & chassis & rrknuckle & $\mathbf{r}_{i}, \mathbf{r}_{j}$ & $\mathrm{RR}$ \\
6 & 1.0 & chassis & rrknuckle & $\mathbf{r}_{k}, \mathbf{r}_{l}$ & \\
7 & 3.0 & chassis & rrknuckle & $\mathbf{r}_{m}, \mathbf{r}_{n}$ & \\
\hline 8 & 2.0 & chassis & rlknuckle & $\mathbf{r}_{o}, \mathbf{r}_{p}$ & \\
9 & 2.0 & chassis & rlknuckle & $\mathbf{r}_{q}, \mathbf{r}_{r}$ & \\
10 & 4.0 & chassis & rlknuckle & $\mathbf{r}_{s}, \mathbf{r}_{t}$ & $\mathrm{RL}$ \\
11 & 1.0 & chassis & rlknuckle & $\mathbf{r}_{u}, \mathbf{r}_{v}$ & \\
12 & 3.0 & chassis & rlknuckle & $\mathbf{r}_{w}, \mathbf{r}_{x}$ & \\
\hline
\end{tabular}

Table 3.3: Rods

neighboring bodies through two spherical joints. It has a negligible moment of inertia around its centerline and a uniformly distributed mass. It can therefore be considered as a virtual, mathematical representation of a slender rigid link. In this context, it can be represented by using a constant-distance constraint and by applying its inertia properties_-including coupling terms - to the neighboring bodies.

\begin{tabular}{|c|c|c|c|c|c|}
\hline ID & Joint type & Conn & cted bodies & Connected points & Subsystem \\
\hline I & $\mathrm{S}$ & rarm & frknuckle & $\mathbf{r}_{a}^{\prime}, \mathbf{r}_{b}^{\prime}$ & FR \\
\hline II & $\mathrm{S}$ & larm & flknuckle & $\mathbf{r}_{c}^{\prime}, \mathbf{r}_{d}^{\prime}$ & $\mathrm{FL}$ \\
\hline
\end{tabular}

Table 3.4: Cut joints

Closed loops in the MBS have been temporarily opened by eliminating all rods and "cutting" certain spherical joints. Once the closed-loops have been opened, recursion techniques, together with the connectivity information, can be employed 
to formulate the open-loop equations of motion in a simple and efficient manner. This step involves a first velocity transformation between Cartesian and joint coordinates . Then, by imposing a minimal set of loop-closure constraint equations through Lagrange multipliers, the closed-loop equations of motion can be obtained [108]. The second velocity transformation is constructed in terms of the constraint equations corresponding to opening the closed loops, which are first expressed in natural or fully Cartesian coordinatesand then converted to relative coordinates. After the transformation, the state-space equations of motion of the entire system are expressed using a minimum set of independent coordinates. Regarding the removal or opening of rod loops, instead of cutting one of their end spherical joints (which equates to three constraint equations) and replacing the other one by a universal joint, rods are introduced as just one constant-distance constraint equation. This decreases the number of relative coordinates and constraint equations.

In this chapter, we present an improvement to the aforementioned independentcoordinate formulation. When a rod is eliminated in the context of the rod-removal technique, its dynamic properties, including second-derivative-based inertia forces (SDIFs), velocity-dependent inertia forces (VDIFs) and external forces, can be exactly considered. Both rod-related VDIFs and external forces can be directly added to the connecting bodies; this cannot be done, however, with rod-related SDIFs, because they are related to the unknown Cartesian accelerations. Usually one combines this term with the independent accelerations to solve the ordinary differential equations. As a consequence, the leading mass matrix no longer has a simple form for efficient computation. If, on the other hand, this unknown term is approximated by a known one and added to the (known) right-hand side forces, the mass matrix remains in simple form, and the computational efficiency is improved. Furthermore, the rod mass matrix can be partitioned into a diagonal matrix and a coupling matrix. The diagonal part is kept on the left-hand side of the equations of motion and the coupling matrix is moved to the right-hand side, which improves the solution accuracy without any loss in computational efficiency.

Together with the analysis of rod-related inertia forces and the methods to 
approximate them, the 15-DOF sedan car in Figure 3.1 is simulated to validate the algorithms and extract practical conclusions. Both accuracy and efficiency are assessed in the context of a general-purpose, MATLAB/C/C++ implementation.

\subsection{Double-step semi-recursive vehicle dynamics}

Let us consider, as an example, the topology of the vehicle model presented in Figure 3.1. Bodies have been numbered from the leaves to the root, so that children's numbers are less than their parents', as suggested by Negrut, Serban and Bae $[14,80,101]$. This prevents matrix fill-in in further steps. Bodies have the same identification numbers as their input joints (one per body). According to the system topology defined above, the first velocity transformation matrix in Equation (1.20) can be written as follows:

$\mathbf{R}=\left[\begin{array}{llllll}\mathbf{B}_{1}^{\Sigma} & \mathbf{0} & \mathbf{0} & \mathbf{0} & \mathbf{0} & \mathbf{0} \\ & \mathbf{B}_{2}^{\Sigma} & \mathbf{0} & \mathbf{0} & \mathbf{0} & \mathbf{0} \\ & & \mathbf{b}_{15} & \mathbf{0} & \mathbf{0} & \mathbf{B}_{\mathrm{CS}}^{\Sigma} \\ \vdots & & & \mathbf{B}_{3}^{\Sigma} & \mathbf{0} & \mathbf{B}_{\mathrm{CF}}^{\Sigma} \\ & . \cdot & & & \mathbf{B}_{4}^{\Sigma} & \mathbf{B}_{\mathrm{CF}}^{\Sigma} \\ \mathbf{0} & & \ldots & & & \mathbf{B}_{0}^{\Sigma}\end{array}\right] \equiv \mathbf{T R}_{\mathrm{d}}, \mathbf{B}_{0}^{\Sigma}=\left[\begin{array}{cccccc}\mathbf{b}_{28} & \mathbf{b}_{28} & \mathbf{b}_{28} & \mathbf{b}_{28} & \mathbf{b}_{28} & \mathbf{b}_{28} \\ & \mathbf{b}_{29} & \mathbf{b}_{29} & \mathbf{b}_{29} & \mathbf{b}_{29} & \mathbf{b}_{29} \\ & & \mathbf{b}_{30} & \mathbf{b}_{30} & \mathbf{b}_{30} & \mathbf{b}_{30} \\ \vdots & & & \mathbf{b}_{31} & \mathbf{b}_{31} & \mathbf{b}_{31} \\ & . \cdot & & & \mathbf{b}_{32} & \mathbf{b}_{32} \\ \mathbf{0} & & \ldots & & & \\ \mathbf{b}_{33}\end{array}\right]$

$\mathbf{B}_{1}^{\Sigma}=\left[\begin{array}{lllllll}\mathbf{b}_{1} & \mathbf{b}_{1} & \mathbf{b}_{1} & \mathbf{b}_{1} & \mathbf{b}_{1} & \mathbf{b}_{1} & \mathbf{b}_{1} \\ & \mathbf{b}_{2} & \mathbf{b}_{2} & \mathbf{b}_{2} & \mathbf{b}_{2} & \mathbf{b}_{2} & \mathbf{b}_{2} \\ & & \mathbf{b}_{3} & \mathbf{b}_{3} & \mathbf{b}_{3} & \mathbf{b}_{3} & \mathbf{b}_{3} \\ \vdots & & & \mathbf{b}_{4} & \mathbf{b}_{4} & \mathbf{b}_{4} & \mathbf{b}_{4} \\ & & . \cdot & & \mathbf{b}_{5} & \mathbf{b}_{5} & \mathbf{b}_{5} \\ & & & & & \mathbf{b}_{6} & \mathbf{b}_{6} \\ \mathbf{0} & & & \ldots & & & \mathbf{b}_{7}\end{array}\right], \mathbf{B}_{2}^{\Sigma}=\left[\begin{array}{lllllll}\mathbf{b}_{8} & \mathbf{b}_{8} & \mathbf{b}_{8} & \mathbf{b}_{8} & \mathbf{b}_{8} & \mathbf{b}_{8} & \mathbf{b}_{8} \\ & \mathbf{b}_{9} & \mathbf{b}_{9} & \mathbf{b}_{9} & \mathbf{b}_{9} & \mathbf{b}_{9} & \mathbf{b}_{9} \\ & & \mathbf{b}_{10} & \mathbf{b}_{10} & \mathbf{b}_{10} & \mathbf{b}_{10} & \mathbf{b}_{10} \\ \vdots & & & \mathbf{b}_{11} & \mathbf{b}_{11} & \mathbf{b}_{11} & \mathbf{b}_{11} \\ & & . \cdot & & \mathbf{b}_{12} & \mathbf{b}_{12} & \mathbf{b}_{12} \\ & & & & & \mathbf{b}_{13} & \mathbf{b}_{13} \\ \mathbf{0} & & & \ldots & & & \mathbf{b}_{14}\end{array}\right]$ 


$$
\mathbf{B}_{3}^{\Sigma}=\left[\begin{array}{llllll}
\mathbf{b}_{16} & \mathbf{0} & \mathbf{0} & \mathbf{0} & \mathbf{0} & \mathbf{0} \\
& \mathbf{b}_{17} & \mathbf{b}_{17} & \mathbf{b}_{17} & \mathbf{b}_{17} & \mathbf{b}_{17} \\
& & \mathbf{b}_{18} & \mathbf{b}_{18} & \mathbf{b}_{18} & \mathbf{b}_{18} \\
\vdots & & & \mathbf{b}_{19} & \mathbf{b}_{19} & \mathbf{b}_{19} \\
& . \cdot & & & \mathbf{b}_{20} & \mathbf{b}_{20} \\
\mathbf{0} & & \ldots & & & \mathbf{b}_{21}
\end{array}\right], \mathbf{B}_{4}^{\Sigma}=\left[\begin{array}{llllll}
\mathbf{b}_{22} & \mathbf{0} & \mathbf{0} & \mathbf{0} & \mathbf{0} & \mathbf{0} \\
& \mathbf{b}_{23} & \mathbf{b}_{23} & \mathbf{b}_{23} & \mathbf{b}_{23} & \mathbf{b}_{23} \\
& & \mathbf{b}_{24} & \mathbf{b}_{24} & \mathbf{b}_{24} & \mathbf{b}_{24} \\
\vdots & & & \mathbf{b}_{25} & \mathbf{b}_{25} & \mathbf{b}_{25} \\
& . \cdot & & & \mathbf{b}_{26} & \mathbf{b}_{26} \\
\mathbf{0} & & \ldots & & & \mathbf{b}_{27}
\end{array}\right]
$$

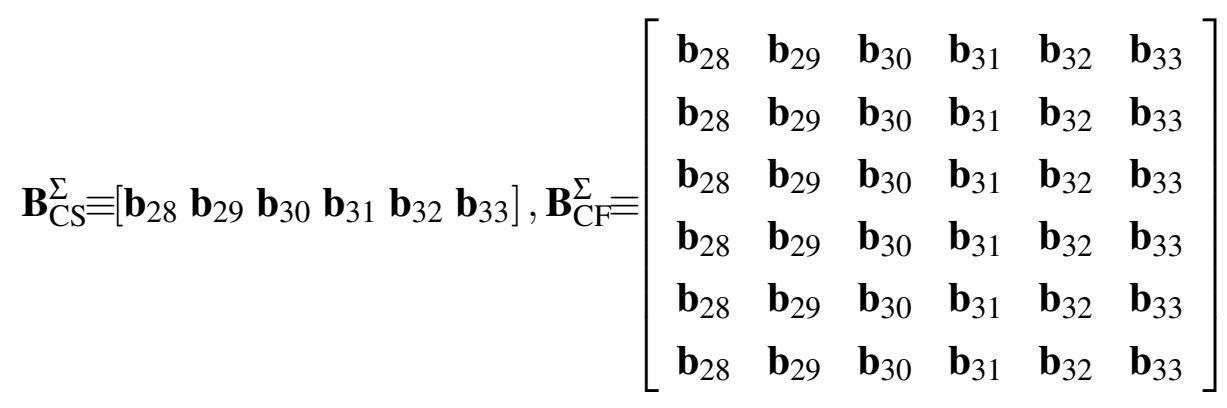

where matrix $\mathbf{T}$ is the path matrix, which represents the connectivity of the multibody system, and $\mathbf{R}_{\mathrm{d}}$ is a diagonal matrix whose elements are the $\mathbf{b}_{i}$ vectors defined in Equation (1.20). Submatrices $\mathbf{B}_{1}^{\Sigma}, \mathbf{B}_{2}^{\Sigma}, \mathbf{B}_{3}^{\Sigma}, \mathbf{B}_{4}^{\Sigma}$, and $\mathbf{B}_{0}^{\Sigma}$ are the elements of the first velocity transformation matrix corresponding to the rear left, rear right, front left and front right suspension systems and the chassis frame, respectively. Submatrices $\mathbf{B}_{\mathrm{CS}}^{\Sigma}$ and $\mathbf{B}_{\mathrm{CF}}^{\Sigma}$ correspond to the coupling between the chassis and the steering and front suspension systems, respectively.

\subsection{Rod-removal technique}

Rods are very often part of vehicle suspension systems. They not only constitute a numerical burden because of their numerous joint coordinates and contribution to the body count, but they also present numerical challenges associated with 
the neglected rotation around their axis [43]. Not uncommonly, rods are only considered as constant-distance constraints (thus neglecting the inertia forces associated with them) or are forbidden (for example requiring the replacement of one of the spherical joints by a universal joint). One of the main features of the presented formulation is the rod-removal technique and full consideration of their inertia properties [80], which makes it remarkably efficient and realistic. This technique, as already mentioned, is particularly attractive for vehicle models.

Applying the rod-removal technique to open the closed chains not only reduces the size of the dynamic equations, but also decreases the number of constraint equations. The reason is that removing one rod adds only one constant-distance constraint equation (recall Figure 1.2) and, in turn, eliminates one rigid body and two spherical joints. On the other hand, a cut spherical joint implies three constraint equations with no elimination of bodies, and a cut revolute joint implies five constraint equations with no elimination of bodies. Table 3.5 summarizes the effect of the different loop-closure methods on constraint equations and relative coordinates.

\begin{tabular}{ccccc}
\hline $\begin{array}{c}\text { Loop-closure } \\
\text { method }\end{array}$ & Constraints & $\begin{array}{c}\text { Eliminated } \\
\text { constraints }\end{array}$ & $\begin{array}{c}\text { Eliminated } \\
\text { bodies }\end{array}$ & $\begin{array}{c}\text { Eliminated } \\
\text { relative coordinates }\end{array}$ \\
\hline Rod & 1 & 2 & 1 & 6 \\
Spherical joint & 3 & 1 & 0 & 3 \\
Revolute joint & 5 & 1 & 0 & 1 \\
\hline
\end{tabular}

Table 3.5: Effect of cutting methods on constraints, bodies and relative coordinates

Let us look at the inertia and external forces that must be added to the neighboring bodies. According to [43], a rod connected to bodies $j$ and $k$ produces the 
following Cartesian forces:

$$
\begin{aligned}
& \left\{\begin{array}{l}
\overline{\mathbf{Q}}_{j} \\
\overline{\mathbf{Q}}_{k}
\end{array}\right\}=-\left[\begin{array}{ll}
\overline{\mathbf{M}}_{j j} & \overline{\mathbf{M}}_{j k} \\
\overline{\mathbf{M}}_{k j} & \overline{\mathbf{M}}_{k k}
\end{array}\right]\left\{\begin{array}{l}
\dot{\mathbf{Z}}_{j} \\
\dot{\mathbf{Z}}_{k}
\end{array}\right\} \\
& -\left\{\left[\begin{array}{c}
\mathbf{I}_{3} \\
\tilde{\mathbf{r}}_{j} \\
{\left[\begin{array}{c}
\mathbf{I}_{3} \\
\tilde{\mathbf{r}}_{k}
\end{array}\right]\left(\frac{m}{3} \tilde{\omega}_{j} \tilde{\omega}_{j} \mathbf{r}_{j}+\frac{m}{6} \tilde{\omega}_{k} \tilde{\omega}_{k} \tilde{\mathbf{r}}_{k}\right)} \\
\left.\tilde{\omega}_{j} \mathbf{r}_{j}+\frac{m}{3} \tilde{\omega}_{k} \tilde{\omega}_{k} \mathbf{r}_{k}\right)
\end{array}\right\}+\left\{\begin{array}{l}
\mathbf{f}_{j} \\
\tilde{\mathbf{r}}_{j} \mathbf{f}_{j} \\
\mathbf{f}_{k} \\
\tilde{\mathbf{r}}_{k} \mathbf{f}_{k}
\end{array}\right\}\right.
\end{aligned}
$$

in which,

$$
\overline{\mathbf{M}}_{j j} \equiv \frac{m}{3}\left[\begin{array}{cc}
\mathbf{I}_{3} & \tilde{\mathbf{r}}_{j}^{\mathrm{T}} \\
\tilde{\mathbf{r}}_{j} & \tilde{\mathbf{r}}_{j} \tilde{\mathbf{r}}_{j}^{\mathrm{T}}
\end{array}\right], \overline{\mathbf{M}}_{k k} \equiv \frac{m}{3}\left[\begin{array}{cc}
\mathbf{I}_{3} & \tilde{\mathbf{r}}_{k}^{\mathrm{T}} \\
\tilde{\mathbf{r}}_{k} & \tilde{\mathbf{r}}_{k} \tilde{\mathbf{r}}_{k}^{\mathrm{T}}
\end{array}\right], \overline{\mathbf{M}}_{j k} \equiv \overline{\mathbf{M}}_{k j}^{T} \equiv \frac{m}{6}\left[\begin{array}{cc}
\mathbf{I}_{3} & \tilde{\mathbf{r}}_{k}^{\mathrm{T}} \\
\tilde{\mathbf{r}}_{j} & \tilde{\mathbf{r}}_{j} \tilde{\mathbf{r}}_{k}^{\mathrm{T}}
\end{array}\right]
$$

where $m$ is the mass of the $\operatorname{rod}, \overline{\mathbf{Q}}_{j}$ and $\overline{\mathbf{Q}}_{k}$ are the forces propagated to the neighboring bodies through points $j$ and $k$, and $\mathbf{f}_{j}$ and $\mathbf{f}_{k}$ are the external forces applied on points $j$ and $k$ of the rod. Note that these force terms are expressed with respect to the previously defined global reference point. The upper tilde represents the skew-symmetric matrix associated with the cross product operator. Equation (3.5) is expressed more compactly as:

$$
\left\{\begin{array}{c}
\overline{\mathbf{Q}}_{j} \\
\overline{\mathbf{Q}}_{k}
\end{array}\right\}=-\left[\begin{array}{cc}
\overline{\mathbf{M}}_{j j} & \overline{\mathbf{M}}_{j k} \\
\overline{\mathbf{M}}_{k j} & \overline{\mathbf{M}}_{k k}
\end{array}\right]\left\{\begin{array}{c}
\dot{\mathbf{Z}}_{j} \\
\dot{\mathbf{Z}}_{k}
\end{array}\right\}-\left\{\begin{array}{c}
\overline{\mathbf{P}}_{j} \\
\overline{\mathbf{P}}_{k}
\end{array}\right\}+\left\{\begin{array}{c}
\overline{\mathbf{Q}}_{j} \\
\overline{\mathbf{Q}}_{k}
\end{array}\right\}
$$

The three terms on the right-hand side of Equations (3.5) and (3.7) are the rodrelated second-derivative-based inertia forces (SDIFs), velocity-dependent inertia forces (VDIFs) and external forces. The rod-related VDIFs and external forces can be directly added to the corresponding neighboring body forces. On the other hand, the rod-related SDIFs are unknown, as they couple the neighboring Cartesian accelerations via the mass matrices of the rods. Usually this term would be added to the left-hand side of the dynamic equations and solved with the rest of the unknown accelerations. Let us update the open-loop equations in Equation (1.22) 
with the new terms:

$$
\mathbf{R}_{\mathrm{d}}^{\mathrm{T}} \mathbf{T}^{\mathrm{T}} \overline{\mathbf{M}} \mathbf{T} \mathbf{R}_{\mathrm{d}} \ddot{\mathbf{z}}+\mathbf{R}_{\mathrm{d}}^{\mathrm{T}} \mathbf{T}^{\mathrm{T}} \overline{\mathbf{M}}_{\text {rods }} \dot{\mathbf{Z}}_{\text {rods }}=\mathbf{R}_{\mathrm{d}}^{\mathrm{T}} \mathbf{T}^{\mathrm{T}}\left[\left(\overline{\mathbf{Q}}+\overline{\mathbf{Q}}_{\text {rods }}\right)-\left(\overline{\mathbf{P}}+\overline{\mathbf{P}}_{\text {rods }}\right)\right]
$$

where $\overline{\mathbf{M}}_{\text {rods }}, \overline{\mathbf{Q}}_{\text {rods }}$ and $\overline{\mathbf{P}}_{\text {rods }}$ are the contributions of the rods to the inertia matrix, the external forces and the VDIFs of the eliminated rods, respectively. In compact form:

$$
\mathbf{R}_{\mathrm{d}}^{\mathrm{T}} \mathbf{M}^{\Sigma} \mathbf{R}_{\mathrm{d}} \ddot{\mathbf{z}}+\mathbf{R}_{\mathrm{d}}^{\mathrm{T}} \tilde{\mathbf{F}}_{\text {rods }}^{\Sigma}=\mathbf{R}_{\mathrm{d}}^{\mathrm{T}}\left(\mathbf{Q}^{\Sigma}-\mathbf{P}^{\Sigma}\right)+\mathbf{R}_{\mathrm{d}}^{\mathrm{T}}\left(\mathbf{Q}_{\text {rods }}^{\Sigma}-\mathbf{P}_{\text {rods }}^{\Sigma}\right)
$$

where $\tilde{\mathbf{F}}_{\text {rods }}^{\Sigma} \equiv \mathbf{T}^{\mathrm{T}} \overline{\mathbf{M}}_{\text {rods }} \dot{\mathbf{Z}}_{\text {rods }}, \mathbf{Q}_{\text {rods }}^{\Sigma} \equiv \mathbf{T}^{\mathrm{T}} \overline{\mathbf{Q}}_{\text {rods }}$ and $\mathbf{P}_{\text {rods }}^{\Sigma} \equiv \mathbf{T}^{\mathrm{T}} \overline{\mathbf{P}}_{\text {rods }}$ are the accumulated rod-related SDIFs, external forces and VDIFs due to the presence of rods. Tables 3.6 and 3.7 summarize these contributions for the case of the vehicle model.

\begin{tabular}{lll}
\hline & $\begin{array}{l}\text { Rod-related external } \\
\text { forces }\left(\overline{\mathbf{Q}}_{\text {rods }}\right)\end{array}$ & $\begin{array}{l}\text { Rod-related velocity-dependent } \\
\text { inertia forces }\left(\overline{\mathbf{P}}_{\text {rods }}\right)\end{array}$ \\
\hline Rear left knuckle & $\overline{\mathbf{Q}}_{p}+\overline{\mathbf{Q}}_{r}+\overline{\mathbf{Q}}_{t}+\overline{\mathbf{Q}}_{v}+\overline{\mathbf{Q}}_{s}$ & $\overline{\mathbf{P}}_{p}+\overline{\mathbf{P}}_{r}+\overline{\mathbf{P}}_{t}+\overline{\mathbf{P}}_{v}+\overline{\mathbf{P}}_{s}$ \\
Rear right knuckle & $\overline{\mathbf{Q}}_{f}+\overline{\mathbf{Q}}_{h}+\overline{\mathbf{Q}}_{j}+\overline{\mathbf{Q}}_{l}+\overline{\mathbf{Q}}_{n}$ & $\overline{\mathbf{P}}_{f}+\overline{\mathbf{P}}_{h}+\overline{\mathbf{P}}_{j}+\overline{\mathbf{P}}_{l}+\overline{\mathbf{P}}_{n}$ \\
Steering bar & $\overline{\mathbf{Q}}_{a}+\overline{\mathbf{Q}}_{c}$ & $\overline{\mathbf{P}}_{a}+\overline{\mathbf{P}}_{c}$ \\
Front left knuckle & $\overline{\mathbf{Q}}_{d}$ & $\overline{\mathbf{P}}_{d}$ \\
Front right knuckle & $\overline{\mathbf{Q}}_{b}$ & $\overline{\mathbf{P}}_{b}$ \\
& $\overline{\mathbf{Q}}_{e}+\overline{\mathbf{Q}}_{g}+\overline{\mathbf{Q}}_{i}+\overline{\mathbf{Q}}_{k}+\overline{\mathbf{Q}}_{m}+$ & $\overline{\mathbf{P}}_{e}+\overline{\mathbf{P}}_{g}+\overline{\mathbf{P}}_{i}+\overline{\mathbf{P}}_{k}+\overline{\mathbf{P}}_{m}+$ \\
Chassis frame & $\overline{\mathbf{Q}}_{o}+\overline{\mathbf{Q}}_{q}+\overline{\mathbf{Q}}_{s}+\overline{\mathbf{Q}}_{u}+\overline{\mathbf{Q}}_{w}$ & $\overline{\mathbf{P}}_{o}+\overline{\mathbf{P}}_{q}+\overline{\mathbf{P}}_{s}+\overline{\mathbf{P}}_{u}+\overline{\mathbf{P}}_{w}$ \\
& & \\
\hline
\end{tabular}

Table 3.6: Contribution of rods to the global external and inertia forces

By introducing Equation (1.21) into Equation (3.9), the rod-related SDIFs can be expressed in terms of relative accelerations. Then, by adding the corresponding rod-related VDIFs and external forces to the right-hand side of Equation (3.9), we arrive at the following set of open-loop equations in relative coordinates:

$$
\mathbf{R}_{\mathrm{d}}^{\mathrm{T}}\left(\mathbf{M}^{\Sigma}+\mathbf{M}_{\text {rods }}^{\Sigma}\right) \mathbf{R}_{\mathrm{d}} \ddot{\mathbf{z}}=\mathbf{R}_{\mathrm{d}}^{\mathrm{T}}\left[\left(\mathbf{Q}^{\Sigma}+\mathbf{Q}_{\text {rods }}^{\Sigma}\right)-\left(\mathbf{P}^{\Sigma}+\mathbf{P}_{\text {rods }}^{\Sigma}\right)-\mathbf{M}_{\text {rods }}^{\Sigma} \dot{\mathbf{R}}_{\mathrm{d}} \dot{\mathbf{z}}\right]
$$

where $\mathbf{M}_{\text {rods }}^{\Sigma} \equiv \mathbf{T}^{\mathrm{T}} \overline{\mathbf{M}}_{\text {rods }} \mathbf{T}$ is the accumulated inertia associated with the rods. 
As already mentioned, by taking into account the loop-closure constraints, a second velocity transformation $\left(\mathbf{R}_{\mathrm{z}}\right)$ can be computed to express the relative coordinates in terms of independent relative coordinates. We can then obtain the final set of motion differential equations in independent coordinates by introducing Equations (1.29) and (1.30):

$$
\begin{gathered}
\mathbf{R}_{\mathrm{z}}^{\mathrm{T}} \mathbf{R}_{\mathrm{d}}^{\mathrm{T}} \underbrace{\left(\mathbf{M}^{\Sigma}+\mathbf{M}_{\text {rods }}^{\Sigma}\right)}_{\hat{\mathbf{M}}^{\Sigma}} \mathbf{R}_{\mathrm{d}} \mathbf{R}_{\mathrm{z}} \ddot{\mathbf{z}}^{\mathrm{i}}= \\
\mathbf{R}_{\mathrm{z}}^{\mathrm{T}} \mathbf{R}_{\mathrm{d}}^{\mathrm{T}}[\underbrace{\left(\mathbf{Q}^{\Sigma}+\mathbf{Q}_{\text {rods }}^{\Sigma}\right)}_{\hat{\mathbf{Q}}^{\Sigma}}-\mathbf{T}^{\mathrm{T}} \underbrace{\left(\overline{\mathbf{M}}+\overline{\mathbf{M}}_{\text {rods }}\right)}_{\hat{\mathbf{M}}} \frac{d\left(\mathbf{T}_{\mathrm{d}} \mathbf{R}_{\mathrm{z}}\right)}{d t} \dot{\mathbf{z}}^{\mathrm{i}}-\mathbf{P}_{\text {rods }}^{\Sigma}]
\end{gathered}
$$

where $\hat{\mathbf{M}}^{\Sigma}, \hat{\mathbf{Q}}^{\Sigma}$ and $\hat{\mathbf{M}}$ are, respectively, the accumulated inertia matrix, the system

\begin{tabular}{|c|c|c|c|c|c|c|}
\hline & $\begin{array}{l}\text { Rear left } \\
\text { knuckle }\end{array}$ & $\begin{array}{l}\text { Rear right } \\
\text { knuckle }\end{array}$ & $\begin{array}{l}\text { Steering } \\
\text { bar }\end{array}$ & $\begin{array}{l}\text { Front left } \\
\text { knuckle }\end{array}$ & $\begin{array}{l}\text { Front right } \\
\text { knuckle }\end{array}$ & $\begin{array}{l}\text { Chassis } \\
\text { frame }\end{array}$ \\
\hline $\begin{array}{l}\text { Rear left } \\
\text { knuckle }\end{array}$ & $\begin{array}{l}\overline{\mathbf{M}}_{x x}+\overline{\mathbf{M}}_{r r}+ \\
\overline{\mathbf{M}}_{t t}+\overline{\mathbf{M}}_{v v}+ \\
\overline{\mathbf{M}}_{p p}\end{array}$ & & & & & sym. \\
\hline $\begin{array}{l}\text { Rear right } \\
\text { knuckle }\end{array}$ & & $\begin{array}{l}\overline{\mathbf{M}}_{n n}+\overline{\mathbf{M}}_{h h}+ \\
\overline{\mathbf{M}}_{j j}+\overline{\mathbf{M}}_{l l}+ \\
\overline{\mathbf{M}}_{f f}\end{array}$ & & & & \\
\hline $\begin{array}{l}\text { Steering } \\
\text { bar }\end{array}$ & & & $\begin{array}{l}\overline{\mathbf{M}}_{a a}+ \\
\overline{\mathbf{M}}_{c c}\end{array}$ & & & \\
\hline $\begin{array}{l}\text { Front left } \\
\text { knuckle }\end{array}$ & & & $\overline{\mathbf{M}}_{c d}$ & $\overline{\mathbf{M}}_{d d}$ & & \\
\hline $\begin{array}{l}\text { Front right } \\
\text { knuckle }\end{array}$ & & & $\overline{\mathbf{M}}_{a b}$ & & $\overline{\mathbf{M}}_{b b}$ & \\
\hline $\begin{array}{l}\text { Chassis } \\
\text { frame }\end{array}$ & $\begin{array}{l}\overline{\mathbf{M}}_{o p}+\overline{\mathbf{M}}_{q r}+ \\
\overline{\mathbf{M}}_{s t}+\overline{\mathbf{M}}_{u v}+ \\
\overline{\mathbf{M}}_{w x}\end{array}$ & $\begin{array}{l}\overline{\mathbf{M}}_{e f}+\overline{\mathbf{M}}_{g h}+ \\
\overline{\mathbf{M}}_{i j}+\overline{\mathbf{M}}_{k l}+ \\
\overline{\mathbf{M}}_{m n}\end{array}$ & & & & $\begin{array}{l}\overline{\mathbf{M}}_{e e}+\overline{\mathbf{M}}_{g g}+ \\
\overline{\mathbf{M}}_{m m}+\overline{\mathbf{M}}_{i i}+ \\
\overline{\mathbf{M}}_{k k}+\overline{\mathbf{M}}_{o o}+ \\
\overline{\mathbf{M}}_{q q}+\overline{\mathbf{M}}_{s s}+ \\
\overline{\mathbf{M}}_{u u}+\overline{\mathbf{M}}_{w w}\end{array}$ \\
\hline
\end{tabular}
external forces and the inertia matrix including the rods' contribution.

Table 3.7: Contribution of rods to the global inertia matrix $\left(\overline{\mathbf{M}}_{\text {rods }}\right)$ 


\subsection{Approximation of rod-related SDIFs}

The inclusion of rod terms adds complexity to the inertia matrices by filling them with the coupling terms shown in Table 3.6, as can be seen by comparing Equations (1.31) and (3.11). We will now examine the two velocity transformations to identify diagonal and off-diagonal terms. Specifically, let us analyze the openloop mass matrix $\mathbf{R}_{\mathrm{d}}^{\mathrm{T}} \hat{\mathbf{M}}^{\Sigma} \mathbf{R}_{\mathrm{d}}$ in Equation (3.11). The reason for not studying $\hat{\mathbf{M}}^{\Sigma}$ itself is that, for improved computational efficiency, we do not explicitly compute it in the computer code. Instead, we recursively compute $\mathbf{R}_{\mathrm{d}}^{\mathrm{T}} \hat{\mathbf{M}}^{\Sigma} \mathbf{R}_{\mathrm{d}}$ in a direct way. Figure 3.2 shows the sparsity pattern of matrix $\mathbf{R}_{\mathrm{d}}^{\mathrm{T}} \hat{\mathbf{M}}^{\Sigma} \mathbf{R}_{\mathrm{d}}$. The different squares and labels show the diagonal and off-diagonal submatrices. This matrix constitutes a sparse, symmetric matrix, and has the following properties in the vehicle example:

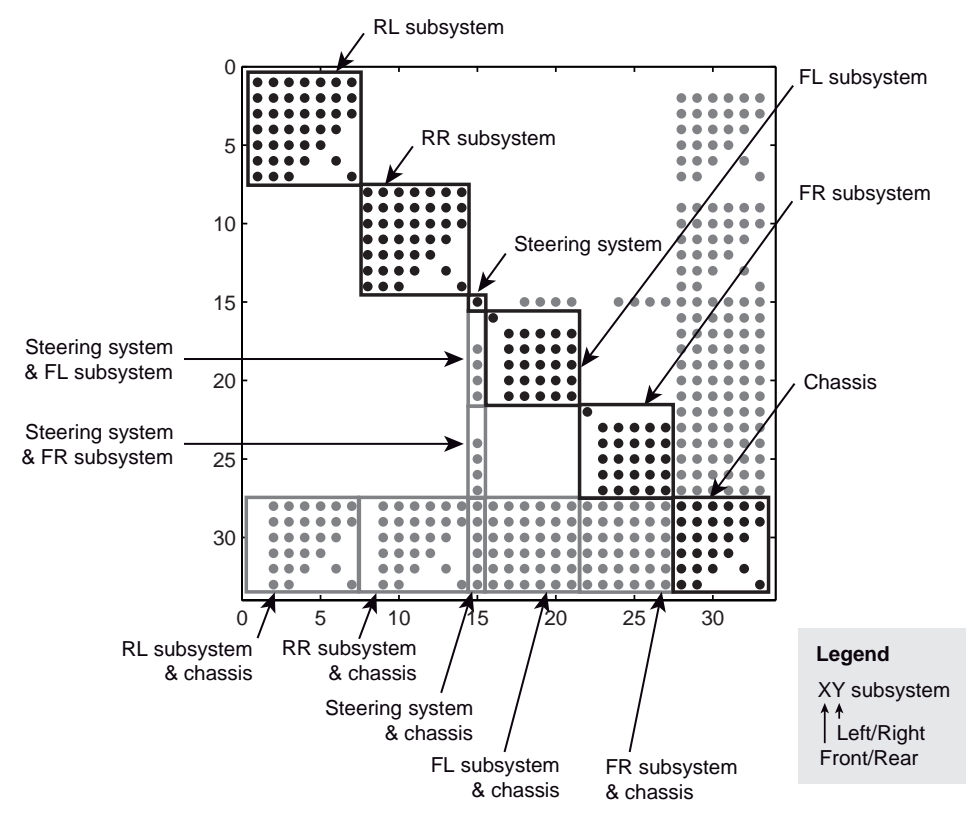

Figure 3.2: Structure of matrix $\mathbf{R}_{\mathrm{d}}^{\mathrm{T}} \hat{\mathbf{M}}^{\Sigma} \mathbf{R}_{\mathrm{d}}$ including rod terms

- The four suspension systems are independent of each other.

- The steering and rear suspension systems are independent of each other. 


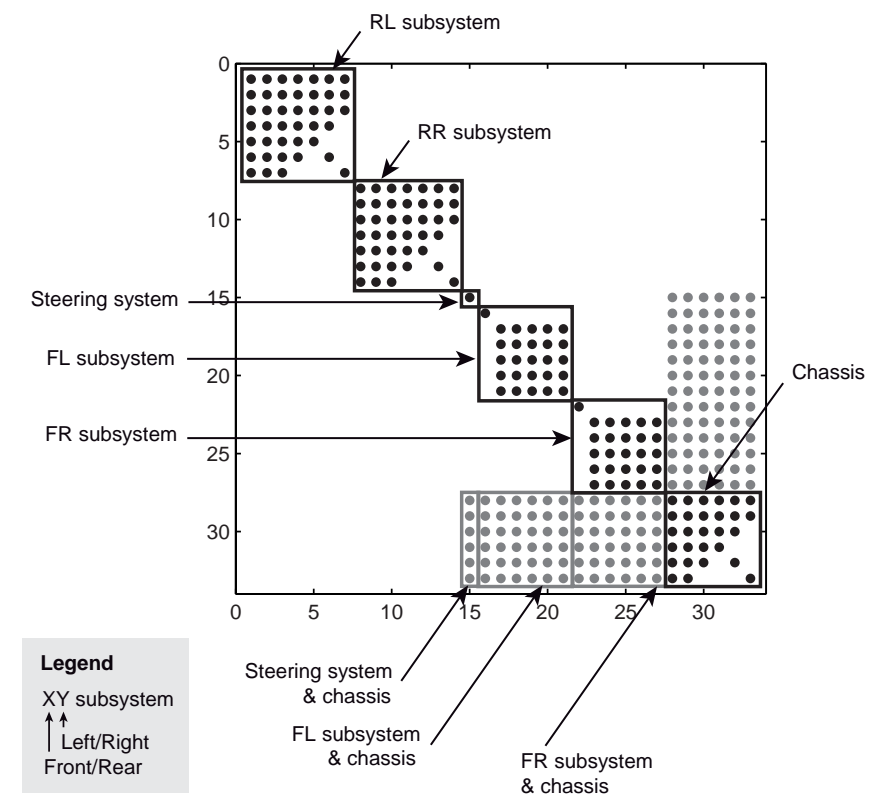

Figure 3.3: Structure of matrix $\mathbf{R}_{\mathrm{d}}^{\mathrm{T}} \mathbf{M}^{\Sigma} \mathbf{R}_{\mathrm{d}}$ with approximated rod-related SDIFs

- The steering and front suspension systems are coupled.

- The chassis frame is coupled with the suspension and steering systems.

- Each front and rear suspension subsystem is associated with six and seven relative coordinates, respectively.

- The steering system coordinate is known (predefined) and located at the center of the relative coordinate vector.

- There are six relative coordinates associated with the chassis frame.

Matrix $\mathbf{R}_{\mathrm{d}}^{\mathrm{T}} \hat{\mathbf{M}}^{\Sigma} \mathbf{R}_{\mathrm{d}}$ entails a high computational cost due to the accumulated coupling terms. A new way of taking into account the rods' inertia for enhanced efficiency is now explored. The approach is based on a simple yet effective idea: consider the accumulated rod-related SDIFs as known terms and move them to the right-hand side of the equations of motion. In this manner, the mass matrix is void of rod coupling terms, which results in improved performance. After 
approximating the rod Cartesian accelerations in Equation (3.9) we obtain the following expression:

$$
\mathbf{R}_{\mathrm{d}}^{\mathrm{T}} \mathbf{M}^{\Sigma} \mathbf{R}_{\mathrm{d}} \ddot{\mathbf{z}}=\mathbf{R}_{\mathrm{d}}^{\mathrm{T}}[\hat{\mathbf{Q}}^{\Sigma}-\underbrace{\left(\mathbf{P}^{\Sigma}+\mathbf{P}_{\text {rods }}^{\Sigma}\right)}_{\hat{\mathbf{P}}^{\Sigma}}]-\mathbf{R}_{\mathrm{d}}^{\mathrm{T}} \mathbf{T}^{\mathrm{T}} \overline{\mathbf{M}}_{\text {rods }} \hat{\mathbf{Z}}_{\text {rods }}
$$

where $\hat{\mathbf{P}}^{\Sigma}$ are the accumulated VDIFs including the rods' contribution. Vector $\hat{\mathbf{Z}}_{\text {rods }}$ is the approximate vector of rod-related Cartesian accelerations. In the vehicle model example this can be expressed as:

$$
\begin{aligned}
\hat{\mathbf{Z}}_{\text {rods }} \equiv & \left\{\mathbf{0}^{\mathrm{T}}, \hat{\mathbf{Z}}_{2}^{\mathrm{T}}, \mathbf{0}^{\mathrm{T}}, \ldots, \mathbf{0}^{\mathrm{T}}, \hat{\mathbf{\mathbf { Z }}}_{9}^{\mathrm{T}}, \mathbf{0}^{\mathrm{T}}, \ldots, \mathbf{0}^{\mathrm{T}}, \hat{\mathbf{Z}}_{15}^{\mathrm{T}}, \mathbf{0}^{\mathrm{T}},\right. \\
& \left.\mathbf{0}^{\mathrm{T}}, \hat{\mathbf{Z}}_{18}^{\mathrm{T}}, \mathbf{0}^{\mathrm{T}}, \ldots, \mathbf{0}^{\mathrm{T}}, \hat{\mathbf{Z}}_{24}^{\mathrm{T}}, \mathbf{0}^{\mathrm{T}}, \ldots, \mathbf{0}^{\mathrm{T}}, \hat{\mathbf{Z}}_{28}^{\mathrm{T}}, \mathbf{0}^{\mathrm{T}}, \ldots\right\}^{\mathrm{T}}
\end{aligned}
$$

Let us now look at the closed-loop equations, derived by applying the second velocity transformation $\left(\mathbf{R}_{\mathrm{z}}\right)$ and including the approximation of rod-related SDIFs:

$$
\mathbf{R}_{\mathrm{z}}^{\mathrm{T}} \mathbf{R}_{\mathrm{d}}^{\mathrm{T}} \mathbf{M}^{\Sigma} \mathbf{R}_{\mathrm{d}} \mathbf{R}_{\mathrm{z}} \ddot{\mathbf{z}}=\mathbf{R}_{\mathrm{z}}^{\mathrm{T}} \mathbf{R}_{\mathrm{d}}^{\mathrm{T}}\left(\hat{\mathbf{Q}}^{\Sigma}-\hat{\mathbf{P}}^{\Sigma}-\mathbf{M}^{\Sigma} \mathbf{R}_{\mathrm{d}} \dot{\mathbf{R}}_{\mathrm{z}} \dot{\mathbf{z}}^{\mathrm{i}}\right)-\mathbf{R}_{\mathrm{z}}^{\mathrm{T}} \mathbf{R}_{\mathrm{d}}^{\mathrm{T}} \mathbf{T}^{\mathrm{T}} \overline{\mathbf{M}}_{\text {rods }} \hat{\mathbf{Z}}_{\text {rods }}
$$

or, more compactly,

$$
\mathbf{R}_{\mathrm{z}}^{\mathrm{T}} \mathbf{R}_{\mathrm{d}}^{\mathrm{T}} \mathbf{M}^{\Sigma} \mathbf{R}_{\mathrm{d}} \mathbf{R}_{\mathrm{z}} \ddot{\mathbf{z}}^{\mathrm{i}}=\mathbf{R}_{\mathrm{z}}^{\mathrm{T}} \mathbf{R}_{\mathrm{d}}^{\mathrm{T}}\left(\hat{\mathbf{Q}}^{\Sigma}-\hat{\mathbf{P}}^{\Sigma}-\mathbf{M}^{\Sigma} \mathbf{R}_{\mathrm{d}} \dot{\mathbf{R}}_{\mathrm{z}} \dot{\mathbf{z}}^{\mathrm{i}}-\tilde{\mathbf{F}}_{\text {rods }}^{\Sigma}\right)
$$

By introducing Equation (1.30) and rearranging, Equation (3.15) can be rewritten as:

$$
\mathbf{R}_{\mathrm{z}}^{\mathrm{T}} \mathbf{R}_{\mathrm{d}}^{\mathrm{T}} \mathbf{M}^{\Sigma} \mathbf{R}_{\mathrm{d}} \mathbf{R}_{\mathrm{z}} \ddot{\mathbf{z}}^{\mathrm{i}}=\mathbf{R}_{\mathrm{z}}^{\mathrm{T}} \mathbf{R}_{\mathrm{d}}^{\mathrm{T}}[\hat{\mathbf{Q}}^{\Sigma}-\mathbf{T}^{\mathrm{T}} \overline{\mathbf{M}} \frac{d\left(\mathbf{T} \mathbf{R}_{\mathrm{d}} \mathbf{R}_{\mathrm{z}}\right)}{d t} \dot{\mathbf{z}}^{\mathrm{i}}-\underbrace{\left(\mathbf{P}_{\text {rods }}^{\Sigma}+\tilde{\mathbf{F}}_{\text {rods }}^{\Sigma}\right)}_{\mathbf{P}_{\text {rods }}^{\Sigma}}]
$$

which is the final form of the semi-recursive formulation following the rod-related SDIFs approximation approach. We can see, by comparing the left-hand side of Equations (3.11) and (3.16), that the approximation leads to a sparser mass matrix 
that has a narrower bandwidth. This means that the number of fills and operations in the factorization and solve steps associated with Equation (3.16) will be less. The structure of matrix $\mathbf{R}_{\mathrm{d}}^{\mathrm{T}} \mathbf{M}^{\Sigma} \mathbf{R}_{\mathrm{d}}$ is shown in Figure 3.3. The diagonal submatrices and the remaining coupling submatrices have the same form as those in Figure 3.2. We can extract some additional conclusions by comparing Figures 3.2 and 3.3:

- The chassis is still coupled with the front suspension and steering systems.

- The rear suspension systems and the chassis are no longer coupled.

- The steering system and the front suspension systems are no longer coupled either, since the steering rods connected to the front suspension systems are eliminated.

- The inertia and coupling matrices of the suspension and steering systems and the chassis are simpler than their Figure 3.2 counterparts, since the contribution of rods to inertia matrices is eliminated.

- The eliminated coupling matrices and inertia matrices are those shown in Table 3.3.

A preliminary benefit of this approach is that, due to the sparser structure of matrix $\mathbf{R}_{\mathrm{d}}^{\mathrm{T}} \mathbf{M}^{\Sigma} \mathbf{R}_{\mathrm{d}}$, the numerical integration can be carried out more efficiently [57]. However, we still have to address the evaluation of rod-related SDIF terms. One way of computing such terms is to extrapolate the accelerations based on previous (known) relative accelerations. A number of extrapolation methods are now presented.

The Cartesian accelerations and accumulated rod-related SDIFs shown in Equation (3.16) in the current integration time, $t$, can be expressed as:

$$
\begin{aligned}
& \hat{\ddot{\mathbf{z}}}_{t}=\mathbf{f}\left(\ddot{\mathbf{z}}_{t-h}, \ddot{\mathbf{z}}_{t-2 h}, \ldots\right) \\
& \hat{\mathbf{Z}}_{t}=\hat{\dot{\mathbf{Z}}}_{i-1}+\mathbf{b}_{i} \hat{\ddot{z}}_{i}+\mathbf{d}_{i} \\
& \tilde{\mathbf{F}}_{\text {rods }}^{\Sigma}=\mathbf{T}^{\mathrm{T}} \overline{\mathbf{M}}_{\text {rods }} \hat{\dot{\mathbf{Z}}}_{t, \text { rods }}
\end{aligned}
$$


where $h$ is the integration time-step and $\mathbf{f}$ is the extrapolation function. Three extrapolation methods of growing complexity have been explored: point approximation and linear and quadratic Lagrange extrapolation methods. These extrapolation methods are depicted in Figure 3.4. It can be seen how current relative accelerations are expressed in terms of relative accelerations from previous known integration steps. The curves represent the extrapolation polynomials and the points represent the current and previous acceleration values.

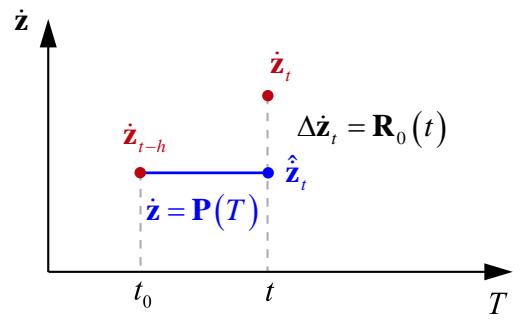

(a) Point approximation

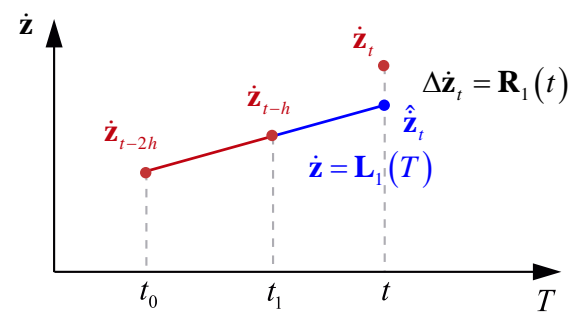

(b) Linear Lagrange extrapolation

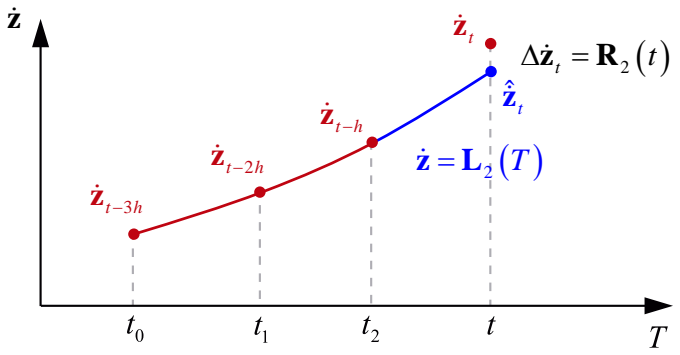

(c) Quadratic Lagrange extrapolation

Figure 3.4: Geometric description of extrapolation methods

Let us briefly describe the different extrapolation methods [27]. First, the current acceleration, $\hat{\ddot{\mathbf{z}}}_{t}$, according to the point approximation method (depicted in Figure 3.4(a)) can be written as:

$$
\begin{gathered}
\hat{\mathbf{z}}_{t}=\ddot{\mathbf{z}}_{t-h} \\
\mathbf{R}_{0}(t)=\mathbf{z}_{\xi}^{\mathrm{iii}}[t-(t-h)]=\mathbf{z}_{\xi}^{\mathrm{iii}} h=\mathrm{O}(h), \quad \xi \in(t-h, t)
\end{gathered}
$$

where $\mathbf{R}_{0}(t)$ is the corresponding remainder and Roman numerals indicate deriva- 
tives of order higher than two.

Second, the generic polynomial expression of the linear Lagrange extrapolation method (shown in Figure 3.4(b)) can be expressed as:

$$
\mathbf{L}_{1}(T)=\frac{T-t_{1}}{t_{0}-t_{1}} \ddot{\mathbf{z}}_{0}+\frac{T-t_{0}}{t_{1}-t_{0}} \ddot{\mathbf{z}}_{1}
$$

Substituting time $t$ and time-step $h$ we obtain the acceleration and remainder:

$$
\begin{gathered}
\hat{\mathbf{z}}_{t}=\mathbf{L}_{1}(t)=2 \ddot{\mathbf{z}}_{t-h}-\ddot{\mathbf{z}}_{t-2 h} \\
\mathbf{R}_{1}(t)=\mathbf{z}_{\xi}^{\mathrm{iv}}[t-(t-h)][t-(t-2 h)]=2 \mathbf{z}_{\xi}^{\mathrm{iv}} h^{2}=\mathrm{O}\left(h^{2}\right), \quad \xi \in(t-2 h, t)
\end{gathered}
$$

Third, the quadratic Lagrange extrapolation method (see Figure 3.4(c)) reads:

$$
\mathbf{L}_{2}(T)=\frac{\left(T-t_{1}\right)\left(T-t_{2}\right)}{\left(t_{0}-t_{1}\right)\left(t_{0}-t_{2}\right)} \ddot{\mathbf{z}}_{0}+\frac{\left(T-t_{0}\right)\left(T-t_{2}\right)}{\left(t_{1}-t_{0}\right)\left(t_{1}-t_{2}\right)} \ddot{\mathbf{z}}_{1}+\frac{\left(T-t_{0}\right)\left(T-t_{1}\right)}{\left(t_{2}-t_{0}\right)\left(t_{2}-t_{1}\right)} \ddot{\mathbf{z}}_{2}
$$

The acceleration and corresponding remainder can be obtained as:

$$
\begin{gathered}
\hat{\mathbf{z}}_{t}=\mathbf{L}_{2}(t)=3 \ddot{\mathbf{z}}_{t-h}-3 \ddot{\mathbf{z}}_{t-2 h}+\ddot{\mathbf{z}}_{t-3 h} \\
\mathbf{R}_{2}(t)=\mathbf{z}_{\xi}^{\mathrm{v}}[t-(t-h)][t-(t-2 h)][t-(t-3 h)]=6 \mathbf{z}_{\xi}^{\mathrm{V}} h^{3}=\mathrm{O}\left(h^{3}\right), \\
\xi \in(t-3 h, t)
\end{gathered}
$$

According to the polynomial and remainder expressions, the quadratic Lagrange extrapolation method is the most accurate, whereas the point approximation method is the most efficient.

The way the extrapolation strategy for relative rod accelerations is implemented depends on the specific time integration scheme under consideration. By defining the state vector as $\mathbf{y}^{\mathrm{T}}=\left\{\mathbf{z}^{\mathrm{T}}, \dot{\mathbf{z}}^{\mathrm{iT}}\right\}$ according to Maggi's approach [46], we can 
rewrite the final ODE system (3.16) in a generic way:

$$
\dot{\mathbf{y}} \equiv\left\{\begin{array}{c}
\dot{\mathbf{z}} \\
\ddot{\mathbf{z}}^{\mathrm{i}}
\end{array}\right\}=\mathbf{K}\left(t, \hat{\mathbf{z}},\left\{\begin{array}{c}
\mathbf{z} \\
\dot{\mathbf{z}}^{\mathrm{i}}
\end{array}\right\}\right) \equiv \mathbf{K}(t, \hat{\mathbf{z}}, \mathbf{y})
$$

which can be solved using standard integrators. Let us consider, as an example, a fixed-step, explicit, 4th-order Runge-Kutta scheme, which we will use in what follows:

$$
\begin{aligned}
\mathbf{y}_{i} & =\mathbf{y}_{i-1}+\frac{h}{6}\left(\mathbf{k}_{i}^{1}+2 \mathbf{k}_{i}^{2}+2 \mathbf{k}_{i}^{3}+\mathbf{k}_{i}^{4}\right) \\
\mathbf{k}_{i}^{1} & \equiv \mathbf{K}\left(t_{i-1}, \mathbf{y}_{i-1}\right) \\
\mathbf{k}_{i}^{2} & \equiv \mathbf{K}\left(t_{i-1}+\frac{h}{2}, \mathbf{y}_{i-1}+\frac{h}{2} \mathbf{k}_{i}^{1}\right) \\
\mathbf{k}_{i}^{3} & \equiv \mathbf{K}\left(t_{i-1}+\frac{h}{2}, \mathbf{y}_{i-1}+\frac{h}{2} \mathbf{k}_{i}^{2}\right) \\
\mathbf{k}_{i}^{4} & \equiv \mathbf{K}\left(t_{i-1}+h, \mathbf{y}_{i-1}+h \mathbf{k}_{i}^{3}\right)
\end{aligned}
$$

The geometric description of this scheme is shown in Figure 3.5. At timestep $i$ each of the state vector derivative evaluations $\left(\mathbf{k}_{i}^{1}, \mathbf{k}_{i}^{2}, \mathbf{k}_{i}^{3}\right.$ and $\left.\mathbf{k}_{i}^{4}\right)$ requires the extrapolation of the relative rod accelerations based on previous time points. For obvious reasons, the relative rod accelerations that are closest in time should be used. This means that in order to calculate $\mathbf{k}_{i}^{1}$ we can extrapolate based on accelerations up to $\mathbf{k}_{i-1}^{1}$, whereas in order to calculate $\mathbf{k}_{i}^{2}, \mathbf{k}_{i}^{3}$ and $\mathbf{k}_{i}^{4}$ we can extrapolate based on accelerations up to $\mathbf{k}_{i}^{1}$. Note that only true accelerations $\left(\mathbf{k}^{1}\right)$ can be used in the extrapolation, since intermediate accelerations $\mathbf{k}^{2}, \mathbf{k}^{3}$ and $\mathbf{k}^{4}$ are based on non-definitive integration points.

\subsection{Partitioning of rod mass matrix}

We have presented how the rod-related SDIF terms can be approximated for faster dynamic simulations. In systems that are heavily dependent on rods such as the sedan car previously analyzed, the loss of precision associated with rod-related 


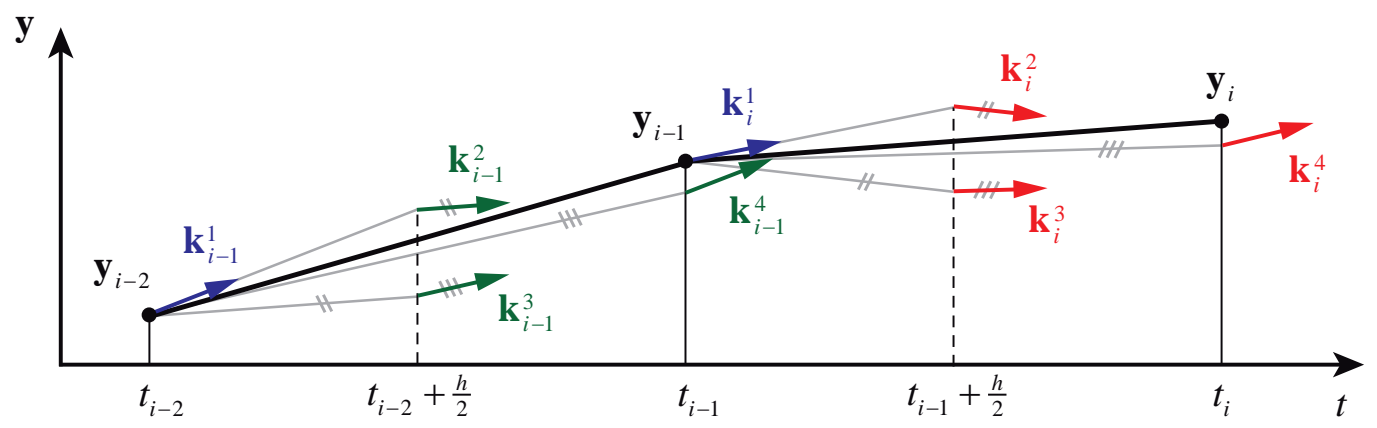

Figure 3.5: Geometric description of 4th-order Runge-Kutta integrator

SDIF approximation can lead to numerical inaccuracies that are higher than desired. In this section, we partition the rod mass matrix in order to reduce rod-related SDIF errors. This way, the solution accuracy can be improved while preserving computational efficiency.

The last term in the right-hand side of Equation (3.12), that is, the vector of accumulated approximate forces, contains the rod mass matrix. This matrix, in turn, can be regarded as the addition of diagonal and coupling terms:

$$
\mathbf{R}_{\mathrm{d}}^{\mathrm{T}} \mathbf{T}^{\mathrm{T}} \overline{\mathbf{M}}_{\text {rods }} \hat{\mathbf{Z}}=\mathbf{R}_{\mathrm{d}}^{\mathrm{T}} \mathbf{T}^{\mathrm{T}} \overline{\mathbf{M}}_{\text {rods }}^{\mathrm{d}} \hat{\mathbf{Z}}+\mathbf{R}_{\mathrm{d}}^{\mathrm{T}} \underbrace{\mathbf{T}^{\mathrm{T}} \overline{\mathbf{M}}_{\text {rods }}^{\mathrm{c}} \hat{\mathbf{Z}}_{\text {rods }}}_{\breve{\mathbf{F}}_{\text {rods }}^{\text {Tे }}}
$$

where $\overline{\mathbf{M}}_{\text {rods }}^{\mathrm{d}}$ and $\overline{\mathbf{M}}_{\text {rods }}^{\mathrm{c}}$ are, respectively, the diagonal and coupling terms of the rod mass matrix, which can be easily calculated. Vector $\breve{\mathbf{F}}_{\text {rods }}^{\Sigma}$ contains the coupling part of the approximate rod-related SDIFs.

Rearranging and taking into account Equation (1.21) we can derive the following set of dynamic equations:

$$
\begin{gathered}
\mathbf{R}_{\mathrm{z}}^{\mathrm{T}} \mathbf{R}_{\mathrm{d}}^{\mathrm{T}} \mathbf{M}^{\Sigma} \mathbf{R}_{\mathrm{d}} \mathbf{R}_{\mathrm{z}} \ddot{\mathbf{z}}^{\mathrm{i}}+\mathbf{R}_{\mathrm{z}}^{\mathrm{T}} \mathbf{R}_{\mathrm{d}}^{\mathrm{T}} \mathbf{M}_{\text {rods }}^{\mathrm{d} \Sigma}\left(\mathbf{R}_{\mathrm{d}} \mathbf{R}_{\mathrm{z}} \ddot{\mathbf{z}}^{\mathrm{i}}+\mathbf{R}_{\mathrm{d}} \dot{\mathbf{R}}_{\mathrm{z}} \dot{\mathbf{z}}^{\mathrm{i}}+\dot{\mathbf{R}}_{\mathrm{d}} \mathbf{R}_{\mathrm{z}} \dot{\mathbf{z}}^{\mathrm{i}}\right)= \\
\mathbf{R}_{\mathrm{z}}^{\mathrm{T}} \mathbf{R}_{\mathrm{d}}^{\mathrm{T}}\left(\hat{\mathbf{Q}}^{\Sigma}-\hat{\mathbf{P}}^{\Sigma}\right)-\mathbf{R}_{\mathrm{z}}^{\mathrm{T}} \mathbf{R}_{\mathrm{d}}^{\mathrm{T}} \breve{\mathbf{F}}_{\text {rods }}^{\Sigma}
\end{gathered}
$$

where $\mathbf{M}_{\text {rods }}^{\mathrm{d} \Sigma} \equiv \mathbf{T}^{\mathrm{T}} \overline{\mathbf{M}}_{\text {rods }}^{\mathrm{d}} \mathbf{T}$ is the diagonal component of the accumulated inertia 
matrix of the closed-loop system.

By introducing Equation (1.30) and rearranging, we can rewrite Equation (3.35) as:

$$
\begin{gathered}
\mathbf{R}_{\mathrm{z}}^{\mathrm{T}} \mathbf{R}_{\mathrm{d}}^{\mathrm{T}} \underbrace{\left(\mathbf{M}^{\Sigma}+\mathbf{M}_{\text {rods }}^{\mathrm{d} \Sigma}\right)}_{\hat{\mathbf{M}}_{\mathrm{d}}^{\Sigma}} \mathbf{R}_{\mathrm{d}} \mathbf{R}_{\mathrm{z}} \ddot{\mathbf{z}}^{\mathrm{i}}= \\
\mathbf{R}_{\mathrm{z}}^{\mathrm{T}} \mathbf{R}_{\mathrm{d}}^{\mathrm{T}}[\hat{\mathbf{Q}}^{\Sigma}-\mathbf{T}^{\mathrm{T}} \underbrace{\left(\overline{\mathbf{M}}+\overline{\mathbf{M}}_{\text {rods }}^{\mathrm{d}}\right)}_{\overline{\mathbf{M}}^{\mathrm{d}}} \frac{d\left(\mathbf{T} \mathbf{R}_{\mathrm{d}} \mathbf{R}_{\mathrm{z}}\right)}{d t} \dot{\mathbf{z}}^{\mathrm{i}}-\underbrace{\left(\mathbf{P}_{\text {rods }}^{\Sigma}+\breve{\mathbf{F}}_{\text {rods }}^{\Sigma}\right)}_{\breve{\mathbf{P}}_{\text {rods }}^{\Sigma}}]
\end{gathered}
$$

where $\hat{\mathbf{M}}_{\mathrm{d}}^{\Sigma}$ and $\overline{\mathbf{M}}^{\mathrm{d}}$ are the accumulated inertia matrix and the inertia matrix of the whole system, including the diagonal component of the rods' contribution.

Therefore, the approximate accumulated rod-related SDIFs (only containing coupling terms) can be described as:

$$
\breve{\mathbf{F}}_{\text {rods }}^{\Sigma}=\mathbf{T}^{\mathrm{T}} \overline{\mathbf{M}}_{\text {rods }}^{\mathrm{c}} \hat{\mathbf{Z}}_{\text {rods }}
$$

which, compared to the original accumulated rod-related SDIFs in Equation (3.19), is much smaller, thereby improving accuracy. Meanwhile, the system inertia matrix $\overline{\mathbf{M}}^{\mathrm{d}}$ in Equation (3.36) is still a diagonal matrix, which preserves the convenient structure of $\mathbf{R}_{\mathrm{d}}^{\mathrm{T}} \mathbf{M}^{\Sigma} \mathbf{R}_{\mathrm{d}}$ when we replace it by $\mathbf{R}_{\mathrm{d}}^{\mathrm{T}} \hat{\mathbf{M}}_{\mathrm{d}}^{\Sigma} \mathbf{R}_{\mathrm{d}}$.

In the vehicle example, the system inertia matrix including the rod mass matrix partition can be written as:

$$
\begin{aligned}
\overline{\mathbf{M}}^{\mathrm{d}}=\operatorname{diag}\left(\overline{\mathbf{M}}_{1}, \underline{\overline{\mathbf{M}}_{2}^{\prime}}, \overline{\mathbf{M}}_{3}, \ldots, \overline{\mathbf{M}}_{8}, \overline{\mathbf{M}}_{9}^{\prime}, \overline{\mathbf{M}}_{10}, \ldots, \overline{\mathbf{M}}_{14}, \overline{\mathbf{M}}_{15}^{\prime}, \overline{\mathbf{M}}_{16},\right. \\
\left.\overline{\mathbf{M}}_{17}, \underline{\overline{\mathbf{M}}_{18}^{\prime}}, \overline{\mathbf{M}}_{19}, \ldots, \overline{\mathbf{M}}_{23}, \overline{\mathbf{M}}_{24}^{\prime}, \overline{\mathbf{M}}_{25}, \ldots, \overline{\mathbf{M}}_{27}, \underline{\overline{\mathbf{M}}_{28}^{\prime}}, \overline{\mathbf{M}}_{29}, \ldots, \overline{\mathbf{M}}_{33}\right)
\end{aligned}
$$

where $\overline{\mathbf{M}}_{i}^{\prime} \equiv \overline{\mathbf{M}}_{i}+\overline{\mathbf{M}}_{\text {rod, } i}^{\mathrm{d}}$. These terms have been underlined for the sake of clarity. They can be computed by assembling the diagonal part of the rods' contribution presented in Table 3.6 onto the system inertia matrix, $\overline{\mathbf{M}}$. 
In short, the partitioning of the rod mass matrix brings about smaller rod-related SDIFs and preserves the structure of the mass matrix, which leads to more accurate results at a low computational cost.

\subsection{Results}

In order to verify the effectiveness and efficiency of the approximated rod-related SDIFs with respect to the different extrapolation methods, the 15-DOF sedan vehicle is simulated on a MATLAB/C/C++ environment, as shown in Figure 3.6, its system topology is described in Figure 3.1. Tire forces are modeled using Pacejka's Magic Formula. A comparative study has been carried out to assess solution accuracy and computational efficiency. All simulations consist of a slalom test along a flat road and are simulated using a 4th-order Runge-Kutta integrator and an initial velocity of $30 \mathrm{~m} / \mathrm{s}$.

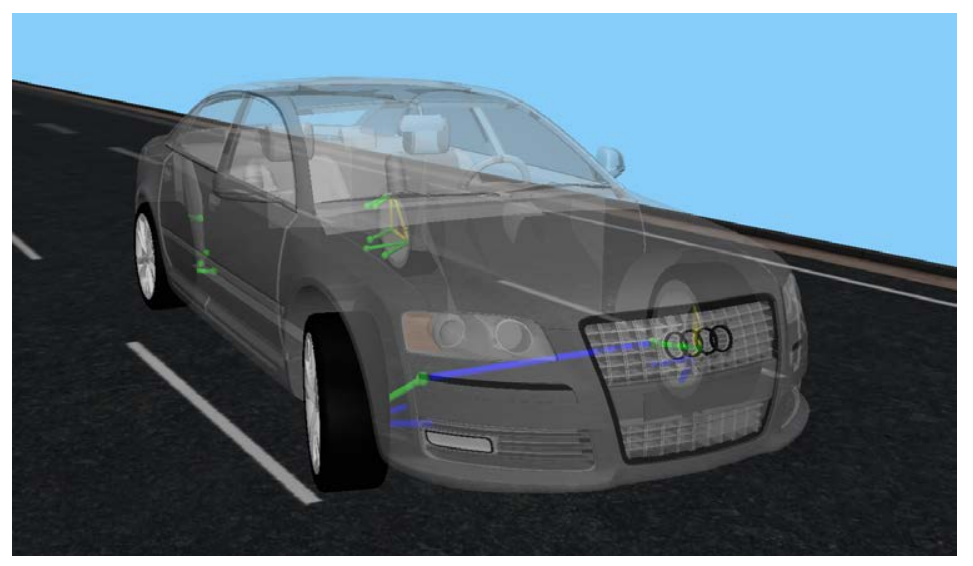

Figure 3.6: Multibody model of sedan vehicle 


\subsubsection{Accuracy}

A priori, we know that short time-steps, higher-order extrapolation methods and small rod masses will increase the solution accuracy when the rod-related SDIF approximation is used. The dynamic maneuver has been simulated for one second using the point approximation and linear and quadratic Lagrange extrapolation methods presented earlier. Different time-steps have been employed as well. To assess the accuracy of this method, system-wide relative accelerations are compared to those obtained from the original formulation in Equation (3.11). The maximum differences (errors) in relative accelerations are shown in Table 3.8. The errors increase with longer time-steps, and the largest error usually occurs at the end of the simulation because the differences build up over time. Also, the quadratic Lagrange extrapolation method provides the most accurate solutions.

\begin{tabular}{|c|c|c|c|c|c|c|}
\hline \multirow{3}{*}{$h(\mathrm{~ms})$} & \multicolumn{6}{|c|}{ Error } \\
\hline & \multicolumn{2}{|c|}{ Point approximation } & \multicolumn{2}{|c|}{ Linear Lagrange } & \multicolumn{2}{|c|}{ Quadratic Lagrange } \\
\hline & $\max (|\Delta \ddot{\mathbf{z}}|)$ & $\operatorname{norm}(\Delta \ddot{\mathbf{z}})$ & $\max (|\Delta \ddot{\mathbf{z}}|)$ & $\operatorname{norm}(\Delta \ddot{\mathbf{z}})$ & $\max (|\Delta \ddot{\mathbf{z}}|)$ & $\operatorname{norm}(\Delta \ddot{\mathbf{z}})$ \\
\hline 0.1 & $1.63 \times 10^{-4}$ & $3.50 \times 10^{-4}$ & $5.67 \times 10^{-7}$ & $1.28 \times 10^{-6}$ & $1.68 \times 10^{-8}$ & $3.07 \times 10^{-8}$ \\
\hline 0.2 & $3.24 \times 10^{-4}$ & $7.00 \times 10^{-3}$ & $2.33 \times 10^{-6}$ & $5.07 \times 10^{-6}$ & $1.11 \times 10^{-7}$ & $1.89 \times 10^{-7}$ \\
\hline 0.5 & $8.02 \times 10^{-4}$ & $1.70 \times 10^{-3}$ & $1.53 \times 10^{-5}$ & $3.18 \times 10^{-5}$ & $9.85 \times 10^{-7}$ & $2.05 \times 10^{-6}$ \\
\hline 1 & $1.60 \times 10^{-3}$ & $3.40 \times 10^{-3}$ & $6.56 \times 10^{-5}$ & $1.31 \times 10^{-4}$ & $5.29 \times 10^{-6}$ & $1.30 \times 10^{-5}$ \\
\hline
\end{tabular}

Table 3.8: Error incurred by rod-related SDIF approximation with different time-steps

The rod mass also affects the solution accuracy through the inertia matrix, as seen in Equations (3.19) and (3.37). Thus, we also investigate the accuracy with different rod mass values in order to assess the effectiveness of rod-related SDIFs approximation method. Table 3.9 shows the error when considering null, half, double and triple rod masses, respectively. Theoretically, the differences between the approximation method and the original formulation should be proportional to the rods' mass and null for the null rod mass case. The results in Table 3.9 corroborate this and show that the errors are very small even with artificially heavy 
rods. Linear and quadratic extrapolation methods are much more accurate than the point approximation case. The error, as expected, increases with longer time-steps and builds up over time.

\begin{tabular}{cccccccc}
\hline \multirow{2}{*}{ Mass } & $h(\mathrm{~ms})$ & \multicolumn{5}{c}{ Error } \\
\cline { 3 - 8 } & & \multicolumn{2}{c}{ Point approximation } & \multicolumn{2}{c}{ Linear Lagrange } & \multicolumn{2}{c}{ Quadratic Lagrange } \\
\cline { 3 - 8 } & & $\max (|\Delta \ddot{\mathbf{z}}|)$ & $\operatorname{norm}(\Delta \ddot{\mathbf{z}})$ & $\max (|\Delta \ddot{\mathbf{z}}|)$ & $\operatorname{norm}(\Delta \ddot{\mathbf{z}})$ & $\max (|\Delta \ddot{\mathbf{z}}|)$ & $\operatorname{norm}(\Delta \ddot{\mathbf{z}})$ \\
\hline \multirow{2}{*}{0} & 0.5 & 0 & 0 & 0 & 0 & 0 & 0 \\
& 1 & 0 & 0 & 0 & 0 & 0 & 0 \\
\hline \multirow{2}{*}{$0.5 \times$} & 0.5 & $4.3 \times 10^{-4}$ & $9.2 \times 10^{-4}$ & $7.5 \times 10^{-6}$ & $1.6 \times 10^{-5}$ & $4.8 \times 10^{-7}$ & $9.8 \times 10^{-7}$ \\
& 1 & $8.5 \times 10^{-4}$ & $1.8 \times 10^{-3}$ & $3.1 \times 10^{-5}$ & $6.5 \times 10^{-5}$ & $2.7 \times 10^{-6}$ & $6.6 \times 10^{-6}$ \\
\hline \multirow{2}{*}{$2 \times$} & 0.5 & $1.3 \times 10^{-3}$ & $3.1 \times 10^{-3}$ & $3.4 \times 10^{-5}$ & $6.4 \times 10^{-5}$ & $1.9 \times 10^{-6}$ & $4.0 \times 10^{-6}$ \\
& 1 & $2.6 \times 10^{-3}$ & $6.1 \times 10^{-3}$ & $1.4 \times 10^{-4}$ & $2.6 \times 10^{-4}$ & $8.8 \times 10^{-6}$ & $2.3 \times 10^{-5}$ \\
\hline \multirow{2}{*}{$3 \times$} & 0.5 & $3.0 \times 10^{-3}$ & $5.4 \times 10^{-3}$ & $1.1 \times 10^{-4}$ & $2.2 \times 10^{-4}$ & $1.7 \times 10^{-5}$ & $2.5 \times 10^{-5}$ \\
& 1 & $3.6 \times 10^{-3}$ & $8.4 \times 10^{-3}$ & $2.3 \times 10^{-4}$ & $3.8 \times 10^{-4}$ & $1.5 \times 10^{-5}$ & $3.5 \times 10^{-5}$ \\
\hline
\end{tabular}

Table 3.9: Error incurred by rod-related SDIF approximation with different rod masses

\subsubsection{Efficiency}

The dynamic simulation has been timed for each of the approximation methods. All simulations have been run on an Intel $\AA$ Xeon $\AA$ machine with a $3.0 \mathrm{GHz}$ CPU and $4 \mathrm{~GB}$ of RAM. Table 3.10 shows the elapsed times for the different rod-related SDIF approximation techniques, as well as the original semi-recursive formulation.

The computational savings take place in the state vector derivative function, which is the one directly affected by rod-related SDIF approximations. When using a 0.5 -ms integration time-step, the point, linear and quadratic Lagrange approximation methods improve the computational efficiency by $10.14 \%, 9.78 \%$ and $9.17 \%$, respectively. In the 1-ms time-step case, the computational efficiency is improved by $10.44 \%, 10.03 \%$ and $9.83 \%$. The quadratic Lagrange extrapolation method yields the most accurate solutions. 


\begin{tabular}{llccc}
\hline$h(\mathrm{~ms})$ & Formulation & Loop count & $\begin{array}{c}\text { Elapsed } \\
\text { time (s) }\end{array}$ & Improvement \\
\hline \multirow{2}{*}{$0.5 \quad$ Original formulation } & & 11.577 & - \\
& Point approximation & 80000 & 10.403 & $\mathbf{1 0 . 1 4 \%}$ \\
& Linear Lagrange extrapolation & & 10.444 & $\mathbf{9 . 7 8 \%}$ \\
& Quadratic Lagrange extrapolation & & 10.515 & $\mathbf{9 . 1 7 \%}$ \\
\hline \multirow{2}{*}{$1 \quad$ Original formulation } & & 11.586 & - \\
& Point approximation & \multirow{2}{*}{80000} & 10.376 & $\mathbf{1 0 . 4 4 \%}$ \\
& Linear Lagrange extrapolation & & 10.424 & $\mathbf{1 0 . 0 3 \%}$ \\
& Quadratic Lagrange extrapolation & & 10.447 & $\mathbf{9 . 8 3 \%}$ \\
\hline
\end{tabular}

Table 3.10: Computational efficiency of rod-related SDIF approximation during 10-s and 20-s simulations

\subsection{Discussion}

We have presented an enhancement to the double-step, semi-recursive formulation developed in [43]. First, the topology of a 15-DOF vehicle model has been fully described and modeled through the aforementioned formulation, including the cut-joint and rod-removal techniques. Second, an original method for approximating rod-related, second-derivative-based inertia forces (SDIF) has been presented, which has improved computational efficiency. Three extrapolation methods have been introduced as part of the rod-related SDIF approximation: point approximation, and linear and quadratic Lagrange extrapolation. Third, the mass matrix of the rods has been partitioned into diagonal and off-diagonal elements to ameliorate the accuracy of the rod-related SDIF approximation. The efficacy and efficiency of the presented approach has been assessed through a series of dynamic simulations where the extrapolation method, the rod mass and the time-step have been varied. An efficiency gain of about $10 \%$ has been observed, along with a satisfactory accuracy. Overall, a very simple yet effective approximation technique has been presented in the context of efficient formulations for rod modeling in vehicle sys- 
tems. Systems with more rods or systems depending more heavily on rod inertial forces can greatly benefit from this technique. 


\section{Chapter 4}

\section{Iterative refinement of accelerations}

In this Chapter, an iterative refinement technique is introduced into a 4th-order Runge-Kutta integrator within the double-step semi-recursive formulation. It will improve efficiency without losing accuracy. The basic principle of iterative refinement is to solve the dynamic equations iteratively while maintaining the acyclic solution constraints met. The iterative process is applied to compute corrections of the solution in an economic way, terminating as soon as a given precision at the solution is reached. It starts from an initial guess close to the exact solution, which ensures few iterations and thus leads to efficient iterative refinement.

\subsection{Introduction}

The iterative refinement is a well-established technique for improving the accuracy of a system of linear equations $\mathbf{A x}=\mathbf{b}$. It was proposed by Wilkinson [25, 114] and then widely used and extended by several authors [40, 60, 78, 104, 106]. Given some basic solution method such as Gaussian elimination with partial pivoting, the basic iterative refinement algorithm can be described as $[33,58]$ : 
- Input: A matrix $\mathbf{A} \in \mathbb{R}^{n \times n}$ and a vector $\mathbf{b} \in \mathbb{R}^{n}$

- Solve $\mathbf{A} \mathbf{x}^{k}=\mathbf{b}$ through Gaussian elimination with partial pivoting (initial guess: $k=0$ )

- Repeat

1. Compute residual $\mathbf{r}^{k}=\mathbf{A} \mathbf{x}^{k}-\mathbf{b}$

2. Solve $\mathbf{A} d \mathbf{x}^{k+1}=\mathbf{r}^{k}$ through Gaussian elimination with partial pivoting

3. Update $\mathbf{x}^{k+1}=\mathbf{x}^{k}-d \mathbf{x}^{k+1}$

4. $k=k+1$

- Until $\mathbf{x}^{k}$ is accurate enough

- Output: A solution vector $\mathbf{x}^{k}$ approximating $\mathbf{x}$ in $\mathbf{A x}=\mathbf{b}$, and an error bound $\varepsilon=\left\|\mathbf{x}^{k}-\mathbf{x}^{k-1}\right\|_{\infty} /\left\|\mathbf{x}^{k-1}\right\|_{\infty}$

If there are no rounding errors in the computation of vectors $\mathbf{r}$ and $d \mathbf{x}$, then vector $\mathbf{x}$ will be the exact solution of the linear system. The idea behind the iterative refinement is that a number of improvements of the accuracy will be obtained if vectors $\mathbf{r}$ and $d \mathbf{x}$ are computed accurately enough. The economics of the iterative refinement is favorable for solvers based on the factorization of leading matrix $\mathbf{A}$. This matrix factorization used to compute vector $d \mathbf{x}^{1}$ in the first iteration can be reused in the rest.

Originally, Gaussian elimination was applied for the matrix factorization and vector $\mathbf{r}$ was computed in double precision before being rounded to working precision. If double precision was used in the computation of vector $\mathbf{r}$, and the leading matrix A was not too ill-conditioned, then the iterative refinement produced a solution correct to working precision, and the rate of convergence depended on the condition number of leading matrix $\mathbf{A}[9,60]$.

Floating-point precision represented the working precision in the original iterative refinement algorithm due to the limitation of memory storage in the sixties 
[25, 114]. However, in recent times, double precision is the standard working precision. A variety of efficient alternative algorithms such as the Chebyshev algorithm have been developed based on the basic iterative refinement algorithm. The Chebyshev iteration accelerates the process without loss of numerical stability $[10,48,51,52]$. In this Chapter, a modified iterative refinement algorithm is introduced to speed up an explicit 4th-order Runge-Kutta integrator. The main advantage of the presented algorithm is the reuse of the generalized mass matrix factorization between neighboring Runge-Kutta steps.

\subsection{Algorithm}

By grouping some of the terms, the equations of motion of a closed-loop system in Equation (1.31) can be rewritten in compact form:

$$
\begin{aligned}
\breve{\mathbf{M}}(\mathbf{z}) \ddot{\mathbf{z}}^{\mathrm{i}}(t) & =\breve{\mathbf{F}}(t, \mathbf{z}, \dot{\mathbf{z}}) \\
\breve{\mathbf{M}}(\mathbf{z}) & \equiv \mathbf{R}_{\mathrm{z}}^{\mathrm{T}} \mathbf{R}_{\mathrm{d}}^{\mathrm{T}} \mathbf{M}^{\Sigma} \mathbf{R}_{\mathrm{d}} \mathbf{R}_{\mathrm{z}} \\
\breve{\mathbf{F}}(t, \mathbf{z}, \dot{\mathbf{z}}) & \equiv \mathbf{R}_{\mathrm{z}}^{\mathrm{T}} \mathbf{R}_{\mathrm{d}}^{\mathrm{T}} \mathbf{Q}^{\Sigma}-\mathbf{R}_{\mathrm{z}}^{\mathrm{T}} \mathbf{R}_{\mathrm{d}}^{\mathrm{T}} \mathbf{T}^{\mathrm{T}} \overline{\mathbf{M}} \mathbf{D}
\end{aligned}
$$

Let $\mathbf{y}$ be a vector containing the current system state variables. Taking into account the initial values of the state variables, the general form of the equations of motion (Equation (4.1)) can be expressed as a first-order system:

$$
\left\{\begin{array}{l}
\dot{\mathbf{y}}=\mathbf{f}(t, \mathbf{y}) \\
\left.\mathbf{y}\right|_{t=0}=\mathbf{y}_{0}
\end{array}\right.
$$

By introducing Equation (4.1), Equation (4.4) takes the following expression:

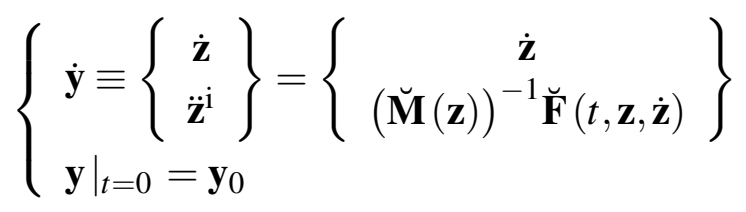


The equations of motion in Equation (4.5) can be integrated over time using time integration algorithms. An explicit 4th-order Runge-Kutta integrator with a constant time-step was introduced in Chapter 2. Experience has showed that this explicit integrator is a good trade-off between computational efficiency and ease of implementation $[43,45,46]$.

Let us consider the geometric description of the 4th-order Runge-Kutta integrator (recall Figure 3.5). The generalized mass matrix $\breve{\mathbf{M}}$ on the left hand side of the equations of motion varies very slowly between the second and third, as well as between the fourth and next first Runge-Kutta steps. Let us consider the sedan vehicle that was modeled in Section 2.4 and the semi-trailer truck that will be modeled in Section 4.3 as examples. The Euclidean norm of the differences in the generalized mass matrix between neighboring Runge-Kutta steps with different time-steps are gathered in Table 4.1.

Thus the generalized mass matrix in the second and fourth Runge-Kutta steps is factorized, and then the factorization is reused in the rest of the steps. Iterative refinement is applied on the first and third Runge-Kutta steps after reusing the generalized mass matrix factorization. First, the initial guess of the solution (independent relative accelerations) is produced. Second, the iterative refinement process is carried out on the basis of the initial guess and the generalized mass matrix factorization. This consists of three substeps: residual calculation, solution increment calculation and solution update. Third and last, an appropriate termination criteria is set up to control the refinement process.

\subsubsection{Initial value determination}

Let us recall Equation (4.1), an explicit 4th-order Runge-Kutta integrator is used to solve the equations, with four steps per integration step. This was explained in Chapter 3. In the second and fourth Runge-Kutta steps, the generalized mass 


\begin{tabular}{cccccc}
\hline \multirow{2}{*}{ Model } & \multirow{2}{*}{$h(\mathrm{~ms})$} & \multicolumn{4}{c}{$\operatorname{Norm}(\Delta \breve{\mathbf{M}})$ between R-K steps } \\
\cline { 3 - 6 } & & 1 st-2nd & 2nd-3rd & 3rd-4th & 4th-5th (next 1st) \\
\hline \multirow{4}{*}{ Sedan } & 0.1 & $2.24 \times 10^{-4}$ & $1.12 \times 10^{-4}$ & $3.35 \times 10^{-4}$ & $2.00 \times 10^{-7}$ \\
& 0.2 & $8.92 \times 10^{-4}$ & $4.44 \times 10^{-4}$ & $1.33 \times 10^{-3}$ & $1.60 \times 10^{-6}$ \\
& 0.5 & $5.53 \times 10^{-3}$ & $2.72 \times 10^{-3}$ & $8.17 \times 10^{-3}$ & $2.49 \times 10^{-5}$ \\
& 1 & $2.17 \times 10^{-2}$ & $1.05 \times 10^{-2}$ & $3.16 \times 10^{-2}$ & $1.97 \times 10^{-4}$ \\
\hline \multirow{4}{*}{ Truck } & 0.1 & $3.12 \times 10^{-3}$ & $1.42 \times 10^{-3}$ & $4.30 \times 10^{-3}$ & $8.92 \times 10^{-5}$ \\
& 0.2 & $1.15 \times 10^{-2}$ & $4.80 \times 10^{-3}$ & $1.46 \times 10^{-2}$ & $6.51 \times 10^{-4}$ \\
& 0.5 & $5.64 \times 10^{-2}$ & $1.79 \times 10^{-2}$ & $5.85 \times 10^{-2}$ & $7.53 \times 10^{-3}$ \\
& 1 & $1.40 \times 10^{-1}$ & $4.65 \times 10^{-2}$ & $1.02 \times 10^{-1}$ & $5.32 \times 10^{-2}$ \\
\hline
\end{tabular}

Table 4.1: The changes of the generalized mass matrix between neighboring Runge-Kutta steps with different time-steps

matrix is factorized through Cholesky factorization:

$$
\begin{aligned}
& \breve{\mathbf{M}}\left(t_{i}+\frac{h}{2}\right) \equiv \mathbf{L}_{2} \mathbf{L}_{2}^{\mathrm{T}} \\
& \breve{\mathbf{M}}\left(t_{i}+h\right) \equiv \mathbf{L}_{4} \mathbf{L}_{4}^{\mathrm{T}}
\end{aligned}
$$

where $\mathbf{L}_{2}$ and $\mathbf{L}_{4}$ are lower triangular matrices corresponding to the second and fourth Runge-Kutta steps, respectively. Thus the corresponding independent relative accelerations, $\ddot{\mathbf{z}}_{2}^{\mathrm{i}}$ and $\ddot{\mathbf{z}}_{4}^{\mathrm{i}}$, are obtained:

$$
\begin{aligned}
\mathbf{L}_{2}^{\mathrm{T}} \ddot{\mathbf{z}}_{2}^{\mathrm{i}}=\breve{\mathbf{x}}_{2}, & \mathbf{L}_{2} \breve{\mathbf{x}}_{2}=\breve{\mathbf{F}}\left(t_{i}+\frac{h}{2}, \mathbf{z}_{2}, \dot{\mathbf{z}}_{2}\right) \\
\mathbf{L}_{4}^{\mathrm{T}} \ddot{\mathbf{z}}_{4}^{\mathrm{i}}=\breve{\mathbf{x}}_{4}, & \mathbf{L}_{4} \breve{\mathbf{x}}_{4}=\breve{\mathbf{F}}\left(t_{i}+h, \mathbf{z}_{4}, \dot{\mathbf{z}}_{4}\right)
\end{aligned}
$$

where $\mathbf{z}_{i}$ and $\dot{\mathbf{z}}_{i}$ represent the dependent relative positions and velocities of the $i$-th Runge-Kutta step.

In order to obtain the initial guess of the iterative refinement in the first and third Runge-Kutta steps, the matrix factorization $\left(\mathbf{L}_{2} \mathbf{L}_{2}^{\mathrm{T}}\right.$ and $\left.\mathbf{L}_{4} \mathbf{L}_{4}^{\mathrm{T}}\right)$ is reused. The 
corresponding initial guess $\left(\ddot{\mathbf{z}}_{3}^{\mathrm{i}}\right.$ and $\left.\ddot{\mathbf{z}}_{1}^{\mathrm{i}}\right)$ is computed:

$$
\begin{array}{ll}
\mathbf{L}_{2}^{\mathrm{T}} \ddot{\mathbf{z}}_{3}^{\mathrm{i}}=\breve{\mathbf{x}}_{3}, & \mathbf{L}_{2} \breve{\mathbf{x}}_{3}=\breve{\mathbf{F}}\left(t_{i}+\frac{h}{2}, \mathbf{z}_{3}, \dot{\mathbf{z}}_{3}\right) \\
\mathbf{L}_{4}^{\mathrm{T}} \ddot{\mathbf{z}}_{1}^{\mathrm{i}}=\breve{\mathbf{x}}_{1}, & \mathbf{L}_{4} \breve{\mathbf{x}}_{1}=\breve{\mathbf{F}}\left(t_{i}+h, \mathbf{z}_{1}, \dot{\mathbf{z}}_{1}\right)
\end{array}
$$

Considering small changes in the generalized mass matrix between neighboring Runge-Kutta steps (recall Table 4.1), the initial guess should be close to the exact solution. Tables 4.2 gathers the differences between the initial guess and the exact solution (produced by the exact procedure with a 0.1-ms time-step).

\subsubsection{Iterative refinement process}

Let the initial guess at time $t$ be written as $\left(\ddot{\mathbf{z}}^{\mathrm{i}}\right)^{0}$. Equation (4.1) at time $t$ can be solved based on the initial guess through an iterative refinement process. the 4th-order Runge-Kutta procedure with iterative refinement is described Figure 4.1, and Figure 4.2 shows the iterative refinement procedure. The process consists of residual calculation, solution increment calculation and solution update. The residual is calculated as:

$$
\begin{aligned}
& \mathbf{r}^{k}=\breve{\mathbf{M}}\left(\ddot{\mathbf{z}}^{\mathrm{i}}\right)^{k}-\breve{\mathbf{F}} \equiv \mathbf{R}_{\mathrm{z}}^{\mathrm{T}} \overline{\mathbf{M}}^{\Sigma} \mathbf{R}_{\mathrm{z}}\left(\ddot{\mathbf{z}}^{\mathrm{i}}\right)^{k}-\breve{\mathbf{F}} \\
& \overline{\mathbf{M}}^{\Sigma} \equiv \mathbf{R}_{\mathrm{d}}^{\mathrm{T}} \mathbf{M}^{\Sigma} \mathbf{R}_{\mathrm{d}}
\end{aligned}
$$

where $\mathbf{r}^{k}$ and $\left(\ddot{\mathbf{z}}^{\mathrm{i}}\right)^{k}$ represent the residual and the independent relative accelerations of the $(k+1)$-th iteration and $\overline{\mathbf{M}}^{\Sigma}$ is the composite mass matrix.

Note that the generalized mass matrix $\breve{\mathbf{M}}$, which involves expensive matrixmatrix operations, is not calculated. The first term on the right hand side of Equation (4.12), $\mathbf{R}_{\mathrm{z}}^{\mathrm{T}} \overline{\mathbf{M}}^{\Sigma} \mathbf{R}_{\mathrm{z}}\left(\ddot{\mathbf{z}}^{\mathrm{i}}\right)^{k}$, is computed from the right to left by using matrixvector operations, which is more efficient than matrix-matrix operations. This 


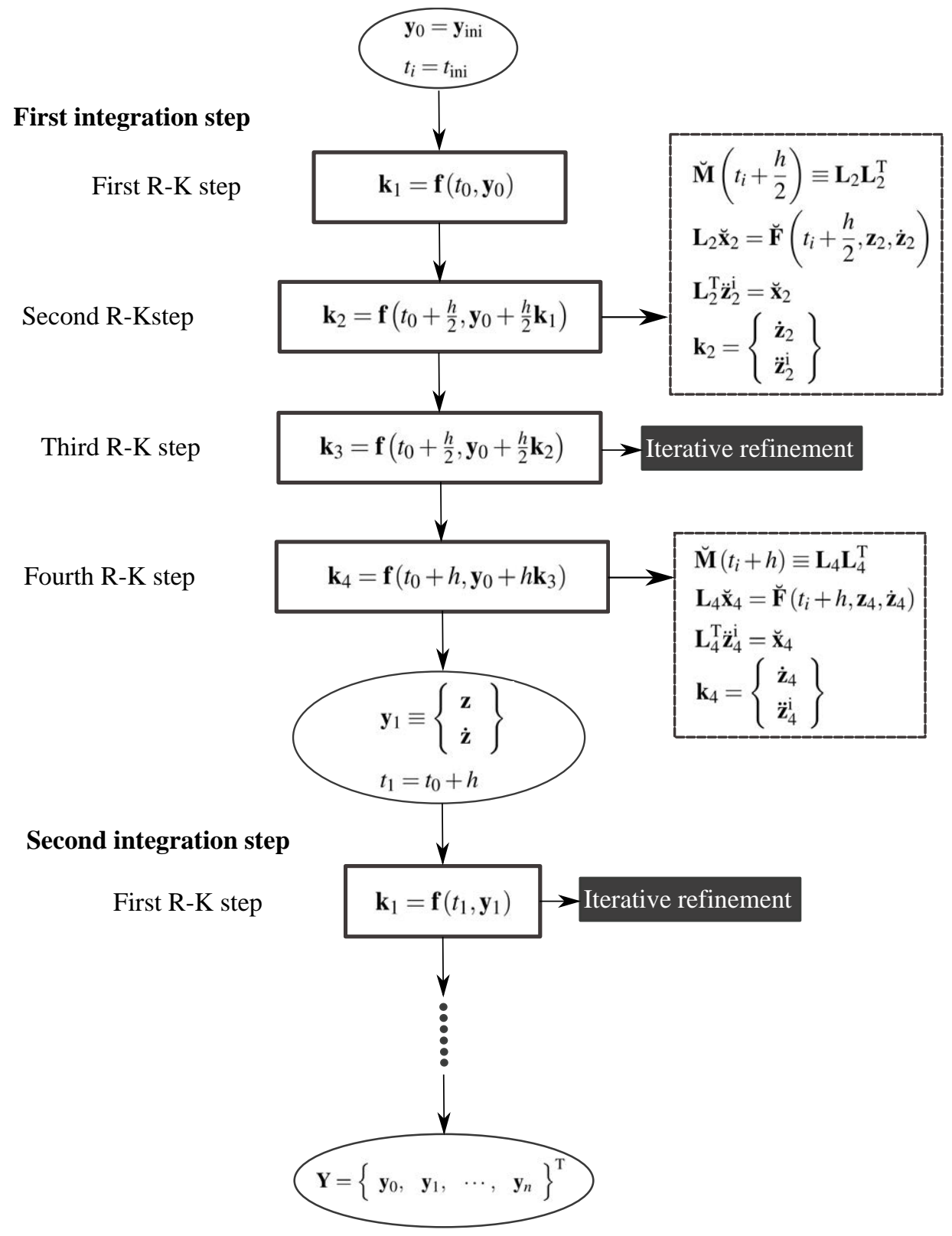

Figure 4.1: 4th-order Runge-Kutta procedure with iterative refinement technique 


\begin{tabular}{|c|c|c|c|c|c|}
\hline \multirow{3}{*}{ Model } & \multirow{3}{*}{$h(\mathrm{~ms})$} & \multicolumn{4}{|c|}{ Differences } \\
\hline & & \multicolumn{2}{|c|}{ 3th R-K step } & \multicolumn{2}{|c|}{ 1st R-K step } \\
\hline & & $\max \left(\left|\Delta \ddot{\mathbf{z}}^{\mathrm{i}}\right|\right)$ & $\frac{\operatorname{norm}\left(\Delta \ddot{\mathbf{z}}^{\mathrm{i}}\right)}{\operatorname{norm}\left(\ddot{\mathbf{z}}^{\mathrm{i}}\right)}$ & $\max \left(\left|\Delta \ddot{\mathbf{z}}^{\mathrm{i}}\right|\right)$ & $\frac{\operatorname{norm}\left(\Delta \ddot{\mathbf{z}}^{\mathrm{i}}\right)}{\operatorname{norm}\left(\ddot{\mathbf{z}}^{\mathrm{i}}\right)}$ \\
\hline \multirow{4}{*}{ Sedan } & 0.1 & $3.22 \times 10^{-4}$ & $1.07 \times 10^{-6}$ & $4.94 \times 10^{-7}$ & $1.71 \times 10^{-9}$ \\
\hline & 0.2 & $1.30 \times 10^{-3}$ & $4.29 \times 10^{-6}$ & $3.92 \times 10^{-6}$ & $1.36 \times 10^{-8}$ \\
\hline & 0.5 & $8.00 \times 10^{-3}$ & $2.67 \times 10^{-5}$ & $6.00 \times 10^{-5}$ & $2.10 \times 10^{-7}$ \\
\hline & 1 & $3.15 \times 10^{-2}$ & $1.06 \times 10^{-4}$ & $4.62 \times 10^{-4}$ & $1.64 \times 10^{-6}$ \\
\hline \multirow{4}{*}{ Truck } & 0.1 & $5.86 \times 10^{-7}$ & $2.69 \times 10^{-10}$ & $3.57 \times 10^{-8}$ & $1.44 \times 10^{-11}$ \\
\hline & 0.2 & $2.19 \times 10^{-6}$ & $1.06 \times 10^{-9}$ & $2.63 \times 10^{-7}$ & $1.24 \times 10^{-10}$ \\
\hline & 0.5 & $1.20 \times 10^{-5}$ & $6.31 \times 10^{-9}$ & $3.12 \times 10^{-6}$ & $2.40 \times 10^{-9}$ \\
\hline & 1 & $5.05 \times 10^{-5}$ & $2.40 \times 10^{-8}$ & $1.75 \times 10^{-5}$ & $2.32 \times 10^{-8}$ \\
\hline
\end{tabular}

Table 4.2: The differences between the initial guess and the exact solution

process is described as:

$$
\begin{aligned}
& \mathbf{R}_{\mathrm{z}}^{\mathrm{T}} \overline{\mathbf{M}}^{\Sigma} \mathbf{R}_{\mathrm{z}}\left(\ddot{\mathbf{z}}^{\mathrm{i}}\right)^{k}=\mathbf{R}_{\mathrm{z}}^{\mathrm{T}} \boldsymbol{\varepsilon} \\
& \boldsymbol{\varepsilon}=\overline{\mathbf{M}}^{\Sigma} \boldsymbol{\gamma} \\
& \boldsymbol{\gamma}=\mathbf{R}_{\mathrm{z}}\left(\ddot{\mathbf{z}}^{\mathrm{i}}\right)^{k}
\end{aligned}
$$

where $\boldsymbol{\varepsilon}$ and $\boldsymbol{\gamma}$ are auxiliary vectors introduced in above matrix-vector operations.

The solution increment of the $(k+1)$-th iteration, $d\left(\ddot{\mathbf{z}}^{\mathrm{i}}\right)^{k+1}$, is obtained:

$$
\begin{aligned}
& \mathbf{L}^{\mathrm{T}} d\left(\ddot{\mathbf{z}}^{\mathrm{i}}\right)^{k+1}=\breve{\mathbf{x}} \\
& \mathbf{L} \breve{\mathbf{x}}=\mathbf{r}^{k}
\end{aligned}
$$

where $\mathbf{L L}^{\mathrm{T}}$ is the generalized mass matrix factorization obtained from the previous Runge-Kutta step. The calculation of the generalized mass matrix and its factorization are avoided in the iterative refinement process.

Taking into account the solution increment, the solution of the $(k+1)$-th 


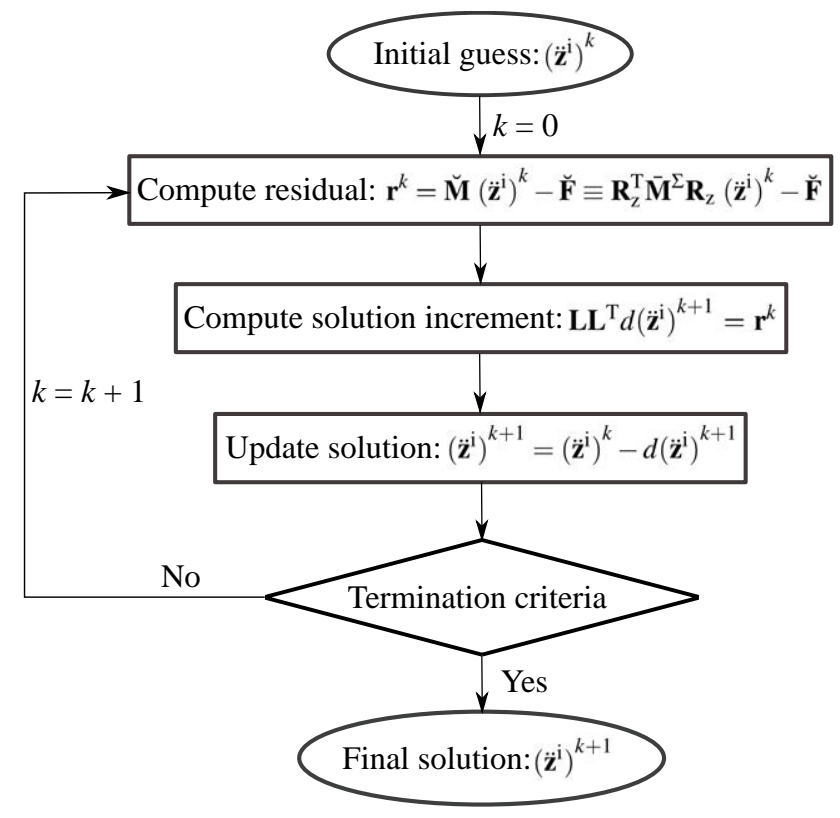

Figure 4.2: Iterative refinement procedure

iteration is updated:

$$
\left(\ddot{\mathbf{z}}^{\mathrm{i}}\right)^{k+1}=\left(\ddot{\mathbf{z}}^{\mathrm{i}}\right)^{k}-d\left(\ddot{\mathbf{z}}^{\mathrm{i}}\right)^{k+1}
$$

The iterative refinement process is repeated until the solution meets the termination criteria. According to the simulation results of the sedan vehicle and semi-trailer truck, very few iterations can lead to an accurate solution. Furthermore, fewer iterations are required for shorter time-steps.

Note that in the double-step semi-recursive formulation, the generalized mass matrix on the left hand side of the equations of motion is always positive definite. Hence, Cholesky factorization, instead of Gaussian elimination, has been implemented to factorize the generalized mass matrix [32]. 


\subsubsection{Termination criteria}

In order to control the iterative refinement process, the termination criteria or the specifications of the accuracy to which each iteration is performed is set up. Let the forward error of the $k$-th iteration be denoted by $\mathbf{e}_{k}=\left(\ddot{\mathbf{z}}^{\mathrm{i}}\right)^{k}-\left(\ddot{\mathbf{z}}^{\mathrm{i}}\right)^{k-1}$. A weighted error norm is introduced [57]:

$$
\begin{aligned}
& \varepsilon=\sqrt{\frac{1}{n} \sum_{k=1}^{n}\left(\frac{\mathbf{e}_{k}}{\mathbf{w}_{k}}\right)^{2}} \\
& \mathbf{w}_{k}=c_{1}\left|\left(\ddot{\mathbf{z}}^{\mathrm{i}}\right)^{k}\right|+c_{2}
\end{aligned}
$$

where $c_{1}$ and $c_{2}$ represent the relative and absolute tolerance, respectively. These parameters can be different for each type of variables, such as linear translations and rotations. The weighted error norm reaches convergence when $\varepsilon<1$.

By introducing the initial guess and the termination criteria, the iterative refinement process is complete:

- Input:

- Composite mass matrix $\overline{\mathbf{M}}^{\Sigma} \in \mathbb{R}^{n \times n}$

- The second velocity transformation matrix $\mathbf{R}_{\mathrm{Z}} \in \mathbb{R}^{n \times f}$

- Generalized force vector $\breve{\mathbf{F}} \in \mathbb{R}^{n}$

- Generalized mass matrix factorization $\mathbf{L L}^{\mathrm{T}}$ from the previous RungeKutta step $\left(\mathbf{R}_{\mathrm{Z}}^{\mathrm{T}} \overline{\mathbf{M}}^{\Sigma} \mathbf{R}_{\mathrm{z}} \cong \mathbf{L} \mathbf{L}^{\mathrm{T}}\right)$

- Solve $\mathbf{R}_{\mathrm{z}}^{\mathrm{T}} \overline{\mathbf{M}}^{\Sigma} \mathbf{R}_{\mathrm{z}}\left(\ddot{\mathbf{z}}^{\mathrm{i}}\right)^{k}=\breve{\mathbf{F}}$ (initial guess: $k=0$ )

- Repeat

1. Compute residual $\mathbf{r}^{k}=\mathbf{R}_{\mathrm{z}}^{\mathrm{T}} \overline{\mathbf{M}}^{\Sigma} \mathbf{R}_{\mathrm{z}}\left(\ddot{\mathbf{z}}^{\mathrm{i}}\right)^{k}-\breve{\mathbf{F}}$

2. Solve $\left(\mathbf{L} \mathbf{L}^{\mathrm{T}}\right) d\left(\ddot{\mathbf{z}}^{\mathrm{i}}\right)^{k+1}=\mathbf{r}^{k}$

3. Update $\left(\ddot{\mathbf{z}}^{\mathrm{i}}\right)^{k+1}=\left(\ddot{\mathbf{z}}^{\mathrm{i}}\right)^{k}-d\left(\ddot{\mathbf{z}}^{\mathrm{i}}\right)^{k+1}$ 
4. $k=k+1$

- Until $\left(\ddot{\mathbf{z}}^{\mathrm{i}}\right)^{k}$ satisfies $\varepsilon<1$

- Output:

- A solution $\left(\ddot{\mathbf{z}}^{\mathrm{i}}\right)^{k}$ approximating $\ddot{\mathbf{z}}^{\mathrm{i}}$ in $\mathbf{R}_{\mathrm{z}}^{\mathrm{T}} \overline{\mathbf{M}}^{\Sigma} \mathbf{R}_{\mathrm{z}} \ddot{\mathbf{z}}^{\mathrm{i}}=\breve{\mathbf{F}}$

- A weighted error norm $\varepsilon=\sqrt{\frac{1}{n} \sum_{k=1}^{n}\left(\frac{\mathbf{e}_{k}}{\mathbf{w}_{k}}\right)^{2}}$

\subsection{Results}

In order to investigate the accuracy and computational efficiency of the presented iterative refinement algorithm, a medium-size 16-DOF sedan vehicle model (see Chapter 2) is simulated here with different time-steps. Further, a large-size 40-DOF semi-trailer truck model is simulated to investigate how the size of the vehicle system affects the efficiency. A slalom test maneuver along a flat road is performed in all dynamic simulations.

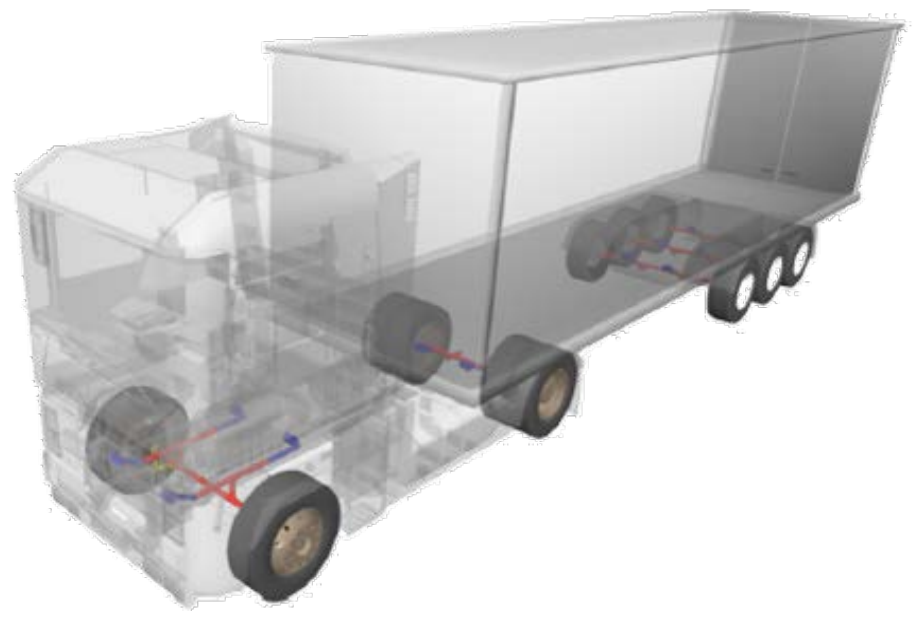

Figure 4.3: Truck multibody model (courtesy of A. F. Hidalgo and A. Callejo) 


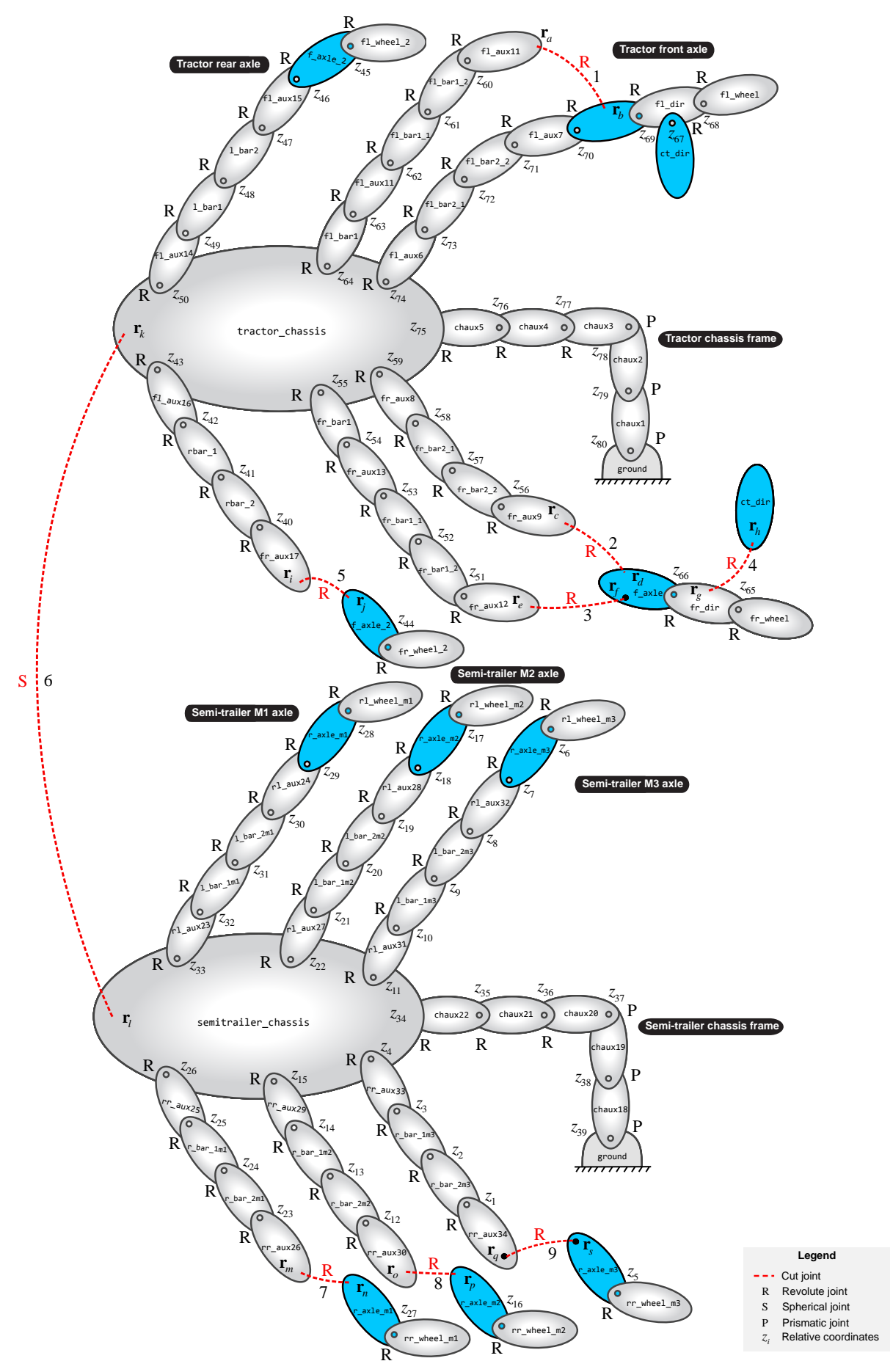

Figure 4.4: Truck system topology 
The truck multibody model is described in Figure 4.3, consisting of a complete truck system with tractor and semi-trailer [46, 57]. Figure 4.4 describes its treediagram topology, where several auxiliary massless bodies are introduced to model spherical, universal and 6-DOF joints by means of 1-DOF revolute and prismatic joints. In order to make full use of recursive kinematics, certain joints are cut to open this closed-loop system. The details of the cut joints are described in Table 4.3. All axles have two wheels. The tire-ground contact forces are modeled through Pacejka's magic formula [83]. The semi-trailer suspension and the tractor rear suspension are made up of air springs and dampers, whereas the tractor front suspension is made up of leaf springs and dampers. The joint between the semitrailer and the tractor has been modeled as a spherical joint. Detailed information about the semi-trailer truck model is described in Table 4.4.

\begin{tabular}{ccccc}
\hline ID & Joint type & \multicolumn{2}{c}{ Connected bodies } & Connected points \\
\hline 1 & $\mathrm{R}$ & fl_aux11 & f_axle & $\mathbf{r}_{a}, \mathbf{r}_{b}$ \\
2 & $\mathrm{R}$ & fr_aux9 & f_axle & $\mathbf{r}_{c}, \mathbf{r}_{d}$ \\
3 & $\mathrm{R}$ & fr_aux12 & f_axle & $\mathbf{r}_{e}, \mathbf{r}_{f}$ \\
4 & $\mathrm{R}$ & fr_dir & ct_dir & $\mathbf{r}_{g}, \mathbf{r}_{h}$ \\
5 & $\mathrm{R}$ & fr_aux17 & f_axle_2 & $\mathbf{r}_{i}, \mathbf{r}_{j}$ \\
6 & $\mathrm{~S}$ & tractor_chassis & semitrailer_chassis & $\mathbf{r}_{k}, \mathbf{r}_{l}$ \\
7 & $\mathrm{R}$ & rr_aux26 & r_axle_m1 & $\mathbf{r}_{m}, \mathbf{r}_{n}$ \\
8 & $\mathrm{R}$ & rr_aux30 & r_axle_m2 & $\mathbf{r}_{o}, \mathbf{r}_{p}$ \\
9 & $\mathrm{R}$ & rr_aux34 & r_axle_m3 & $\mathbf{r}_{q}, \mathbf{r}_{s}$ \\
\hline
\end{tabular}

Table 4.3: Cut joints

\subsubsection{Accuracy}

The sedan vehicle and semi-trailer truck have been simulated for $1 \mathrm{~s}$ with four different time-steps to investigate the accuracy. The absolute maximum difference and the norm of the difference in the position array $(\mathbf{z})$ between the presented and original algorithms are gathered in Table 4.5. The size of the position array is 34 for the sedan vehicle and 80 for the semi-trailer truck, corresponding to the number 


\begin{tabular}{lr}
\hline Item & Number \\
\hline Bodies (including auxiliary bodies) & 81 \\
Auxiliary bodies & 34 \\
Joints (including cut joints) & 89 \\
Axles & 5 \\
Dependent relative coordinates $(n)$ & 80 \\
Independent relative coordinates $(f)$ & 39 \\
Driving coordinate & 1 \\
Constraint equations $(m)$ & 43 \\
Eliminated revolute joints & 8 \\
Eliminated spherical joints & 1 \\
DOF & 40 \\
\hline
\end{tabular}

Table 4.4: Element count

of dependent relative coordinates.

\begin{tabular}{cccccc}
\hline & & \multicolumn{4}{c}{ Errors } \\
\cline { 3 - 6 }$h(\mathrm{~ms})$ & Loop count & \multicolumn{2}{c}{ Sedan vehicle } & \multicolumn{2}{c}{ Semi-trailer truck } \\
\cline { 3 - 6 } & & $\max (|\Delta \mathbf{z}|)$ & $\operatorname{norm}(\Delta \mathbf{z})$ & $\max (|\Delta \mathbf{z}|)$ & $\operatorname{norm}(\Delta \mathbf{z})$ \\
\hline 0.1 & 40000 & $4.77 \times 10^{-15}$ & $4.90 \times 10^{-13}$ & $4.21 \times 10^{-15}$ & $6.56 \times 10^{-13}$ \\
0.2 & 20000 & $6.17 \times 10^{-15}$ & $3.18 \times 10^{-13}$ & $1.78 \times 10^{-15}$ & $9.23 \times 10^{-13}$ \\
0.5 & 8000 & $2.90 \times 10^{-15}$ & $2.75 \times 10^{-13}$ & $3.55 \times 10^{-15}$ & $2.85 \times 10^{-13}$ \\
1 & 4000 & $3.67 \times 10^{-15}$ & $2.01 \times 10^{-13}$ & $7.11 \times 10^{-15}$ & $2.48 \times 10^{-13}$ \\
\hline
\end{tabular}

Table 4.5: Error incurred by iterative refinement with different time-steps

In order to visualize these differences, the $X, Y$ and $Z$ translations of the chassis are plotted. The $h=1 \mathrm{~ms}$ responses are compared in Figures 4.5 and 4.6 for the sedan vehicle and semi-trailer truck, respectively. It can be seen that the errors are extremely small for both models. 

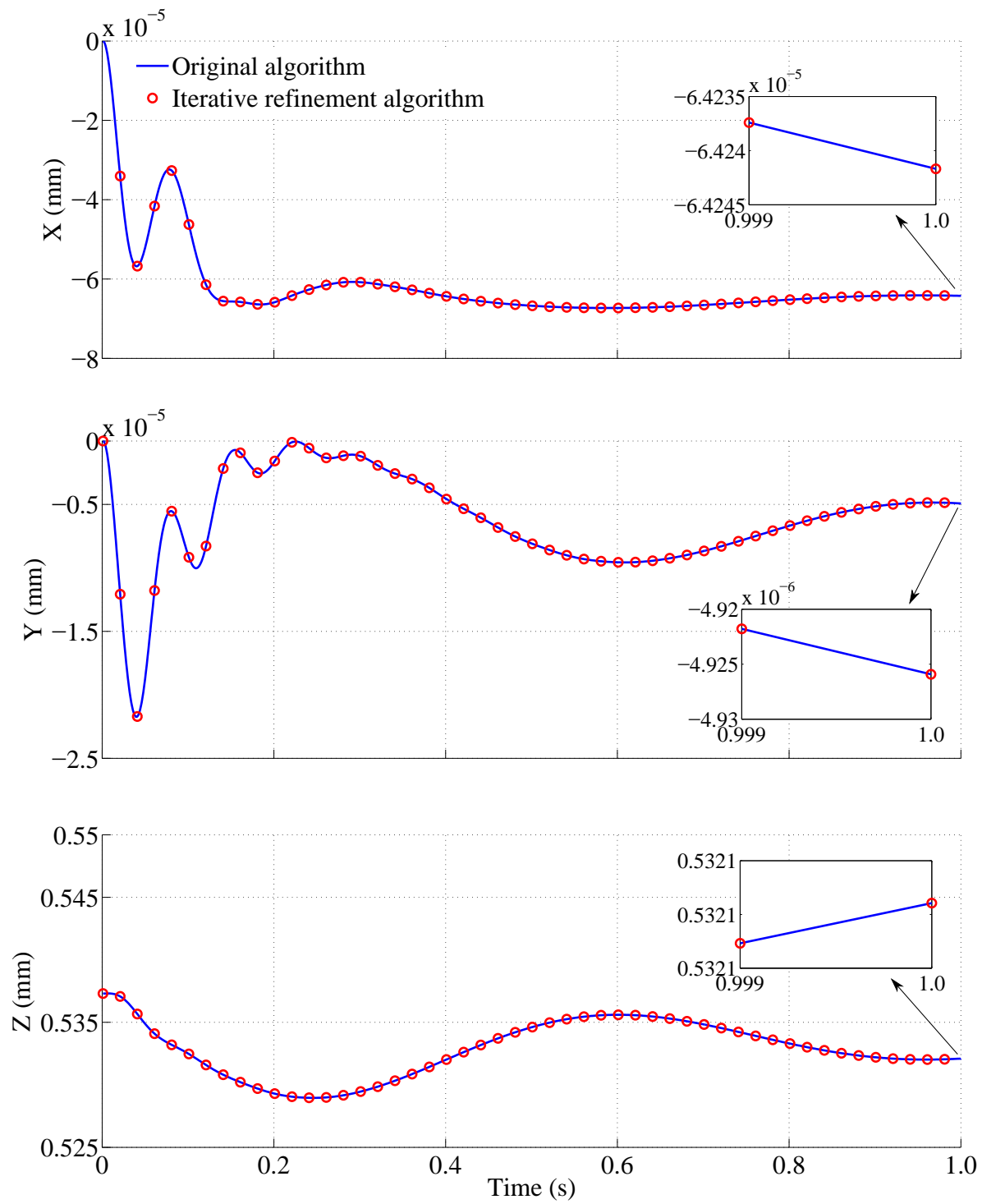

Figure 4.5: $X$-, $Y$ - and $Z$-translations of the sedan vehicle chassis 

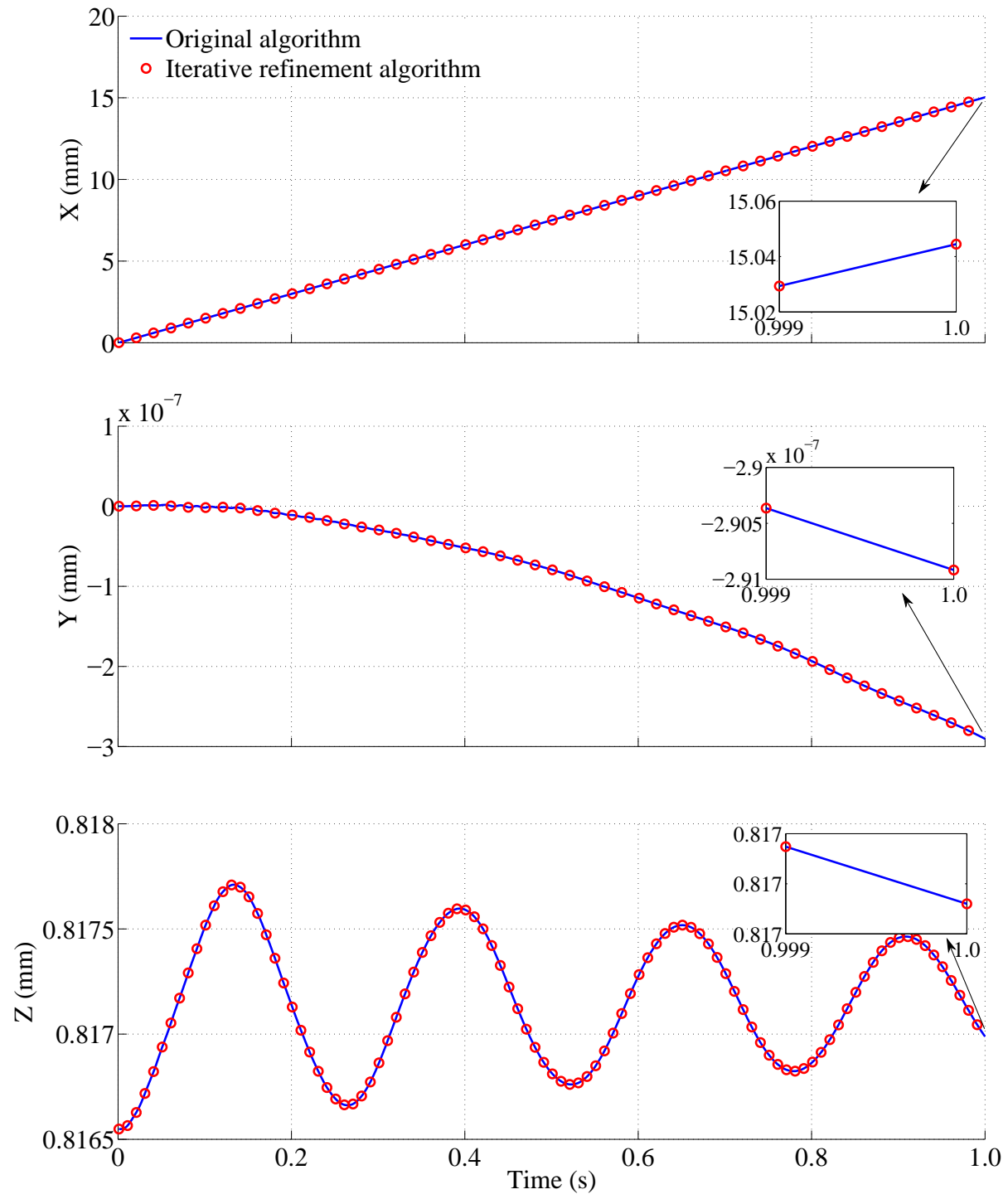

Figure 4.6: $X-, Y$ - and $Z$-translations of the semi-trailer truck chassis 


\subsubsection{Efficiency}

The dynamic simulations have been timed for the presented and original algorithms. All simulations have been run on an Intel Core i7 laptop with a $2.4 \mathrm{GHz} \mathrm{CPU}$ and $8 \mathrm{~GB}$ of RAM. The dynamic maneuvers have been simulated for $10 \mathrm{~s}$ with 1-ms and 0.5 -ms time-steps. The computational burden is mainly in the state vector derivative function, which once again has been implemented in $\mathrm{C} / \mathrm{C}++$ and uses Intel's Math Kernel Library.

Table 4.6 shows the elapsed time of the state vector derivative function within the presented and original algorithms. A fact is that, in the 0.5-ms time-step case, the efficiency gain of $2.7 \%$ and $9.4 \%$ are obtained for the sedan vehicle and semitrailer truck, respectively. The presented iterative refinement algorithm is more efficient that the original 4th-order Runge-Kutta algorithm.

Furthermore, the algorithm is more efficient when used in the semi-trailer truck model. The reason is that the semi-trailer truck model has a larger generalized mass matrix, and avoiding its factorization saves more CPU time.

\begin{tabular}{llcc}
\hline \multirow{2}{*}{$h(\mathrm{~ms})$} & \multirow{2}{*}{ Algorithms } & \multicolumn{2}{c}{ Elapsed time (s) } \\
\cline { 3 - 4 } & & Sedan vehicle & Semi-trailer truck \\
\hline \multirow{2}{*}{0} & Original algorithm & 3.86 & 11.73 \\
& Iterative refinement algorithm & $3.76(\mathbf{2 . 4 \%} \uparrow)$ & $10.67(\mathbf{9 . 0 \%} \uparrow)$ \\
\hline \multirow{2}{*}{0.5} & Original algorithm & 7.66 & 23.45 \\
& Iterative refinement algorithm & $7.45(\mathbf{2 . 7 \%} \uparrow)$ & $21.21(\mathbf{9 . 4 \%} \uparrow)$ \\
\hline
\end{tabular}

Table 4.6: Computational efficiency of iterative refinement technique in $10 \mathrm{~s}$ simulation

Finally, the algorithm is marginally more efficient in the 0.5 -ms time-step case. This is because the initial guess is closer to the exact solution when the smaller time-step is used, and thus few iterations are required, which improves the efficiency. Figure 4.7 contains the iterations of each iterative refinement process during a 1-s simulation for the sedan vehicle and semi-trailer truck. 

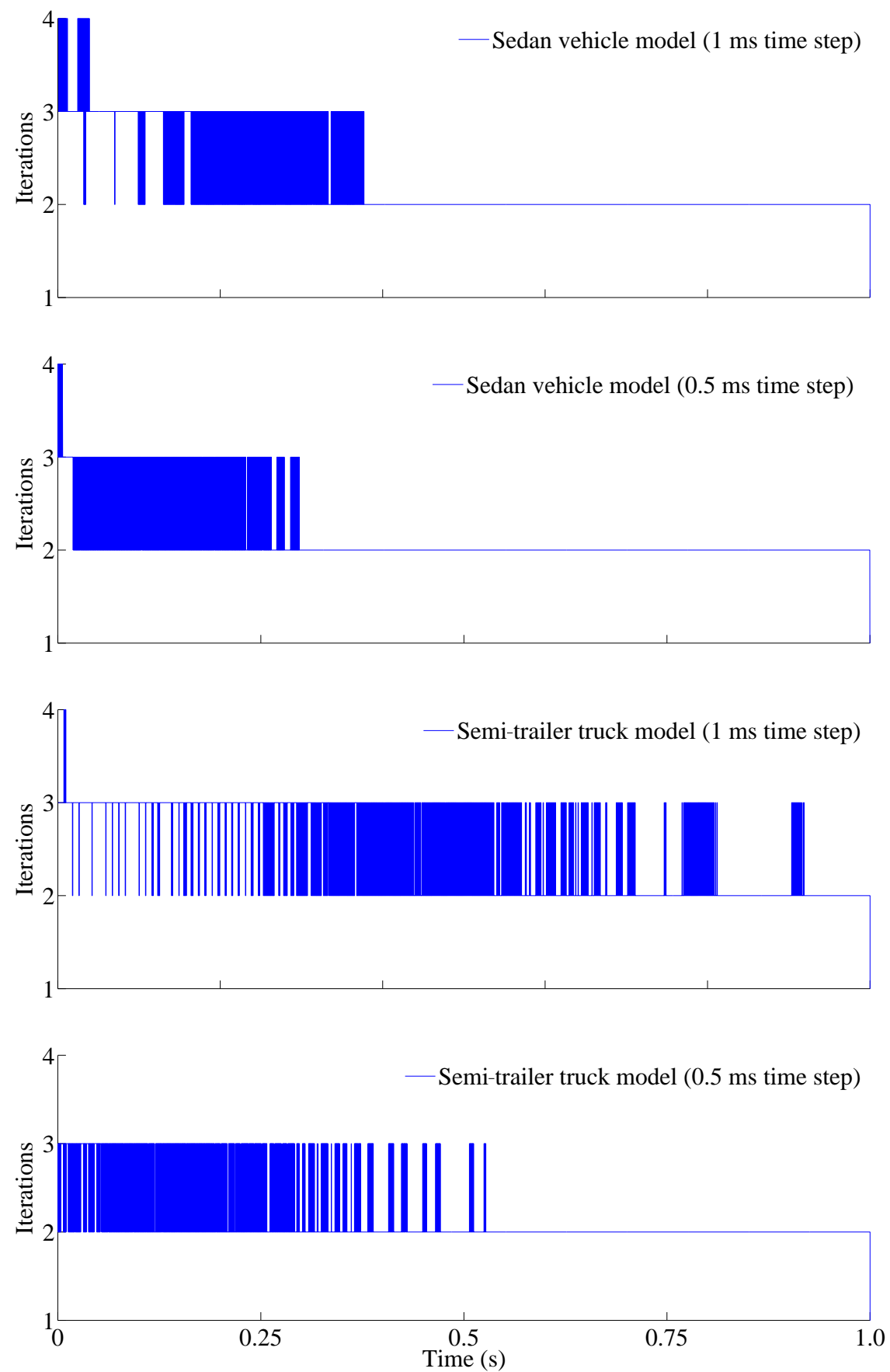

Figure 4.7: Iterations in a $1 \mathrm{~s}$ simulation 


\subsection{Discussion}

In this Chapter an iterative refinement algorithm has been introduced into an explicit 4th-order Runge-Kutta integrator for improved efficiency. In the iterative refinement process, an initial guess close to the exact solution is used and a weighted error norm is introduced as the termination criteria. The computationally expensive generalized mass matrix calculation is avoided. The main advantage of the presented algorithm is the reuse of the generalized mass matrix factorization between neighboring Rugge-Kutta steps.

Afterwards, a medium-size 16-DOF sedan vehicle model and a large-size 40-DOF semi-trailer truck model have been simulated with different time-steps to assess the accuracy and computational efficiency. Results show efficiency gains of $2.7 \%$ and $9.5 \%$ for the sedan vehicle and semi-trailer truck, respectively, along with high accuracy. Further, the algorithm is more efficient for large-size multibody systems. Overall, an iterative refinement algorithm has been presented in the context of efficient time integration schemes for the dynamic simulation of medium-large multibody systems. 


\section{Chapter 5}

\section{Conclusions}

\subsection{Contributions}

A number of efficient formulations for the dynamic simulation of multibody systems are available. They differ from one another in the specific form of the equations of motion, the type of generalized coordinates, the constraint enforcement technique, the time integration scheme, the computer implementation strategy, etc. Even though the theory of rigid-body dynamics has been well-established for centuries, the efficient simulation of multibody systems is still a challenging research topic. The improvement of the computational efficiency, which has been tried for many years, is becoming more and more difficult, especially for those sophisticated formulations [75, 95, 96]. In this Thesis, efficient techniques have been introduced to efficiently implement the double-step semi-recursive formulation for complex vehicle systems. The computational efficiency of three state-of-the-art topological formulations has been fully analyzed based on two medium-size vehicle models. The main contributions can be summarized as follows:

- Three state-of-the-art topological formulations, namely subsystem synthesis formulation, double-step semi-recursive formulation and generalized semirecursive formulation, have been implemented. A 28-DOF rover model and 
a 16-DOF sedan vehicle model have been simulated. Different implicit and explicit integrators have been employed to numerically solve the equations of motion. Simulation results showed that these formulations produce accurate solutions, and the double-step semi-recursive formulation is the most efficient one.

- A rod-removal technique and a second-derivative-based inertia forces (SDIF) approximation method have been introduced into the double-step semirecursive formulation for accurate and efficient rod modeling. The complexity of the system inertia matrix is reduced through the SDIF approximation, where point, linear Lagrange and quadratic Lagrange extrapolations are used. A 15-DOF sedan car model with 12 rods has been fully modeled and simulated to assess the computational efficiency. An efficiency gain of about $10 \%$ was obtained, along with a satisfactory accuracy.

- An iterative refinement technique for the speedup of an explicit 4th-order Runge-Kutta integrator has been presented. The generalized mass matrix and its factorization, which vary very slowly between neighboring Runge-Kutta steps, are avoided in the iterative refinement procedure. An initial guess close to the exact solution is used to reduce the number of iterations. A 16-DOF sedan vehicle and a 40-DOF semi-trailer truck have been fully modeled and simulated to assess the efficiency. Simulation results showed an efficiency gain of $9.4 \%$ in the truck model, along with good accuracy.

Overall, novel contributions to state-of-the-art topological formulations have been presented and backed by the simulation of four medium-large vehicles.

\subsection{Future work}

In this Section, a few suggestions for future development are concisely presented. 
- The computational efficiency and solution stability of the double-step semirecursive formulation based on different integrators, such as Adams-BashfordMoulton (ABM) explicit integrator, could be investigated.

- A deep investigation of how the size and topology of the vehicle system affect the efficiency of topological formulations remains to be carried out.

- It is possible to apply the rod-removal and SDIF approximation methods to other topological formulations such as generalized semi-recursive and subsystem synthesis formulations.

- It should be very interesting to apply the iterative refinement technique to integrators with time-step control such as the ode45 (based on Runge-Kutta method) and ode113 (based on ABM predictor corrector method) Matlab integrators.

- The SDIF approximation and iterative refinement techniques, which have been presented separately, could be implemented simultaneously for those vehicle models heavily depending on rods to further improve the efficiency. 


\section{Bibliography}

[1] F. Aghili and J.-C. Piedbœuf. Simulation of motion of constrained multibody systems based on projection operator. Multibody Syst. Dyn., 10(1):3-16, 2003.

[2] K. S. Anderson. Recursive Derivation of Explicit Equations of Motion for Efficient Dynamic/Control Simulation of Large Multibody Systems. PhD thesis, Stanford University, 1990.

[3] K. S. Anderson. An order $\mathrm{n}$ formulation for the motion simulation of general multi-rigid-body constrained systems. Computers \& Structures, 43(3):565 - 579, 1992.

[4] K. S. Anderson. An order $\mathrm{n}$ formulation for the motion simulation of general multi-rigid-body tree systems. Computers \& structures, 46(3):547-559, 1993.

[5] K. S. Anderson and J. Critchley. Improved 'order-n' performance algorithm for the simulation of constrained multi-rigid-body dynamic systems. Multibody Syst. Dyn., 9(2):185-212, 2003.

[6] K. S. Anderson and M. J. Sadowski. An efficient method for contact/impact problems in multibody systems: Topologies with many loops. In ASME 2003 International Design Engineering Technical Conferences and Computers and Information in Engineering Conference, pages 341-350. American Society of Mechanical Engineers, 2003.

[7] K. S. Anderson and M. J. Sadowski. An efficient method for contact/impact problems in multibody systems: Tree topologies. In ASME 2003 International Design Engineering Technical Conferences and Computers and Information in Engineering Conference, pages 331-340. American Society of Mechanical Engineers, 2003.

[8] J. Angeles. Fundamentals of robotic mechanical systems. Springer, 2002.

[9] M. Arioli, J. W. Demmel, and I. S. Duff. Solving sparse linear systems with sparse backward error. SIAM Journal on Matrix Analysis and Applications, 10(2):165-190, 1989.

[10] M. Arioli and J. Scott. Chebyshev acceleration of iterative refinement. Numerical Algorithms, 66(3):591-608, 2014.

[11] U. M. Ascher, H. Chin, L. R. Petzold, and S. Reich. Stabilization of constrained mechanical systems with DAEs and invariant manifolds. Mech of Struct Mach., 23(2):135-157, 1995. 
[12] A. Avello, J. Jiménez, E. Bayo, and J. García de Jalón. A simple and highly parallelizable method for real-time dynamic simulation based on velocity transformations. Comput. Methods Appl. Mech. Engrg., 107(3):313 - 339, 1993.

[13] D. S. Bae, H. Cho, S. Lee, and W. Moon. Recursive formulas for design sensitivity analysis of mechanical systems. Comput. Methods Appl. Mech. Engrg., 190(29-30):3865 - 3879, 2001.

[14] D. S. Bae, J. M. Han, and J. H. Choi. An implementation method for constrained flexible multibody dynamics using a virtual body and joint. Multibody Syst. Dyn., 4(4):297-315, 2000.

[15] D. S. Bae, J. M. Han, J. H. Choi, and S. M. Yang. A generalized recursive formulation for constrained flexible multibody dynamics. Int. J. Numer. Methods Eng, 50(8):1841-1859, 2001.

[16] D. S. Bae, J. M. Han, and H. H. Yoo. A generalized recursive formulation for constrained mechanical system dynamics. Mech of Struct Mach., 27(3):293-315, 1999.

[17] D. S. Bae and E. J. Haug. A recursive formulation for constrained mechanical system dynamics: Part I. Open loop systems. Mech of Struct Mach., 15(3):359-382, 1987.

[18] D. S. Bae and E. J. Haug. A recursive formulation for constrained mechanical system dynamics: Part II. Closed loop systems. Journal of Structural Mechanics, 15(4):481-506, 1987.

[19] D. S. Bae, J. K. Lee, H. J. Cho, and H. Yae. An explicit integration method for realtime simulation of multibody vehicle models. Comput. Methods Appl. Mech. Engrg., 187(1-2):337 - 350, 2000.

[20] J. M. Banerjee and J. J. McPhee. Graph-theoretic sensitivity analysis of multibody systems. J. Comput. Nonlinear Dynam, 9(4):041009, 2014.

[21] J. Baumgarte. Stabilization of constraints and integrals of motion in dynamical systems. Comput. Methods Appl. Mech. Engrg., 1(1):1 - 16, 1972.

[22] J. Baumgarte. A new method of stabilization for holonomic constraints. J. Appl. Mech, 50(4a):869-870, 1983.

[23] J. Baumgarte and E. Stiefel. Examples of transformations improving the numerical accuracy of the integration of differential equations. In Proceedings of the Conference on the Numerical Solution of Ordinary Differential Equations, pages 207-236. Springer, 1974.

[24] E. Bayo and R. Ledesma. Augmented Lagrangian and mass-orthogonal projection methods for constrained multibody dynamics. Nonlinear Dynamics, 9(1-2):113-130, 1996.

[25] H. J. Bowdler, R. S. Martin, G. Peters, and J. H. Wilkinson. Solution of real and complex systems of linear equations. Numerische Mathematik, 8(3):217-234, 1966.

[26] H. Brauchli. Mass-orthogonal formulation of equations of motion for multibody systems. Zeitschrift für angewandte Mathematik und Physik ZAMP, 42(2):169-182, 
1991.

[27] C. Brezinski and M. R. Zaglia. Extrapolation Methods: Theory and Practice. North Holland, 1991.

[28] A. Callejo and J. García de Jalón. A hybrid direct-automatic differentiation method for the computation of independent sensitivities in multibody systems. Int. J. Numer. Methods Eng, 100(12):933-952, 2014.

[29] A. Callejo and J. García de Jalón. Vehicle suspension identification via algorithmic computation of state and design sensitivities. J. Mech. Des, 137(2):021403, 2015.

[30] A. Callejo, J. García de Jalón, P. Luque, and D. A. Mántaras. Sensitivity-based, multi-objective design of vehicle suspension systems. J. Comput. Nonlinear Dynam, 10(3):031008, 2015.

[31] A. Callejo, Y. Pan, J. L. Ricón, J. Kövecses, and J. García de Jalón. Comparison of semi-recursive and subsystem synthesis algorithms for the efficient simulation of multibody systems. Submitted to J. Comput. Nonlinear Dynam, 2016.

[32] P. I. Davies, N. J. Higham, and F. Tisseur. Analysis of the Cholesky method with iterative refinement for solving the symmetric definite generalized eigenproblem. SIAM Journal on Matrix Analysis and Applications, 23(2):472-493, 2001.

[33] J. Demmel, Y. Hida, W. Kahan, X. S. Li, S. Mukherjee, and E. J. Riedy. Error bounds from extra-precise iterative refinement. ACM Trans. Math. Softw., 32(2):325-351, 2006.

[34] H. Dugoff, P. Fancher, and L. Segel. An analysis of tire traction properties and their influence on vehicle dynamic performance. Technical report, SAE Technical Paper, 1970.

[35] R. Featherstone. The calculation of robot dynamics using articulated-body inertias. The International Journal of Robotics Research, 2(1):13-30, 1983.

[36] R. Featherstone. Robot Dynamics Algorithms. Kluwer, 1987.

[37] A. Fijany and R. Featherstone. A new factorization of the mass matrix for optimal serial and parallel calculation of multibody dynamics. Multibody Syst. Dyn., 29(2):169-187, 2013.

[38] P. Flores and J. Ambrósio. On the contact detection for contact-impact analysis in multibody systems. Multibody Syst. Dyn., 24(1):103-122, 2010.

[39] P. Flores, M. Machado, E. Seabra, and M. T. Silva. A parametric study on the Baumgarte stabilization method for forward dynamics of constrained multibody systems. J. Comput. Nonlinear Dynam, 6(1):011019, 2011.

[40] G. E. Forsythe and C. B. Moler. Computer solution of linear algebraic systems. Prentice-Hall, Englewood Cliffs, N.J., 1967.

[41] F. J. Funes. Simulación eficiente de mecanismos flexibles mediante formulaciones topológicas. PhD thesis, Universidad Politécnica de Madrid, 2015.

[42] F. J. Funes and J. García de Jalón. An efficient dynamic formulation for solving rigid and flexible multibody systems based on semi-recursive method and implicit 
integration. J. Comput. Nonlinear Dynam, 11(5):051001, 2016.

[43] J. García de Jalón, E. Álvarez, F. de Ribera, I. Rodríguez, and F. Funes. A fast and simple semi-recursive formulation for multi-rigid-body systems. In J. Ambrósio, editor, Advances in Computational Multibody Systems, volume 2 of Computational Methods in Applied Sciences, Chapter 1, pages 1-23. Springer Netherlands, 2005.

[44] J. García de Jalón and E. Bayo. Kinematic and Dynamic Simulation of Multibody Systems: The Real-Time Challenge. Springer, 1994.

[45] J. García de Jalón and A. Callejo. A straight methodology to include multibody dynamics in graduate and undergraduate subjects. Mechanism and Machine Theory, 46(2):168 - 182, 2011.

[46] J. García de Jalón, A. Callejo, and A. F. Hidalgo. Efficient solution of Maggi’s equations. J. Comput. Nonlinear Dynam, 7(2):021003, 2012.

[47] J. García de Jalón, J. Unda, A. Avello, and J. M. Jiménez. Dynamic analysis of three-dimensional mechanisms in "natural" coordinates. J. Mech., Trans., and Automation, 109(4):460-465, 1987.

[48] G. H. Golub and R. S. Varga. Chebyshev semi-iterative methods, successive overrelaxation iterative methods, and second order Richardson iterative methods. $\mathrm{Nu}$ merische Mathematik, 3(1):147-156, 1961.

[49] F. González, M. Á. Naya, A. Luaces, and M. González. On the effect of multirate cosimulation techniques in the efficiency and accuracy of multibody system dynamics. Multibody Syst. Dyn., 25(4):461-483, 2011.

[50] M. González, D. Dopico, U. Lugrís, and J. Cuadrado. A benchmarking system for MBS simulation software: Problem standardization and performance measurement. Multibody Syst. Dyn., 16(2):179-190, 2006.

[51] M. H. Gutknecht and S. Röllin. The Chebyshev iteration revisited. Parallel Comput., 28(2):263-283, 2002.

[52] M. H. Gutknecht and Z. Strakos. Accuracy of two three-term and three twoterm recurrences for Krylov space solvers. SIAM Journal on Matrix Analysis and Applications, 22(1):213-229, 2000.

[53] M. Haghshenas-Jaryani and A. Bowling. A new switching strategy for addressing Euler parameters in dynamic modeling and simulation of rigid multibody systems. Multibody Syst. Dyn., 30(2):185-197, 2013.

[54] E. J. Haug, D. Negrut, and M. Lancu. A state-space-based implicit integration algorithm for differential-algebraic equations of multibody dynamics. Journal of Structural Mechanics, 25(3):311-334, 1997.

[55] H. Hemami and F. C. Weimer. Modeling of nonholonomic dynamic systems with applications. J. Appl. Mech, 48(1):177-182, 1981.

[56] A. F. Hidalgo. Simulación en tiempo real de vehículos industriales con modelos multicuerpo de gran complejidad. PhD thesis, Universidad Politécnica de Madrid, 2013. 
[57] A. F. Hidalgo and J. García de Jalón. Real-time dynamic simulations of large road vehicles using dense, sparse, and parallelization techniques. J. Comput. Nonlinear Dynam, 10(3):031005, 2015.

[58] N. Higham. Accuracy and Stability of Numerical Algorithms. Society for Industrial and Applied Mathematics, 2002.

[59] W. W. Hooker. A set of $r$ dynamical attitude equations for an arbitrary n-body satellite having r rotational degrees of freedom. AIAA Journal, 8(7):1205-1207, 1970.

[60] M. Jankowski and H. Woźniakowski. Iterative refinement implies numerical stability. BIT Numerical Mathematics, 17(3):303-311, 1977.

[61] W. Jerkovsky. The structure of multibody dynamic equations. Journal of Guidance, Control and Dynamics, 1(3):173-182, 1978.

[62] M. H. Kaplan. Modern spacecraft dynamics \& control. Wiley, 1976.

[63] W. A. Khan, V. N. Krovi, S. K. Saha, and J. Angeles. Recursive kinematics and inverse dynamics for a planar 3R parallel manipulator. J. Dyn. Sys., Meas., Control, 127(4):529-536, 2004.

[64] W. A. Khan, V. N. Krovi, S. K. Saha, and J. Angeles. Modular and recursive kinematics and dynamics for parallel manipulators. Multibody Syst. Dyn., 14(3):419$455,2005$.

[65] S. S. Kim. A subsystem synthesis method for efficient vehicle multibody dynamics. Multibody Syst. Dyn., 7(2):189-207, 2002.

[66] S. S. Kim and W. Jeong. Subsystem synthesis method with approximate function approach for a real-time multibody vehicle model. Multibody Syst. Dyn., 17(23):141-156, 2007.

[67] S. S. Kim, W. H. Jeong, M. H. Kim, and J. B. Han. Comparative study on multibody vehicle dynamics models based on subsystem synthesis method using Cartesian and joint coordinates. Theoretical and Applied Mechanics Letters, 2(6):063010, 2012.

[68] S. S. Kim and C. H. Lee. A recursive subsystem synthesis method for repeated closed loop structure in multibody dynamics. Journal of Mechanical Science and Technology, 23(4):946-949, 2009.

[69] S. S. Kim and M. Vanderploeg. A general and efficient method for dynamic analysis of mechanical systems using velocity transformations. J. Mech. Des, 108(2):176$182,1986$.

[70] S. S. Kim and J. H. Wang. Subsystem synthesis methods with independent coordinates for real-time multibody dynamics. Journal of Mechanical Science and Technology, 19(1):312-319, 2005.

[71] A. Kurdila, J. G. Papastavridis, and M. P. Kamat. Role of Maggi's equations in computational methods for constrained multibody systems. Journal of Guidance, Control and Dynamics, 13(1):113-120, 1990.

[72] J. Kyparisis. On uniqueness of Kuhn-Tucker multipliers in nonlinear programming. 
Mathematical Programming, 32(2):242-246, 1985.

[73] H. M. Lankarani and P. E. Nikravesh. A contact force model with hysteresis damping for impact analysis of multibody systems. J. Mech. Des, 112(3):369-376, 1990.

[74] H. M. Lankarani and P. E. Nikravesh. Continuous contact force models for impact analysis in multibody systems. Nonlinear Dynamics, 5(2):193-207, 1994.

[75] A. Laulusa and O. A. Bauchau. Review of classical approaches for constraint enforcement in multibody systems. J. Comput. Nonlinear Dynam, 3(1):011004, 2007.

[76] C. Lubich. Extrapolation integrators for constrained multibody systems. IMPACT of Computing in Science and Engineering, 3(3):213 - 234, 1991.

[77] W. A. McClelland and A. L. Klosterman. Nastran for dynamic analysis of vehicle systems. Technical report, SAE Technical Paper, 1974.

[78] C. B. Moler. Iterative refinement in floating point. J. ACM, 14(2):316-321, 1967.

[79] R. D. Monteiro and I. Adler. Interior path following primal-dual algorithms: Part I. Linear programming. Mathematical programming, 44(1-3):27-41, 1989.

[80] D. Negrut, R. Serban, and F. A. Potra. A topology-based approach to exploiting sparsity in multibody dynamics: Joint formulation. Mech of Struct Mach., 25(2):221241, 1997.

[81] M. A. Neto and J. Ambrósio. Stabilization methods for the integration of DAE in the presence of redundant constraints. Multibody Syst. Dyn., 10(1):81-105, 2003.

[82] N. Orlandea and M. Chace. Simulation of a vehicle suspension with the ADAMS computer program. Technical report, SAE Technical Paper, 1977.

[83] H. B. Pacejka. Tyre and Vehicle Dynamics. Elsevier Butterworth-Heinemann, 2012.

[84] R. Pàmies-Vilà, J. M. Font-Llagunes, U. Lugrís, F. J. Alonso, and J. Cuadrado. A computational benchmark for 2D gait analysis problems. In New Trends in Mechanism and Machine Science, pages 689-697. Springer, 2015.

[85] Y. Pan, A. Callejo, J. L. Bueno, R. A. Wehage, and J. García de Jalón. Efficient and accurate modeling of rigid rods. Multibody Syst. Dyn., pages 1-20, 2016.

[86] J. G. Papastavridis. Maggi's equations of motion and the determination of constraint reactions. Journal of Guidance, Control and Dynamics, 13(2):213-220, 1990.

[87] R. Paul. Manipulator Cartesian path control. IEEE Transactions on systems, man, and cybernetics, 9(11):702-711, 1979.

[88] D. L. Peiper. The kinematics of manipulators under computer control. Technical report, DTIC Document, 1968.

[89] M. F. S. Pereira and J. A. Ambrósio. Computational dynamics in multibody systems. Springer Science \& Business Media, 2013.

[90] A. B. K. Rao, S. K. Saha, and P. V. M. Rao. Dynamics modelling of hexaslides using the decoupled natural orthogonal complement matrices. Multibody Syst. Dyn., 15(2):159-180, 2006. 
[91] J. L. Ricón. Implementación de la formulación implícita semi-recursiva de Bae para la simulación en tiempo real de sistemas multicuerpo. Master's thesis, ETSII Technical University of Madrid, 2014.

[92] J. I. Rodríguez, J. M. Jiménez, F. J. Funes, and J. García de Jalón. Recursive and residual algorithms for the efficient numerical integration of multi-body systems. Multibody Syst. Dyn., 11(4):295-320, 2004.

[93] S. K. Saha. Dynamics of serial multibody systems using the decoupled natural orthogonal complement matrices. J. Appl. Mech, 66(4):986-996, 1999.

[94] S. K. Saha and W. Schiehlen. Recursive kinematics and dynamics for closed loop multibody systems. Mech of Struct Mach., 29(2):143-175, 2001.

[95] W. Schiehlen. Multibody system dynamics: roots and perspectives. Multibody Syst. Dyn., 1(2):149-188, 1997.

[96] W. Schiehlen. Research trends in multibody system dynamics. Multibody Syst. Dyn., 18(1):3-13, 2007.

[97] W. Schiehlen. History of benchmark problems in multibody dynamics. In Multibody Dynamics: Computational Methods and Applications, pages 357-368. Springer, 2014.

[98] M. Schulz and H. Brauchli. Simulation, sensitivity analysis and optimization of constrained multibody systems with impacts based on mass-orthogonal projections. In IUTAM Symposium on Optimization of Mechanical Systems, pages 261-268. Springer, 1996.

[99] R. Serban and E. J. Haug. Kinematic and kinetic derivatives in multibody system analysis. Journal of Structural Mechanics, 26(2):145-173, 1998.

[100] R. Serban and E. J. Haug. Globally independent coordinates for real-time vehicle simulation. J. Mech. Des, 122:575-582, 2000.

[101] R. Serban, D. Negrut, E. J. Haug, and F. A. Potra. A topology-based approach for exploiting sparsity in multibody dynamics in Cartesian formulation. Mech of Struct Mach., 25(3):379-396, 1997.

[102] M. Serna, R. Avilés, and J. García de Jalón. Dynamic analysis of plane mechanisms with lower pairs in basic coordinates. Mechanism and Machine Theory, 17(6):397 403, 1982.

[103] D. Singh and V. Goel. Stability of single track vehicles. Vehicle System Dynamics, 4(2-3):116-120, 1975.

[104] R. D. Skeel. Iterative refinement implies numerical stability for Gaussian elimination. Math. Comp., 35:817-832, 1980.

[105] W. Stelzle, A. Kecskeméthy, and M. Hiller. A comparative study of recursive methods. Archive of Applied Mechanics, 66(1):9-19, 1995.

[106] G. W. Stewart. Introduction to Matrix Computations. Academic Press, New York, 1973.

[107] J. Unda, J. García de Jalón, F. Losantos, and R. Enparantza. A comparative study 
on some different formulations of the dynamic equations of constrained mechanical systems. J. Mech., Trans., and Automation, 109(4):466-474, 1987.

[108] R. von Schwerin. Multibody System Simulation, Numerical Methods, Algorithms and Software. Springer, 1999.

[109] G. Wahba. A least squares estimate of satellite attitude. SIAM review, 7(3):409-409, 1965.

[110] M. W. Walker and D. E. Orin. Efficient dynamic computer simulation of robotic mechanisms. J. Dyn. Sys., Meas., Control., 104(3):205-211, 1982.

[111] C. Wampler, K. Buffinton, and J. Shu-hui. Formulation of equations of motion for systems subject to constraints. J. Appl. Mech, 52(2):465-470, 1985.

[112] R. A. Wehage and E. J. Haug. Generalized coordinate partitioning for dimension reduction in analysis of constrained dynamic systems. J. Mech. Des, 104(1):247$255,1982$.

[113] P. Wheeler. Tracked vehicle ride dynamics computer program. Technical report, SAE Technical Paper, 1977.

[114] J. H. Wilkinson. Rounding Errors in Algebraic Processes. Dover Publications, Incorporated, 1994.

[115] J. Wittenburg. Dynamics of Systems of Rigid Bodies. B. G. Teubner Stuttgart, 1977.

[116] J. Yen, E. J. Haug, and F. A. Potra. Numerical method for constrained equations of motion in mechanical systems dynamics. Technical report, Center for Simulation and Design Optimization, University of Iowa, 1990. 